Version OCtober 29, 2018

Preprint typeset using $\mathrm{LAT}_{\mathrm{E}} \mathrm{X}$ style emulateapj v. 08/22/09

\title{
COMET C/2011 W3 (LOVEJOY): ORBIT DETERMINATION, OUTBURSTS, DISINTEGRATION OF NUCLEUS, DUST-TAIL MORPHOLOGY, AND RELATIONSHIP TO NEW CLUSTER OF BRIGHT SUNGRAZERS
}

\author{
Zdenek Sekanina \& Paul W. Chodas \\ Jet Propulsion Laboratory, California Institute of Technology \\ 4800 Oak Grove Drive, Pasadena, CA 91109 \\ Version October 29, 2018
}

\begin{abstract}
We describe the physical and orbital properties of C/2011 W3. After surviving the perihelion passage, the comet was observed to undergo major physical changes. The permanent loss of the nuclear condensation and the formation of a narrow spine tail was observed first at Malargue, Argentina, on December 20 and then systematically at Siding Spring, Australia. The process of disintegration culminated with an outburst (terminal fragmentation event) on December 17.6 UT. The postperihelion tail, observed for $\sim 3$ months, was the product of activity over $<2$ days. Because of the delayed response to the hostile environment in the immediate proximity of the Sun, the nucleus' breakup and crumbling was probably caused by thermal stress due to the penetration of intense heat pulse deep into the nucleus' interior after perihelion. The same mechanism may be responsible for cascading fragmentation of sungrazers at large heliocentric distances. The observed behavior is at odds with the rubble-pile model, since the residual mass of the nucleus after perihelion, estimated at $\sim 10^{12} \mathrm{~g}$ (a sphere $\sim 150-200$ $\mathrm{m}$ across), still possessed significant cohesive strength. The spine tail - the product of the terminal outburst - was a synchronic feature, whose brightest part contained submillimeter-sized dust particles, released at velocities not exceeding $30 \mathrm{~m} \mathrm{~s}^{-1}$. The loss of the nuclear condensation prevented an accurate orbital-period determination by traditional techniques. Since the missing nucleus must have been located on the synchrone, whose orientation and sunward tip have been measured, we compute the astrometric positions of this missing nucleus as the coordinates of the points of intersection of the spine tail's axis with the lines of forced orbital-period variation, derived from the orbital solutions based on high-quality preperihelion astrometry from the ground. The resulting orbit gives $698 \pm 2$ years for the osculating orbital period, which proves that $\mathrm{C} / 2011 \mathrm{~W} 3$ is the first major member of the expected new, 21st-century cluster of bright Kreutz-system sungrazers, whose existence was predicted by these authors in 2007. From the spine tail's evolution, we determine that its measured tip, populated by dust particles 1-2 $\mathrm{mm}$ in diameter, receded antisunward from the computed position of the missing nucleus. The bizarre appearance of the comet's dust tail in images taken only hours after perihelion with the coronagraphs on board the SOHO and STEREO spacecraft is readily understood. The disconnection of the comet's head from the tail released before perihelion and an apparent activity attenuation near perihelion have a common cause - sublimation of all dust at heliocentric distances smaller than about 1.8 solar radii. The tail's brightness is strongly affected by forward scattering of sunlight by dust. From an initially broad range of particle sizes, the grains that were imaged the longest had a radiation-pressure parameter $\beta \simeq 0.6$, diagnostic of submicron-sized silicate grains and consistent with the existence of the dust-free zone around the Sun. The role and place of C/2011 W3 in the hierarchy of the Kreutz system and its genealogy via a 14th century parent suggest that it is indirectly related to the celebrated sungrazer $\mathrm{X} / 1106 \mathrm{C} 1$, which, just as the first-generation parent of C/2011 W3, split from a common predecessor during the previous return to perihelion.

Subject headings: comets: general - comets: individual (comet of A.D. 467, X/1106 C1, comet of 1314, X/1381 V1, C/1843 D1, C/1880 C1, C/1882 R1, C/1887 B1, C/1945 X1, C/1963 R1, C/1965 S1, C/1970 K1, D/1993 F2, C/2011 W3) — methods: data analysis
\end{abstract}

\section{INTRODUCTION}

The Kreutz system of sungrazing comets (e.g., Kreutz 1888, 1891, 1901, Marsden 1967, 1989, 2005, Sekanina 2002a, 2003, Sekanina \& Chodas 2004, 2007, 2008) offers the ultimate example of an advanced phase of cascading fragmentation (Sekanina \& Chodas 2007), a process that was shown to occur throughout the orbit, including the aphelion region (150-200 AU from the Sun). Separation velocities acquired by fragments at each fragmentation

Electronic address: Zdenek.Sekanina@jpl.nasa.gov event generate orbit variations that depend strongly on the breakup's heliocentric distance (Sekanina 2002a). At a single tidally-triggered or tidally-assisted event in the immediate proximity of perihelion (which takes place in the Sun's inner corona), a separation velocity of $1 \mathrm{~m} \mathrm{~s}^{-1}$ causes the two fragments to return to perihelion at times that are typically some 80 years apart. The same separation velocity at a single nontidal splitting event far from the Sun, including the aphelion region, affects the orbital period hardly at all, but, depending on the velocity's direction, can introduce material changes in the 
other orbital elements: up to $\sim \frac{1}{7} R_{\odot}\left(1 R_{\odot}=\right.$ Sun's radius or $0.0046548 \mathrm{AU}$ ) in the perihelion distance (thus allowing some fragments to collide with the Sun's photosphere at the next perihelion passage), up to $\sim 5^{\circ}$ in the longitude of the ascending node and the argument of perihelion, and up to $\sim 1^{\circ}$ in the orbit inclination. Given that the separation velocity can easily reach a few meters per second, the resulting effects on the orbital elements substantially exceed those by the indirect planetary perturbations considered by Marsden (1967).

The observed long-term temporal distribution of the bright sungrazers does indeed show a tendency toward clumping, with the interval between two consecutive clusters averaging about 80 years (e.g., Marsden 1967, Sekanina \& Chodas 2007). In addition, the four major fragments of comet C/1882 R1, the brightest known member of the Kreutz system since the beginning of the 18th century (Kreutz 1888, 1891) were found to have actual orbital periods that increase also with an average step of 80 years, from $\sim 600$ to $\sim 840$ years (Sekanina \& Chodas 2007).

Considering this recurrence cycle, given that the two most recent clusters of bright Kreutz sungrazers peaked in the 1880s and 1960s, noting that the clusters were preceded, two to more than three decades earlier, by precursor sungrazers, and also recognizing that the arrival rate of SOHO Kreutz system comets has been climbing ever since the launch of the spacecraft (Sekanina \& Chodas 2007, Knight et al. 2010), the authors predicted in 2007 that "another cluster of bright sungrazers is expected to arrive in the coming decades, the earliest member possibly just several years from now" (Sekanina \& Chodas 2007).

With T. Lovejoy's discovery of C/2011 W3 (Green 2011), the question has arisen whether this is indeed the first major member of the predicted 21st-century cluster. The answer depends critically on the accurate determination of the comet's orbital period $P$. If $P$ is about 400 years or shorter, the comet should be a fragment of one of the sungrazers reported in the course of the 17th-century or even more recently, and has nothing in common with a new cluster. On the other hand, if the orbital period is substantially longer than 400 years, then $\mathrm{C} / 2011 \mathrm{~W} 3$ should indeed belong to the new cluster.

\section{PREPERIHELION GROUND-BASED OBSERVATIONS}

When discovered on November 27, C/2011 W3 had only $\sim 18$ days to reach perihelion (which occurred on 2011 December 16.0 UT), and there was little hope for an accurate determination of the orbital period from preperihelion data, regardless of their quality. Observing circumstances were unfavorable for ground-based imaging, since the comet's elongation from the Sun was rapidly decreasing, from $50^{\circ}$ at discovery to merely $17^{\circ} .6$ on December 10, when its position was measured from the ground for the last time before perihelion. Still, more than 100 astrometric positions were obtained during this two-week period (Spahr et al. 2011, 2012), the great majority of which was sufficiently accurate and mutually consistent to be used for deriving a high-quality set of elements, except for the orbital period. For example, two sets of elliptical elements computed by Williams (2011a, 2011b) from 91 observations between November 27 and December 8 and from 94 observations between November 27 and
Table 1

Temporal Coverage of the Head of Comet C/2011 W3 BY THE SOHO AND STEREO IMAGing INSTRUMENTS ${ }^{\mathrm{a}}$

\begin{tabular}{|c|c|c|c|c|}
\hline \multirow[b]{2}{*}{ Spacecraft } & \multirow{2}{*}{$\begin{array}{c}\text { Imaging } \\
\text { instru- } \\
\text { ment }\end{array}$} & \multirow{2}{*}{$\begin{array}{l}\text { Spatial } \\
\text { resolu- } \\
\text { tion }^{\mathrm{b}}\end{array}$} & \multicolumn{2}{|c|}{ Coverage: 2011 December (UT) } \\
\hline & & & preperihelion & postperihelion \\
\hline \multirow[t]{2}{*}{$\mathrm{SOHO}$} & $\mathrm{C} 2$ & $11^{\prime \prime} .4$ & $15.75-15.96$ & $16.07-16.22$ \\
\hline & $\mathrm{C} 3$ & $56^{\prime \prime}$ & $14.09-15.90$ & $16.15-18.36$ \\
\hline \multirow[t]{3}{*}{ STEREO-A } & COR1 & $7^{\prime \prime} .5$ & $15.87-15.96$ & $16.09-16.16$ \\
\hline & COR2 & $14^{\prime \prime} .7$ & $15.35-15.92$ & $16.13-16.66$ \\
\hline & HI1 & $70^{\prime \prime}$ & $12.01-14.92$ & $16.65-22.37$ \\
\hline \multirow[t]{3}{*}{ STEREO-B } & COR1 & $7^{\prime \prime} .5$ & $15.88-15.96$ & $16.23-16.45$ \\
\hline & COR2 & $14^{\prime \prime} .7$ & $15.35-15.90$ & $16.38-17.35$ \\
\hline & HI1 & $70^{\prime \prime}$ & $10.81-15.01$ & $17.87-26.95$ \\
\hline All & $\ldots \ldots$ & $\ldots \ldots$ & $10.81-15.96$ & $16.07-26.95$ \\
\hline
\end{tabular}

a Based on the authors' inspection of the images available at these websites: http://sohodata.nascom.nasa.gov/cgi-bin/data_query for SOHO and http://stereo-ssc.nascom.nasa.gov/cg1-bin/1mages for STEREO.

b Per pixel or per two binned pixels, as used.

December 10, gave osculating orbital periods of, respectively, $376 \pm 51$ years (leaving a mean residual of $\pm 0^{\prime \prime} .7$ ) and $680 \pm 64$ years (leaving $\pm 0^{\prime \prime} .8$ ).

\section{SPACEBORNE OBSERVATIONS NEAR PERIHELION PASSAGE}

Around perihelion, from December 11 to 22, the comet was too close to the Sun to obtain any astrometric positions from the ground. The comet was, however, extensively observed with instruments on board several satellites and deep-space probes. Astrometric data were in fact extracted from the comet's images seen in the coronagraphs and other imaging devices on the $\mathrm{SOHO}$ and both STEREO spacecraft (Kracht 2011, 2012, Spahr et al. 2012). The coverage of the comet's motion by the three spacecraft was excellent over this period of time, as is apparent from Table 1. We use the standard abbreviations for the relevant instruments: $\mathrm{C} 2$ and $\mathrm{C} 3$ for the two coronagraphs on the SOHO spacecraft and COR1 for the inner coronagraph, COR2 for the outer coronagraph, and HI1 for the first heliospheric imager on either of the two STEREO spacecraft. Included in Table 1 is the spatial resolution of the instruments, based on the information from Brueckner et al. (1995) for SOHO and from Howard et al. (2008) for STEREO. The astrometric data from the measured images of the comet obtained by these instruments turned out to be so poor that they could not be combined with the ground-based positions, often of a subarcsec accuracy, to derive high-quality orbital solutions. The best among the spaceborne data are the six positions obtained by Kracht (2011) from the COR2 images of STEREO-B between December 16.49 and 16.57 UT, shortly after perihelion, but even they are not accurate enough to be included in the final iteration of the orbit.

The extensive sets of images taken by the various instruments on board the SOHO and STEREO spacecraft prove more useful for examining the comet's dust-tail morphology (Sec. 10) and may also be useful for studying the light curve in the general proximity of perihelion, even though many of the CCD frames show the comet's 


\section{MAJOR CHANGES IN COMET C/2011 W3 (LOVEJOY) ON 2011 DEC. 17-20}

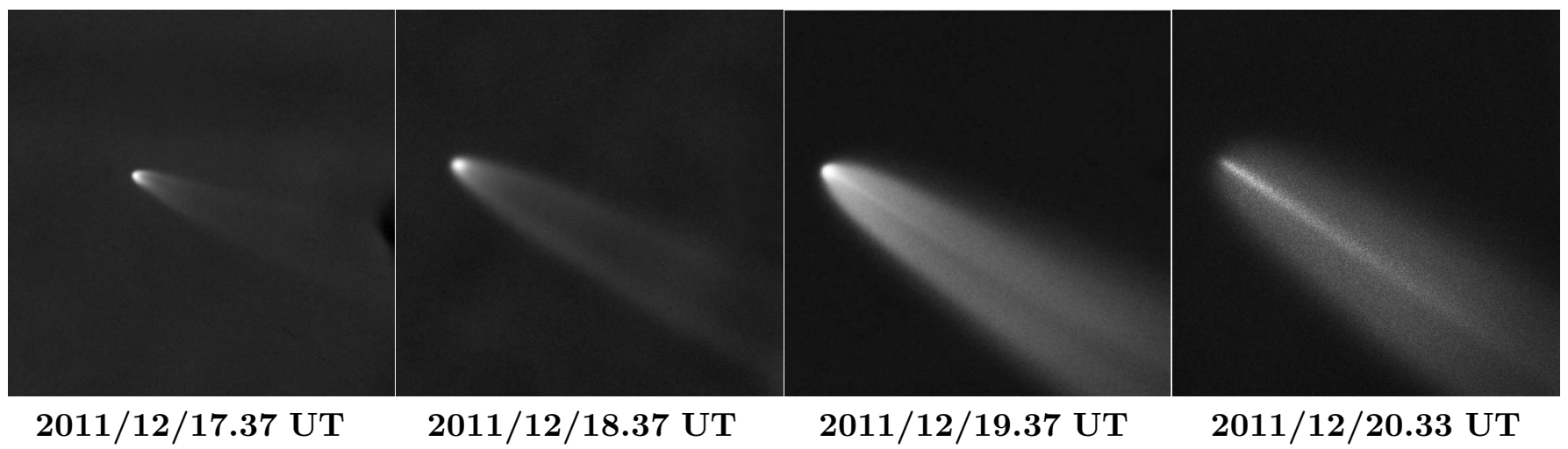

Figure 1. Some of the earliest postperihelion images of Comet C/2011 W3 taken — at the request of J. Cerný — by J. Ebr, M. Prouza, P. Kubánek, and M. Jelínek with the FRAM 30-cm f/10 Schmidt-Cassegrain reflector, a robotic, remotely controled telescope at the Pierre Auger Observatory, Malargue, Argentina. Each frame is approximately $11^{\prime}$ on a side, corresponding to some $430,000 \mathrm{~km}$ in the first image and $375,000 \mathrm{~km}$ in the last image. North is up, east to the left. The comet was $5^{\circ} .8$ from the Sun during the first exposure, $8^{\circ} .5$ during the second, $11^{\circ} .0$ during the third, and $13^{\circ} .3$ during the last one. These images provided the first evidence of the major physical changes in the comet's morphological appearance, which culminated with the sudden, complete loss of the nuclear condensation on December 20. (Image credit: J. Černý, Czech Astronomical Society.)

head saturated. We will not discuss the complex issues of the SOHO and STEREO photometry, but would like to call attention to a few very preliminary findings from our cursory inspection of the images taken with the $\mathrm{C} 2$ and C3 coronagraphs on board SOHO. Most of the inspected images display the saturation artifact known as "blooming," which has for similar integration times been assumed to measure approximately the amount of excess brightness by the number of affected pixels and therefore by the length of the overflow streak. Implementation of this admittedly oversimplified rule does, however, lead to conclusions that are consistent with the results based on firmer, independent evidence addressed later in this paper. It appears that the comet's activity became significantly lower very close to perihelion over a period of several hours. This apparent attenuation is almost certainly a product of intensive sublimation of dust, as examined in some detail in Secs. 10 and 11.

In any case, it seems that the comet's brightness reached a maximum about 0.3 day before perihelion, followed by rapid fading. Up to three subsequent outbursts (some perhaps multiple) may have occurred, peaking at, respectively, $0.4,0.8$, and about $1 \frac{1}{2}$ days after perihelion, or on December 16.4, 16.8, and $\sim 17.5 \mathrm{UT}$. A rigorous study of the light curve should verify these preliminary results.

\section{EARLY POSTPERIHELION OBSERVATIONS FROM GROUND, AND SUDDEN TRANSFORMATION OF COMET'S APPEARANCE}

Although, astonishingly, the comet was imaged in daylight from the ground as early as 1-2 days after perihelion by several observers (Kronk 2011), the frames contained no reference stars to derive the comet's astrometric positions. Among these early postperihelion observations was a set of images taken by a group of Czech observers between December 17.4 and 20.3 UT, who used a robotic, remotely controled telescope, a FRAM 30-cm f/10 Schmidt Cassegrain reflector of the Pierre Auger Observatory at Malargue, Argentina. While no astrometry was possible, comparison of the images obtained on the four days (Fig. 1) shows major changes in the appearance of the comet's head (Černý 2011). From December 17 to 18 the nuclear condensation seemed to have grown and brightened a little, extending in a broad fan in the tailward direction, but otherwise the morphology remained essentially the same. From December 18 to 19 the change was more pronounced; even though the nuclear condensation remained clearly visible, the quasiparabolic contours of the tail became filled with much more material and one can discern a streamer that extended for a few arcminutes nearly along the tail axis. Should it persist and change its orientation predictably with time, such a feature is diagnostic of a sudden, brief outburst of dust from the nucleus (Sec. 5). By itself, an event of this kind may or may not be part of a cataclysmic process that results in the demise of the nucleus. But the stunning change in the comet's appearance between December 19 and 20 strongly suggests that during this episode, portended by the morphological changes during the previous days, the nucleus entirely disintegrated. On December 20 the nuclear condensation completely disappeared (Fig. 1) and the streamer, usually referred to as a spine tail and much longer and more prominent now than the previous day, dominated the comet's head and near-tail region.

On December 20 the comet was imaged at Malargue for the last time. Although the spaceborne observations with the HI1 imagers on both the STEREO-A and STEREO$B$ spacecraft continued past this date (Table 1), their spatial resolution was not sufficient to show the changes detected with clarity in the Malargue images. Fortunately, the observing conditions on the ground were generally improving as the comet's elongation from the Sun steadily continued to increase, and after a three-day gap earth-based monitoring of the comet's head region resumed. 
SPINE TAIL OF COMET C/2011 W3 (LOVEJOY) ON 2011 DEC. 24-2012 JAN. 6

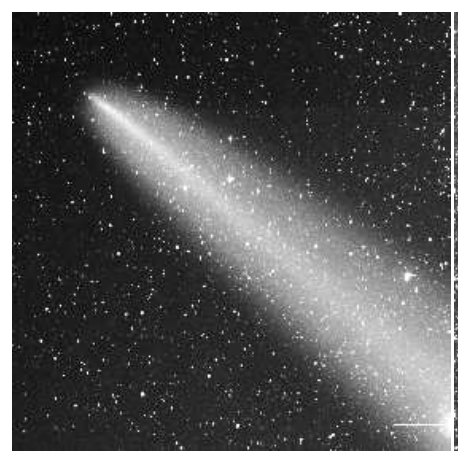

2011/12/24.74 UT

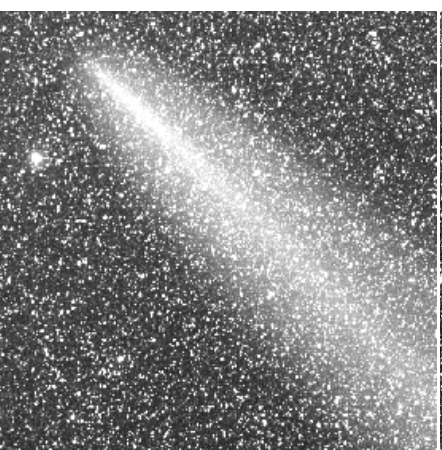

2011/12/28.73 UT

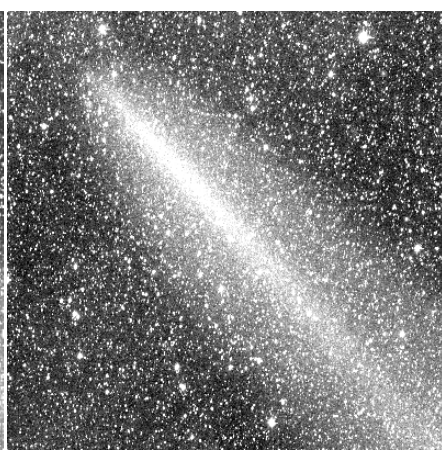

2012/1/1.72 UT

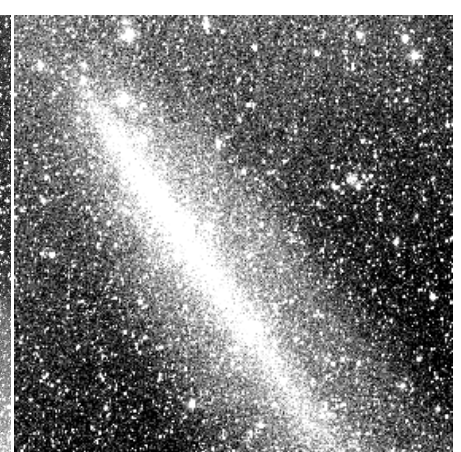

2012/1/6.74 UT

Figure 2. The head and adjoining portion of the spine tail of comet C/2011 W3 taken between 2011 December 24 and 2012 January 6 by R. H. McNaught with the Uppsala 50-cm f/3.5 Schmidt Telescope, Siding Spring Survey. The fields of view are $48^{\prime}$ on a side, corresponding to 1.39 million $\mathrm{km}$ on the first image and 1.04 million $\mathrm{km}$ on the last image. North is up and the east to the left. The Sun is to the upper left, but at an angle to the direction of the tail. Clearly seen is the clockwise rotation and a gradual widening of the spine tail, which is encompassed by a much broader quasi-parabolic envelope. The surface brightness of the spine tail reaches a flat maximum at some distance from the sunward tip; this distance steadily increases with time. The estimated location of the peak surface brightness is populated by dust particles subjected to solar radiation pressure of $\beta_{\text {peak }} \simeq 0.006-0.007$, whose diameter is $400-500 \mu m$ at an assumed bulk density of $0.4 \mathrm{~g} \mathrm{~cm}^{-3}$. [Image credit: R. H. McNaught, Siding Spring Survey (UA/NASA/ANU).]

\section{FOLLOW-UP POSTPERIHELION GROUND-BASED} OBSERVATIONS, AND ORIGIN OF SPINE TAIL

The continuation of the high-resolution monitoring of the comet was very important for finding out whether the loss of the nuclear condensation, so unambiguously documented by the December 20 imaging, became indeed permanent and irreversible. If the nucleus did indeed disappear, there would be no definite point to bisect, and this condition would seem to thwart any attempt at getting accurate postperihelion astrometric data and computing a high-quality set of orbital elements, including a well-determined orbital period.

Starting on December 23, a systematic series of observations of what remained of the comet was begun by McNaught (2012) with the Uppsala 50-cm f/3.5 Schmidt telescope of the Siding Spring Survey in Australia. As seen from the examples displayed in Fig. 2, the comet had nearly the same appearance in all images taken between December 23 and January 18, fully confirming the morphology in the Malargue images of December 20. There was no trace of a nuclear condensation, only a gradually vanishing "hood" at the sunward end of a faint feature with quasi-parabolic contours, on which a much brighter, rectilinear, spine-like tail was superimposed. In the absence of a better choice, McNaught measured what he perceived as possible candidate positions for the tip of the tail at its sunward end, arguably the least objectionable substitute for the missing nucleus.

In spite of this handicap, McNaught's imaging observations provide useful information by allowing one to measure rather precisely (with a $\pm 1^{\circ}$ precision) the systematic clockwise rotation of the spine tail, clearly apparent from Fig. 2. Combined with the measurements of the streamer on the Malargue exposures from December 1920 , the position angle data measured by the authors are listed in Table 2. Each of the measured orientations in column 2 was compared with a set of calculated position angles of synchronic features, loci of dust particles of different sizes subjected to a range of accelerations by solar radiation pressure but released from the nucleus at the same time (e.g., Finson \& Probstein 1968). The distribution of the times of particle release, for which the synchrones' position angles match exactly the measured orientations, have shown a sharp concentration in time, with a random scatter of less than \pm 0.4 day, providing evidence that the spine tail was indeed a product of a major, fairly brief outburst (or a rapid sequence of outbursts) that peaked on December 17.6 $\pm 0.2 \mathrm{UT}$, about 1.6 days after perihelion; the comet was then $0.144 \pm 0.012$ AU from the Sun. (For a preliminary report, see Sekanina 2012.) The predicted variations of the spine tail until mid-February 2012 are displayed in Fig. 3.

The event of December 17.6 (be it single or multiple), which is possibly identical with the last of the three postperihelion outbursts mentioned at the end of Sec. 3, must have begun only a fraction of a day earlier, probably about December 17.2 UT, and led to the disappearance of the nuclear condensation some $2-2 \frac{1}{2}$ days later and to the termination of activity (Sec. 12). Although details of this process are unknown at present, it exhibits characteristics very similar to those of the cataclysmic fragmentation of another sungrazer, comet C/1887 B1 (e.g., Kreutz 1901; Marsden 1967, 2005; Sekanina 1984, 2002a; Sekanina \& Chodas 2004). This general conclusion is supported by the absence, from December 20 on, of any traces of a second tail that would have contained freshly ejected dust; such a tail would have preceded the spine tail some $5^{\circ}$ to $7^{\circ}$ in the clockwise direction.

\section{DEVELOPMENT OF A NOVEL TECHNIQUE FOR ORBIT} DETERMINATION

There have been attempts to determine the orbital elements of C/2011 W3 using some of McNaught's measurements of the tip of the spine tail. The results of one such effort have been published by Williams (2011c). Although he did not list the residuals, he quoted McNaught's remark that "the tip of the 'spine' ... lies pretty much on the front edge of the parabolic hood. It is rather less well defined [on Dec. 24] than on Dec. 23. For this 
Table 2

Orientation of Spine Tail of Comet C/2011 W3

\begin{tabular}{rccc}
\hline \hline & \multicolumn{2}{c}{ Position angle of spine tail } & \\
\cline { 2 - 3 } Date (UT) & measured & residual $^{\mathrm{a}}$ & \\
\hline 2011 Dec. 19.37 & $239^{\circ}$ & +0.15 & Ebserver(s) \\
20.33 & 237 & -0.34 & Ebr al. ${ }^{\mathrm{b}}$ \\
23.75 & 233 & +0.29 & McNaught \\
24.74 & 231 & -0.48 & $"$ \\
26.74 & 229 & -0.12 & $"$ \\
27.74 & 228 & +0.02 & $"$ \\
28.73 & 227 & +0.11 & $"$ \\
29.73 & 225 & -0.82 & $"$ \\
30.73 & 225 & +0.23 & $"$ \\
31.73 & 224 & +0.27 & $"$ \\
1.72 & 223 & +0.29 & $"$ \\
2.73 & 222 & +0.37 & $"$ \\
3.73 & 221 & +0.53 & $"$ \\
6.74 & 214 & -0.17 & $"$ \\
12.45 & 52 & -0.34 & $"$ \\
16.56 & 51 & -0.01 & $"$ \\
18.58 & 52 & +0.06 & $"$ \\
\hline
\end{tabular}

${ }^{\mathrm{a}}$ The difference is: measured minus calculated from a synchronic feature generated by dust outburst on 2011 December 17.6 UT.

${ }^{\mathrm{b}}$ Images reported by J. Černý.

reason I cannot be sure just how closely I am measuring the same point as on Dec. 23" (Williams 2011c). Even though McNaught continued his observations and reductions of the positions of the tip well into January, his results from the post-Dec. 24 images have not been published, nor have they been used by Williams to further update the orbit.

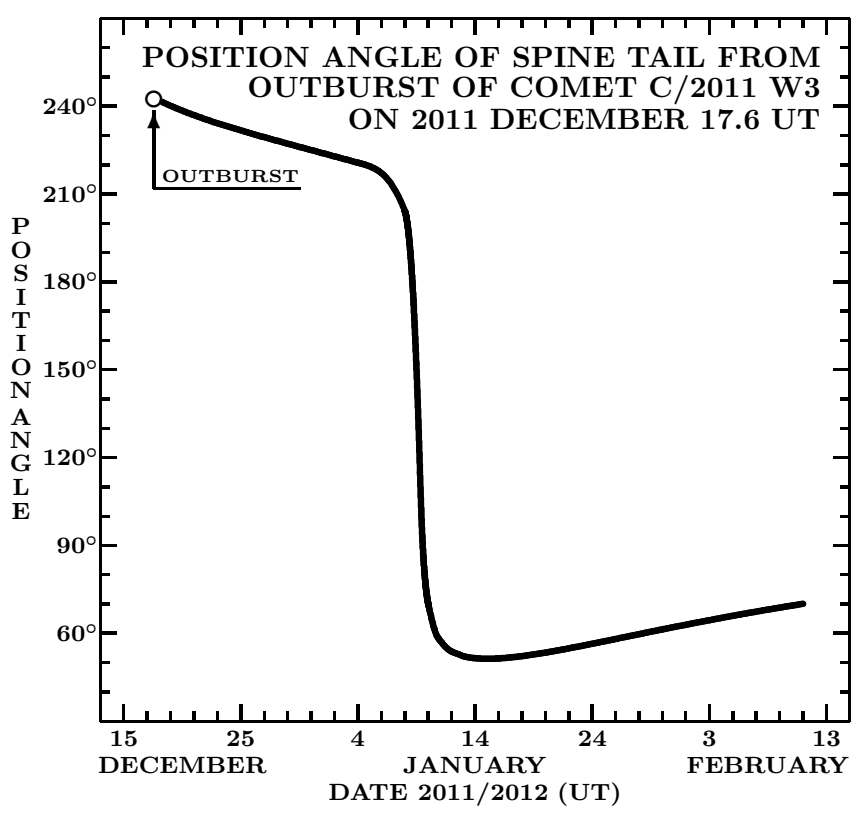

Figure 3. Temporal variations in the position angle (equinox J2000.0) of the spine tail, described as a synchronic feature originating from an outburst on 2011 December 17.6 UT. The steep rate of change between 2012 January 8 and 11 is due to the comet's approach to within $1^{\circ} .5$ of the south celestial pole on January 9 .
Fortunately, McNaught communicated the results of his continuing observations to one of our colleagues, who provided us with the information conveyed (Chesley 2012). The outcome is 46 astrometric positions of the tip of the spine tail, in addition to the three from December 23-24 already published (Spahr et al. 2012). In the following we describe a novel technique that we devised to exploit this set of McNaught's astrometric observations, together with his images of the spine tail. The goal is to extract accurate positions of the missing nucleus and to employ them in our orbit-determination efforts.

Every dust particle released from a comet's nucleus pursues its own orbit in space, which differs from, and is independent of, the comet's subsequent orbit. The orbital deviations are determined by the release time and circumstances as well as the magnitude of solar radiation pressure that the particle has been subjected to after release. Radiation pressure forces the particle to move in a field of reduced effective gravity compared with that acting on the comet. The field intensity is described by the ratio $\beta$ between the acceleration $\gamma_{\mathrm{pr}}$ due to radiation pressure and the Sun's gravitational acceleration $\gamma_{\odot}$. If $G$ is the gravitational constant and $\mathcal{M}_{\odot}$ the mass of the Sun, then, at a heliocentric distance $r, \gamma_{\odot}(r)=G \mathcal{M}_{\odot} / r^{2}$, whereas the acceleration $\gamma_{\mathrm{pr}}(r)$ is given as a product of radiation pressure, $\wp(r)$, and the particle's effective cross sectional area for radiation pressure, $A_{\mathrm{pr}}$, per its mass, $m$. Here $\wp(r)=\mathcal{L}_{\odot} /\left(4 \pi c r^{2}\right)$, with $\mathcal{L}_{\odot}$ being the Sun's total radiation energy emitted per unit time and $c$ the speed of light, while $A_{\mathrm{pr}}$ is the product of the particle's geometrical cross sectional area, $A$, and the efficiency for radiation pressure, $Q_{\mathrm{pr}}$. The ratio $\beta$ can thus be written in the form (e.g., Sekanina et al. 2001)

$$
\beta=\frac{\gamma_{\mathrm{pr}}(r)}{\gamma_{\odot}(r)}=\frac{\mathcal{L}_{\odot} Q_{\mathrm{pr}} A}{4 \pi c G \mathcal{M}_{\odot} m}=\frac{1.15 Q_{\mathrm{pr}}}{\rho x},
$$

where the last expression on the right applies specifically to a spherical grain of a bulk density $\rho$ (in $\left.\mathrm{g} \mathrm{cm}^{-3}\right)$ and diameter $x$ (in $\mu \mathrm{m}$ ). For large particles (tens of microns across and larger) $Q_{\mathrm{pr}} \simeq 1$ (the effective cross section for radiation pressure is nearly identical to the geometrical cross section) and $\beta$ is generally smaller than 0.1 . For particles released from a comet orbiting the Sun in a nearly parabolic path, even this small value of $\beta$ suffices to force the dust move in distinctly hyperbolic orbits concave to the Sun. For strongly absorbing submicron-sized grains $\beta$ can easily exceed unity (a field with acceleration by radiation pressure exceeding gravitational acceleration), and such particles move in hyperbolic orbits convex to the Sun. In the special case of $\beta=1$, particles are subjected to no force and therefore move along straight lines.

As follows from Eq. (1), the ratio $\beta$ is generally independent of heliocentric distance. However, the situation gets a little more complicated when the comet is extremely close to the Sun, as briefly discussed in Sec. 11 .

It is because of the effects of solar radiation pressure that the dust particles of different sizes released during a brief outburst line up in the tail along a synchrone, on which the nucleus is located at $\beta=0$. And because the motions of these particles through the tail are indepen- 
dent of any changes in the comet's motion and behavior after the outburst, including any misfortunes that the nucleus may then incur, the undisturbed positions of the missing nucleus can be recovered from the motion of the synchrone, if one can determine the point at which $\beta=0$. Without an additional constraint the projected position of a disintegrated nucleus is increasingly uncertain, but primarily in one dimension only, along the synchrone.

In practice, a synchrone has a finite breadth for a variety of reasons: a finite duration of the outburst; being the product of a sequence of outbursts; and/or dust particles acquiring lateral velocities upon their release. The general tendency for the synchronic features in the dust tails of comets is to get broader with time, which gradually increases the positional uncertainty across the synchrone.

Over a limited range of fairly low $\beta$ values, the synchrone is a straight line with very high precision (often to better than $\pm 0^{\circ} .1$ in the position angle). If the tip of the spine tail of $\mathrm{C} / 2011 \mathrm{~W} 3$ measured by McNaught is located on the synchrone's axis, the coordinates of the tip and the spine tail's orientation define the synchrone's equation that also fits the undisturbed position of the missing nucleus. If $\left[\alpha_{\text {obs }}(t), \delta_{\text {obs }}(t)\right]$ are the measured equatorial coordinates of the tip of the spine tail and $p(t)$ is the tail's position angle at time $t$, the equatorial coordinates of the missing nucleus, $[\alpha(t), \delta(t)]$, must satisfy a condition

$$
15 \frac{\alpha(t)-\alpha_{\mathrm{obs}}(t)}{\delta(t)-\delta_{\mathrm{obs}}(t)} \cos \delta_{\mathrm{obs}}(t)=\tan p(t),
$$

where $\alpha(t)$ and $\alpha_{\text {obs }}(t)$ are in hours and $\delta(t)$ and $\delta_{\text {obs }}(t)$ in degrees.

To further constrain the equatorial coordinates $\alpha(t)$ and $\delta(t)$, we recall from Sec. 2 that the quality and congruence of the astrometric positions from the preperihelion ground-based observations allowed us to determine accurate sets of orbital elements, except for the osculating period $P$. If these early astrometric data are used to fit orbits with a number of different forced values of the orbital period, the missing nucleus must at any given time be located on this line of orbital-period variation computed for that time. This condition provides a second constraint on the equatorial coordinates $[\alpha, \delta]$ of the missing nucleus, which can readily be found as those of the point of intersection of the line of orbitalperiod variation with the line that satisfies the equation of synchrone in Eq. (2). This result also provides useful information on the orbital period $P$ and determines the separation distance of the measured tip of the spine tail from the missing nucleus and thereby the critical value of $\beta$ for the largest dust grains that are detected at the tail's tip.

Because of the lack of any reliable information on the orbital period at the beginning of this exercise, it is necessary to search for the solution iteratively. In the first approximation, we choose three widely different values of the orbital period, $P_{-1}, P_{0}$, and $P_{1}$, such that $P_{-1}<P_{0}<P_{1}$ and $P_{0}-P_{-1}=P_{1}-P_{0}=\Delta P_{0}$, derive sets of orbital elements from the early astrometry, and for each $t$ compute the corresponding topocentric coordinates $\left[\alpha_{-1}(t), \delta_{-1}(t)\right],\left[\alpha_{0}(t), \delta_{0}(t)\right]$, and $\left[\alpha_{1}(t), \delta_{1}(t)\right]$. We assume that in the given range of orbital periods $P(t)$ both coordinates can be interpolated by fitting, sep- arately for each coordinate, a quadratic law

$$
\begin{aligned}
\alpha(t) & =A(t)+B(t)\left[P(t)-P_{0}\right]+C(t)\left[P(t)-P_{0}\right]^{2}, \\
\delta(t) & =F(t)+G(t)\left[P(t)-P_{0}\right]+H(t)\left[P(t)-P_{0}\right]^{2},
\end{aligned}
$$

where

$$
\begin{aligned}
& A(t)=\alpha_{0}(t), \\
& B(t)=\frac{\alpha_{1}(t)-\alpha_{-1}(t)}{2 \Delta P_{0}}, \\
& C(t)=\frac{\alpha_{-1}(t)+\alpha_{1}(t)-2 \alpha_{0}(t)}{2\left(\Delta P_{0}\right)^{2}},
\end{aligned}
$$

and similarly for $F(t), G(t)$, and $H(t)$. After inserting for $\alpha(t)$ and $\delta(t)$ from Eqs. (3) into Eq. (2) we obtain for $\Delta P(t)=P(t)-P_{0}$ :

$$
\begin{aligned}
& {[H(t)-\zeta(t) C(t)][\Delta P(t)]^{2}+[G(t)-\zeta(t) B(t)] \Delta P(t)} \\
& +F(t)-\delta_{\mathrm{obs}}(t)-\zeta(t)\left[A(t)-\alpha_{\mathrm{obs}}(t)\right]=0
\end{aligned}
$$

where

$$
\zeta(t)=15 \cos \delta_{\text {obs }}(t) \cot p(t) .
$$

One of the $\Delta P(t)$ roots of Eq. (5), which must give $P(t)$ outside the chosen range of $\left\langle P_{-1}, P_{1}\right\rangle$, is ignored. The other root is used to determine the desired topocentric coordinates $[\alpha(t), \delta(t)]$ from Eq. (3). At the end of each iteration the values of $P(t)$ are averaged:

$$
\langle P\rangle=\frac{1}{N} \sum_{i=1}^{N} P(t),
$$

where $N$ is the number of images included in the exercise. It is also verified that scatter of the $P$ values is not excessive (not more than a few percent of $\langle P\rangle$ ) and that their distribution is essentially random. If $\langle P\rangle$ differs substantially from the starting value of $P_{0}$, one should contemplate another iteration by replacing this value of $P_{0}$ with $\langle P\rangle$, tightening the interval $\Delta P_{0}$, and repeating the entire procedure, including the determination of the time of outburst that controls the position angles of the spine tail. The ultimate test is provided by the residuals of the corrected equatorial coordinates of the missing nucleus from the new orbital solution.

\section{COMPUTATIONS AND THE FINAL ORBIT}

To keep the number of necessary iterations of the proposed technique to a minimum, we needed the best possible first-approximation set of orbital elements, including $P$. On the one hand, we learnt that the preperihelion observations alone were inadequate to provide such a set. On the other hand, we found ourselves in an unenviable situation in regard to the sources of postperihelion ground-based data, with no astrometry possible either because of the absence of reference stars in images in which the comet still possessed a nuclear condensation (to bisect in a measuring machine) or because of the loss of the condensation (when reference stars were plentiful). Under these circumstances, we decided to use some of the six positions measured by Kracht (2011) in the STEREOB COR2 images taken about $\frac{1}{2}$ day after perihelion (Sec. 3 ). Combined with the high-quality ground-based positions obtained before perihelion, they offered a solution 


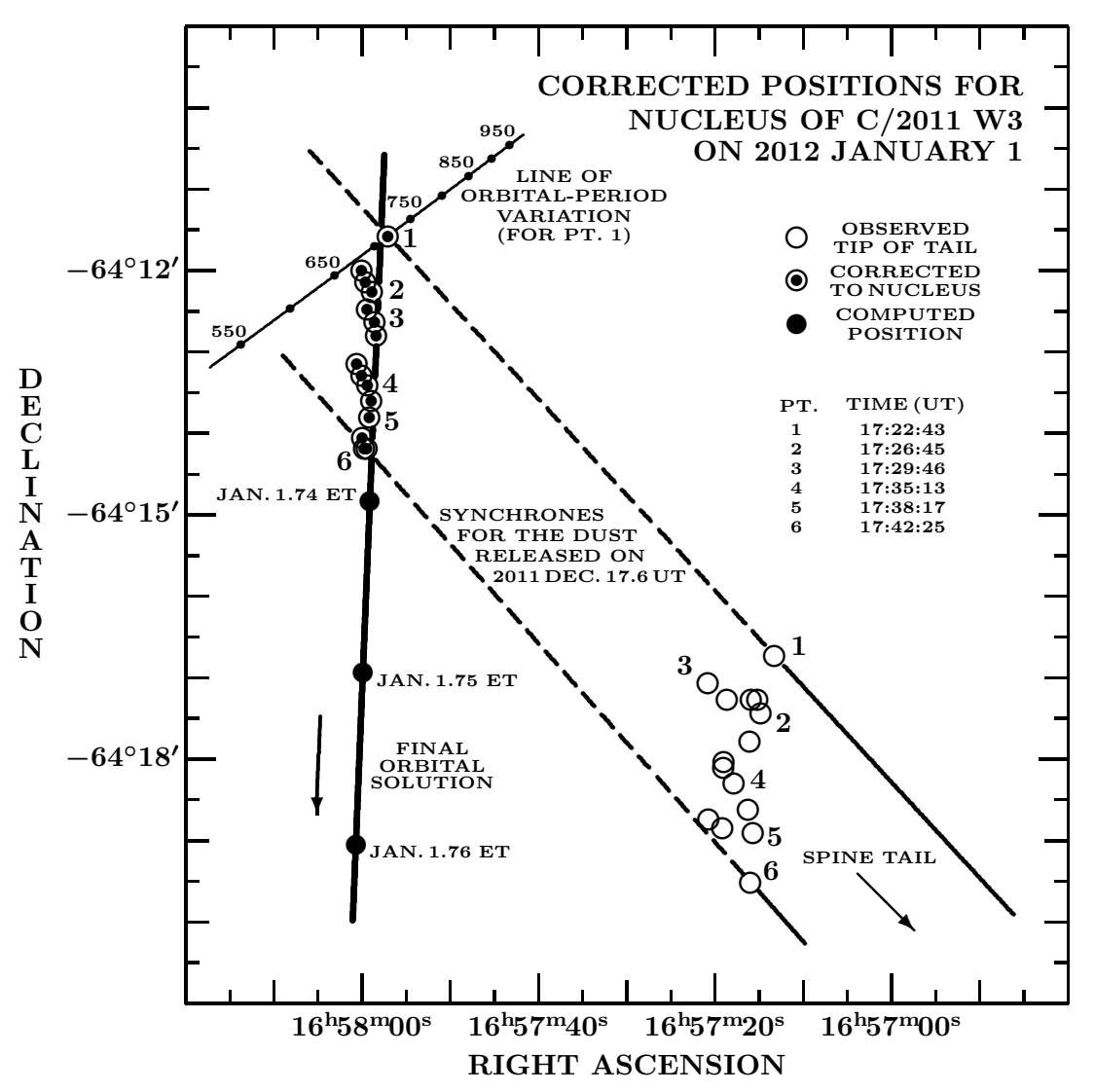

Figure 4. Schematic illustration of the novel technique developed for determining the corrected topocentric positions of the missing nucleus of Comet C/2011 W3 (equinox J2000.0). This example refers to the 15 astrometric positions of the tip of the spine tail measured by R. H. McNaught on 2012 January 1 between 17:22:43 and 17:42:25 UT (open circles). Plotted for the first (Point 1) and the last (Point 6) positions is the spine tail's orientation (medium-thick lines) as a synchrone that refers to the dust outburst on December 17.6 UT. Projected in the direction opposite the direction of the spine tail (broken lines shown again only for Points 1 and 6 ), each of these lines intersects the corresponding line of orbital-period variation, which consists of the predicted topocentric positions of the missing nucleus calculated from the preperihelion high-quality observations as a function of the forced orbital period $P$. Drawn only for the time of Point 1 , this line of orbital-period variation is shown as a thin line calibrated with a number of $P$ values (in years). The points of intersection, plotted as circled dots, are the reconstructed positions of the missing nucleus. We note that the line of orbital-period variation for Point 1 intersects the synchrone at a value of $P$ slightly exceeding 700 years. The heavy line is the resulting orbital solution with the ephemeris positions at three times, plotted by filled circles. The positions of the spine tail's measured tips are seen to be several arcminutes away from the comet's orbit; these distances illustrate the very large magnitude of the introduced errors, if the tip measurements are not corrected.

acceptable for our purpose, with a maximum residual of $9^{\prime \prime}$, and yielded an osculating period of $785 \pm 14$ years.

Based on this finding, we used the same sample of observations to compute three sets of orbital elements by successively forcing the orbital period to 600,800 , and 1000 years. At this point we applied the technique described in Sec. 6 to McNaught's 46 astrometric observations made between 2011 December 23 and 2012 January 6 , and from Eq. (3) we obtained the first set of predicted positions for the missing nucleus. The gist of the procedure is depicted in Fig. 4 on an example of 15 positional measurements obtained by McNaught on 2012 January 1. The average orbital period resulting from this exercise was equal to $\langle P\rangle=709 \pm 6$ years. We got ready for a second iteration by inspecting the used positional data in the orbital run. Having found a solution that is much closer to the true orbital period of the comet, we were no longer critically dependent on the postperihelion data points from STEREO-B. They were not used in the second iteration, because the preperihelion astrometric observations alone allowed computer runs with new forced values of the orbital period. For the nominal run, we chose, in accordance with the developed procedure, $P_{0}=709$ years, and the resulting new set of orbital elements was used to correct the derived position angles of the spine tail. We narrowed down the interval of $\Delta P$ to $\sim 5 \sigma$ of $\langle P\rangle$, or 30 years (i.e., $P_{-1}=679$ years and $P_{1}=739$ years $)$, and proceeded with the second iteration of the missing nucleus' positions. Comparing the results of the two iteration cycles, we noticed that in 38 out of the 46 observations the agreement in the derived positions of the missing nucleus was $6^{\prime \prime}$ or better in either coordinate, and that in the two worst matches the differences amounted to $20^{\prime \prime}$ and $10^{\prime \prime}$, respectively. We felt that another iteration was unnecessary because it would only lead to changes in the subarcsecond range. Consequently, the iterative process was at this point terminated.

For the times of McNaught's exposures, the resulting topocentric positions of the missing nucleus from the final iteration are listed in columns 2 and 3 of Table 3 . Included also are the derived positions from the last three observations that McNaught made on January 12, 16, and 18 , although they are too inaccurate to be used in 
Table 3

Derived Topocentric Positions of the Missing Nucleus of Comet C/2011 W3 and Related Spine-Tail Data

\begin{tabular}{|c|c|c|c|c|c|c|c|}
\hline \multirow{2}{*}{$\begin{array}{c}\text { Observation time } \\
t(\mathrm{UT})\end{array}$} & \multicolumn{2}{|c|}{ Equatorial coordinates } & \multirow{2}{*}{$\begin{array}{l}\text { Distance of } \\
\text { tail's tip from } \\
\text { nucleus, } \ell_{\text {tip }}\end{array}$} & \multirow{2}{*}{$\begin{array}{c}\text { Acceleration } \\
\text { parameter, } \\
\beta_{\text {tip }} \\
\end{array}$} & \multirow{2}{*}{$\begin{array}{c}\text { Particle } \\
\text { diameter, } \\
x_{\text {tip }}(\mathrm{mm}) \\
\end{array}$} & \multicolumn{2}{|c|}{ Residuals $^{\mathrm{a}}$} \\
\hline & R.A.(2000) & Decl.(2000) & & & & R.A. & Decl. \\
\hline 2011 Dec. 23.75224 & $16^{\mathrm{h}}{ }^{\mathrm{m}} 9_{21}^{\mathrm{s}} \cdot 69$ & $-39^{\circ} 25^{\prime} 32^{\prime \prime} .8$ & 1.03 & 0.00198 & 1.45 & +3.5 & -4.8 \\
\hline 24.74267 & 165818.93 & -414414.7 & 1.40 & 0.00202 & 1.42 & $(+4.4$ & $-6.1)$ \\
\hline 24.74785 & 165818.54 & -414457.0 & 1.07 & 0.00154 & 1.87 & +3.2 & -3.8 \\
\hline 26.73669 & 165650.13 & -464058.2 & 1.20 & 0.00107 & 2.69 & +0.7 & +1.6 \\
\hline 26.73853 & 165650.14 & $-4641 \quad 16.6$ & 0.73 & 0.00065 & 4.42 & +1.3 & +0.4 \\
\hline 26.74041 & 165650.32 & -464138.6 & 1.19 & 0.00106 & 2.71 & +3.8 & -4.0 \\
\hline 27.74339 & 165623.95 & -492034.6 & 3.04 & 0.00221 & 1.30 & +0.6 & +1.6 \\
\hline 27.74468 & 165624.05 & -49 2049.1 & 2.54 & 0.00185 & 1.55 & +1.8 & -0.3 \\
\hline 28.73164 & 165610.10 & -520348.9 & 2.75 & 0.00166 & 1.73 & $(-4.2$ & $+8.6)$ \\
\hline 29.73101 & 165609.99 & -545552.1 & 2.89 & 0.00147 & 1.96 & $(-5.7$ & $+9.4)$ \\
\hline 30.72663 & 165626.64 & -575419.8 & 2.79 & 0.00122 & 2.36 & +3.8 & -2.5 \\
\hline 31.73210 & 165700.27 & -610101.5 & 4.89 & 0.00184 & 1.56 & -3.1 & +4.1 \\
\hline 31.73270 & 165659.89 & $\begin{array}{lll}-61 & 01 & 05.6\end{array}$ & 5.15 & 0.00194 & 1.48 & $(-6.0$ & $+6.8)$ \\
\hline 31.73329 & 165700.37 & $\begin{array}{lll}-61 & 01 & 15.4\end{array}$ & 5.40 & 0.00203 & 1.42 & -2.7 & +3.7 \\
\hline 31.73387 & 165700.26 & $\begin{array}{lll}-61 & 01 & 21.0\end{array}$ & 4.55 & 0.00171 & 1.68 & -3.7 & +4.8 \\
\hline 31.73447 & 165701.36 & $\begin{array}{lll}-61 & 01 & 35.1\end{array}$ & 4.70 & 0.00177 & 1.62 & +4.0 & -2.5 \\
\hline 2012 Jan. 1.72411 & 165757.11 & -641134.9 & 7.01 & 0.00231 & 1.24 & $(-8.8$ & $+7.0)$ \\
\hline 1.72505 & 165800.08 & -641159.9 & 7.18 & 0.00236 & 1.22 & $(+10.2$ & $-6.9)$ \\
\hline 1.72599 & 165759.62 & -641208.4 & 6.99 & 0.00230 & 1.25 & $(+6.7$ & $-4.3)$ \\
\hline 1.72691 & 165758.90 & -641215.5 & 7.05 & 0.00232 & 1.24 & +1.5 & -0.6 \\
\hline 1.72786 & 165759.43 & -641228.8 & 6.52 & 0.00215 & 1.34 & +4.5 & -2.7 \\
\hline 1.72901 & 165758.57 & -641237.8 & 6.04 & 0.00199 & 1.44 & -1.7 & +1.9 \\
\hline 1.72995 & 165758.44 & $\begin{array}{llll}-64 & 12 & 47.9\end{array}$ & 6.78 & 0.00223 & 1.29 & -3.0 & +2.8 \\
\hline 1.73089 & 165800.61 & -64 1309.1 & 6.65 & 0.00219 & 1.31 & $(+10.7$ & $-7.3)$ \\
\hline 1.73184 & 165800.05 & -641317.3 & 6.56 & 0.00216 & 1.33 & $(+6.6$ & $-4.3)$ \\
\hline 1.73279 & 165759.39 & -641325.0 & 6.64 & 0.00218 & 1.32 & +1.8 & -0.8 \\
\hline 1.73395 & 165758.98 & -641336.3 & 6.83 & 0.00225 & 1.28 & -1.4 & +1.6 \\
\hline 1.73492 & 165759.18 & -641348.4 & 6.95 & 0.00229 & 1.26 & -0.6 & +0.9 \\
\hline 1.73587 & 165800.01 & -641403.2 & 6.53 & 0.00215 & 1.34 & +4.4 & -2.7 \\
\hline 1.73682 & 165759.46 & -641411.3 & 6.19 & 0.00204 & 1.41 & +0.3 & +0.4 \\
\hline 1.73779 & 165759.75 & $\begin{array}{lll}-64 & 1422.9\end{array}$ & 7.00 & 0.00230 & 1.25 & +1.7 & +0.3 \\
\hline 2.72541 & 165928.62 & -672952.8 & 6.39 & 0.00186 & 1.55 & -4.9 & +2.7 \\
\hline 2.72634 & 165928.49 & -673003.3 & 5.40 & 0.00157 & 1.83 & $(-6.2$ & $+3.5)$ \\
\hline 2.72731 & 165929.53 & -673018.0 & 6.16 & 0.00179 & 1.61 & -0.9 & +0.6 \\
\hline 2.72826 & 165927.73 & -673023.7 & 7.11 & 0.00207 & 1.39 & $(-11.8$ & $+6.4)$ \\
\hline 2.72920 & 165927.78 & -673034.8 & 6.75 & 0.00196 & 1.47 & $(-12.2$ & $+6.7)$ \\
\hline 3.73303 & 170145.81 & -705427.0 & 7.45 & 0.00194 & 1.48 & $(-20.7$ & $+6.5)$ \\
\hline 3.73398 & 170145.84 & -705438.6 & 7.76 & 0.00202 & 1.42 & $(-21.3$ & $+6.7)$ \\
\hline 3.73493 & 170145.82 & -705450.1 & 7.94 & 0.00206 & 1.40 & $(-22.2$ & $+6.9)$ \\
\hline 3.73587 & 170144.23 & -705458.8 & 7.81 & 0.00203 & 1.42 & $(-30.8$ & $+9.9)$ \\
\hline 3.73682 & 170149.66 & -705519.4 & 7.59 & 0.00197 & 1.46 & -5.0 & +1.1 \\
\hline 6.73909 & 172241.59 & -812411.4 & 8.60 & 0.00169 & 1.70 & $(-28.4$ & $-5.0)$ \\
\hline 6.74001 & 172254.11 & $\begin{array}{lll}-81 & 2421.1\end{array}$ & 8.65 & 0.00170 & 1.69 & -2.3 & -3.0 \\
\hline 6.74095 & 172239.36 & -812445.4 & 8.04 & 0.00158 & 1.82 & $(-37.4$ & $-15.3)$ \\
\hline 6.74189 & 172246.40 & -812452.5 & 8.51 & 0.00168 & 1.71 & $(-23.7$ & $-10.4)$ \\
\hline 6.74282 & 172258.62 & -812455.5 & 8.94 & 0.00176 & 1.63 & +1.6 & -1.6 \\
\hline 12.44813 & 41023.94 & $-7838 \quad 18.4$ & 23.29 & 0.00340 & 0.85 & $(-626.6$ & $-531.2)^{\mathrm{b}}$ \\
\hline 16.56300 & 42930.00 & -655851.9 & 12.67 & 0.00171 & 1.68 & $(-98.0$ & $-125.8)$ \\
\hline 18.58108 & 43231.89 & -603549.1 & 10.75 & 0.00143 & 2.01 & $(-104.9$ & $-153.3)$ \\
\hline
\end{tabular}

a The difference is: the position derived from measurement minus the position computed from the final orbital solution; the residual in R.A. includes the factor $\cos ($ Decl.); the positions whose residuals are parenthesized have not been used in the solution.

$\mathrm{b}$ The starting position of the tail's tip appears to be grossly in error.

the orbital solution. In column 4 the table lists the angular distance $\ell_{\text {tip }}$ of the measured tip of the spine tail from the nucleus' predicted position. From an ephemeris of the December 17.6 synchrone this angular distance is converted in column 5 into the radiation-pressure parameter $\beta_{\text {tip }}$, which in the given range varies practically linearly with the angular distance and which, according to Eq. (1), is diagnostic of the diameter $x_{\text {tip }}$ of the dust particles that were detected by McNaught at the tip of the spine tail. These are the largest particles in the observed tail. For an assumed particle bulk density of $0.4 \mathrm{~g}$ $\mathrm{cm}^{-3}$ (e.g., Richardson et al. 2007), this particle diameter is given in column 6. Remarkably, Table 3 and Fig. 5 show that the values of $\beta_{\text {tip }}$ and $x_{\text {tip }}$ did not vary systematically during the nearly four weeks of McNaught's observation, averaging, respectively, $0.00191 \pm 0.00042$ and $1.6 \pm 0.5 \mathrm{~mm}$. On the other hand, the angular distance $\ell_{\text {tip }}$ was increasing nonuniformly, but steadily, with time, 
resembling the rate of recession of a separated companion fragment from the nucleus of its parent comet.

The tabulated topocentric positions of the missing nucleus were at this point incorporated into the input file for the final orbit determination run. It should be remembered that McNaught's intention was to measure positions of the candidates of the tail's tip, not positions of extreme points on the tail's axis, which would be ideal for our orbit determination efforts. On the days on which he took more than one image, it is likely that the tail's tip candidates differed from image to image, as is abundantly clear from his comments to Williams (2011c). And as the spine tail steadily broadened, the chance that any measured tip candidate happened to be in fact located very close to the axis became increasingly remote. One should therefore expect that for only some of the derived astrometric positions of the missing nucleus will the residuals from the final orbit be acceptable for the orbit determination run. Also expected is that the quality of these positions should deteriorate with the spine tail's growing breadth and therefore with time.

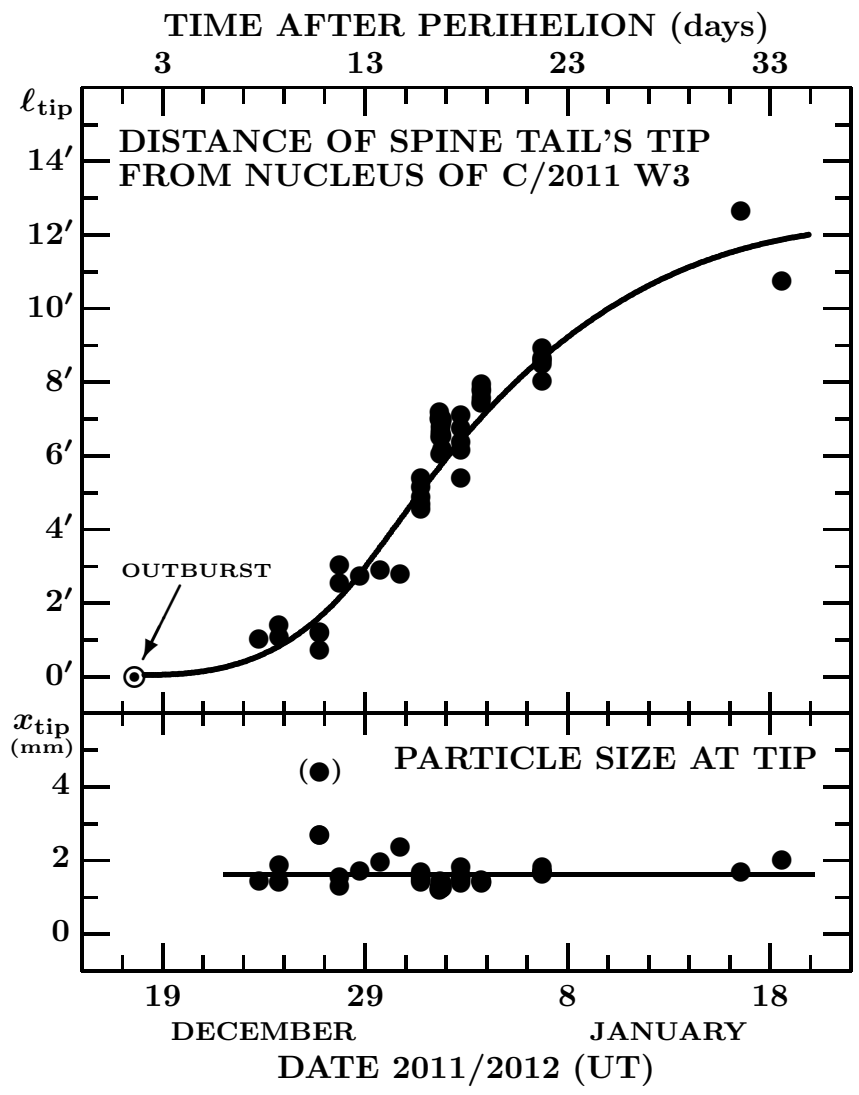

Figure 5. Angular distance $\ell_{\text {tip }}$ of the spine tail's tip, measured by McNaught in his images of C/2011 W3, taken with the Uppsala Schmidt Telescope between December 23 and January 18, as a function of time (top); and the derived diameter of dust particles situated at the tip (bottom). The distance increases with time, whereas the particle dimensions remain the same.

The final orbital solution, which includes the relativistic effect, is presented in Table 4 . It is based on a total of 123 observations between 2011 November 27 and 2012 January 6, all ground-based, which left in either coordi- nate a residual not exceeding $5^{\prime \prime}$. Of these, 96 are preperihelion (from November 27 to December 10) and 27 are postperihelion. All postperihelion entries come from Table 3. This means that the position of the missing nucleus was successfully recovered from nearly 60 percent of McNaught's positions of the tail's tip from images he took between Decmeber 23 and January 6. Given the odds our approach was facing, we consider this number to be quite satisfactory. As expected, the three positions between January 12 and 18 turned out to offer unacceptable residuals, and the starting position from January 12 was clearly incorrect. The residuals of all the positions of the missing nucleus are listed in the last two columns of Table 3 , with the rejected positions parenthesized. The residuals from the preperihelion observations are excellent, showing no systematic trends whatsoever, and largely in the subarcsecond range. The two most significant contributions, with images in both cases obtained robotically, were from the Pierre Auger Observatory, Malargue, Argentina (code I47, 42 accepted positions) and from the Remote Astronomical Society Observatory, Mayhill, New Mexico (code E03, 21 accepted observations); combined they account for nearly two-thirds of all used preperihelion positional data.

Out of a total of 46 positions available to us from astrometric measurements of the images taken on board the SOHO and STEREO spacecraft, none satisfied our adopted cutoff of $\pm 5^{\prime \prime}$ for the residuals. As expected from our discussion in Sec. 3, the least discordant were again the measurements by Kracht (2011) from the six COR2-B images of Dec. 16, which left residuals of up to $27^{\prime \prime}$ in right ascension and up to $15^{\prime \prime}$ in declination. The 24 positions from the STEREO-B images between Dec. 10 and 14 showed surprisingly small residuals in declination, of up to $12^{\prime \prime}$, but strongly systematic, entirely unacceptable residuals of up to $60^{\prime \prime}$ in right ascension. The 8 data points from the SOHO images on Dec. 14 had the opposite problem; 5 of them had residuals less than $10^{\prime \prime}$ in right ascension, but all were off by $40^{\prime \prime}$ to $87^{\prime \prime}$ in declination. The least satisfactory was a set of 8 positions from STEREO-A (listed by Spahr et al. 2012), which had residuals scattered wildly from $-94^{\prime \prime}$ to $+97^{\prime \prime}$ in right ascension and from $-78^{\prime \prime}$ to $+4^{\prime \prime}$ in declination.

From the orbital period in Table 4 we conclude that comet $\mathrm{C} / 2011 \mathrm{~W} 3$ is the first major member of the new, 21st-century cluster of bright Kreutz sungrazers whose existence was predicted by the authors of this paper in 2007 (Sec. 1). The comet cannot be the return of a fragment of any of the sungrazing comets observed since the 17th century, contrary to speculations based on preliminary sets of orbital elements. The perihelion distance agrees closely with the perihelion distances of the bright sungrazers C/1843 D1, C/1880 C1, and to a lesser degree $\mathrm{C} / 1963 \mathrm{R} 1$, but all three angular elements have values unlike any of the known bright members of the system. ${ }^{1}$

1 As a matter of historic curiosity, it should be pointed out that the first orbit for the sungrazer C/1945 X1, calculated by Cunningham $(1946 \mathrm{a}, 1946 \mathrm{~b})$, was in fact somewhat similar to the orbit of $\mathrm{C} / 2011 \mathrm{~W} 3\left(\omega=50^{\circ} .9, \Omega=322^{\circ} .3, i=137^{\circ} .0, q=0.0063\right.$ AU), but it was derived from only crudely determined positions of the comet. The photographic plates with the comet's images were properly measured and reduced only in 1952 (Marsden 1967) and none of Marsden's (1989) resulting orbits shows any obvious resemblance to that of $\mathrm{C} / 2011 \mathrm{~W} 3$. 
Table 4

Final Set of Orbital Elements Adopted for Comet C/2011 W3 And Integration One Revolution Back in Time (EQ. J2000.0)

\begin{tabular}{lcc}
\hline \hline Orbital element & Current apparition & Previous return $^{\mathrm{a}}$ \\
\hline Epoch of osculation $(\mathrm{ET})$ & 2011 Dec. 25.0 & 1329 Jan. 6.0 \\
Time of perihelion passage, $t_{\pi}(\mathrm{ET})$ & 2011 Dec. $16.011810 \pm 0.000040$ & $1329 \mathrm{Jan} .4 .9336$ \\
Argument of perihelion, $\omega$ & $53^{\circ} .5103 \pm 0^{\circ} .0020$ & $52^{\circ} .815$ \\
Longitude of ascending node, $\Omega$ & $326^{\circ} .3694 \pm 0^{\circ} .0027$ & $325^{\circ} .276$ \\
Orbital inclination, $i$ & $134^{\circ} .3559 \pm 0^{\circ} .0012$ & $133^{\circ} .811$ \\
Perihelion distance, $q(\mathrm{AU})$ & $0.00555381 \pm 0.00000007$ & 0.0059198 \\
Orbital eccentricity, $e$ & $0.99992942 \pm 0.00000014$ & 0.9999237 \\
Osculating orbital period, $P(\mathrm{yr})$ & $698 \pm 2$ & 684 \\
\hline
\end{tabular}

${ }^{\mathrm{a}}$ Dates in the Julian calendar.

This suggests that there may exist yet another subcategory of bright sungrazers that has never been considered in the evolutionary models of the Kreutz system. Table 4 also shows the results of integrating the orbit back one revolution about the Sun, to the early 14 th century, suggesting the presence of fairly minor perturbations. The related issues and implications of the comet's orbit are addressed in Sec. 13.

\section{PARTICLE VELOCITIES IN SPINE TAIL AND ITS ANOMALOUS BRIGHTNESS PROFILE}

Besides the clearly apparent clockwise rotation of the spine tail, both the Malargue images in Fig. 1 and the Siding Spring images in Fig. 2 show a quasi-parabolic envelope, in which the spine tail is immersed and which, based on its approximate axial orientation, may have originated from the previous outburst that peaked on about December 16.8 UT or 0.8 day after perihelion (Sec. 3 ). While the spine tail dominates the images brightnesswise, the previous outburst was apparently more violent, as suggested by a much greater breadth of the envelope and, consequently, much higher ejection velocities of the released dust. Indeed, if the width of the spine tail and the envelope are both due entirely to lateral velocities of the particles at the time of release, these velocities come out to be typically up to $20-30 \mathrm{~m} \mathrm{~s}^{-1}$ for the spine tail, but up to at least $150 \mathrm{~m} \mathrm{~s}^{-1}$ for the envelope. Whereas the latter velocities are fairly typical of microscopic dust ejected due to an interaction with outflowing gas, the former are so unusually low that they suggest that the entire residual mass of the nucleus simply collapsed.

One may question whether the episode that led to the formation of the spine tail can at all be called an outburst. It may be more appropriate to refer to it as a cataclysmic fragmentation event or terminal collapse or complete disintegration, but we continue to use all these terms interchangeably even after we address the physical nature of the process in Sec. 12.

The distribution of surface brightness along the spine tail, which displays a broad maximum at some distance from the sunward tip in all McNaught's images, is positively peculiar. The gradual rise in the nearhead brightness with increasing distance from the nucleus is anomalous in that it contradicts a typical power law for the particle-size distribution of dust comets. In a low-ejection-velocity approximation, the FinsonProbstein (1968) approach provides the following expression for the variation of the surface brightness $\mathcal{I}$ as a function of the radiation-pressure acceleration parameter $\beta$ along a synchronic feature observed at a time $t_{\mathrm{obs}}$ and made up of dust particles released from the nucleus at a time $t_{\mathrm{obs}}-\tau$ :

$$
\mathcal{I}(\beta) \propto \frac{p \phi(\alpha)}{r^{2}} \frac{\beta^{k-4}}{|\Im(\xi, \eta ; \beta, \tau)|},
$$

where $p$ is the geometric albedo of the particles, $\phi(\alpha)$ their phase function at a phase angle $\alpha$ [normalized to $\left.\phi\left(0^{\circ}\right)=1\right], x^{-k} d x$ is the differential distribution function of particle diameters $x, r$ is the heliocentric distance, and $\Im$ is a Jacobian that converts the radial and transverse rectangular coordinates, $\xi, \eta$, into $\beta$ and $\tau$

$$
\Im(\xi, \eta ; \beta, \tau)=\left|\begin{array}{ll}
\frac{\partial \xi}{\partial \beta} & \frac{\partial \xi}{\partial \tau} \\
\frac{\partial \eta}{\partial \beta} & \frac{\partial \eta}{\partial \tau}
\end{array}\right| .
$$

At a geocentric distance $\Delta$, the coordinates $\xi$ and $\eta$ are projected onto the tangent plane of the sky at the comet's nucleus and reckoned from it, $\xi$ pointing away from the Sun and $\eta$ being normal to it in either direction, as only the absolute value of $\Im$ is used in Eq. (8).

We have already mentioned that at small values of $\beta$ (say, $\beta<0.1$ ), the population of particles released at a time $t_{\mathrm{obs}}-\tau$ and accelerated at a rate $\beta$ is located at an angular distance $\ell$ from the nucleus, which is proportional to $\beta$,

$$
\ell(\beta)=K \beta,
$$

where $K$ is a constant. Similarly, for small values of $\beta$, $\partial \xi / \partial \beta \propto \beta \cdot \Delta$ and $\partial \eta / \partial \beta \propto \beta \cdot \Delta$, while $\partial \xi / \partial \tau \propto \Delta$ and $\partial \eta / \partial \tau \propto \Delta$, so that for such large particles

$$
\Im(\xi, \eta ; \beta, \tau) \propto \beta \cdot \Delta^{2}
$$

and

$$
\mathcal{I}(\ell) \propto \frac{p \phi(\alpha)}{r^{2} \Delta^{2}} \ell^{k-5}
$$

Since the exponent $k$ for the size-distribution function of cometary dust is typically in the range of 3.5 to 4.0 (e.g., Sitko et al. 2011), the surface brightness along the spine tail of $\mathrm{C} / 2011 \mathrm{~W} 3$ is expected to drop with increasing distance $\ell$ from the nucleus with some power of $\ell$, usually between $\ell^{-1.5}$ and $\ell^{-1}$. From Eq. (12) it is obvious that the condition $d(\log \mathcal{I}) / d(\log \ell) \gg 0$ in the part of the 


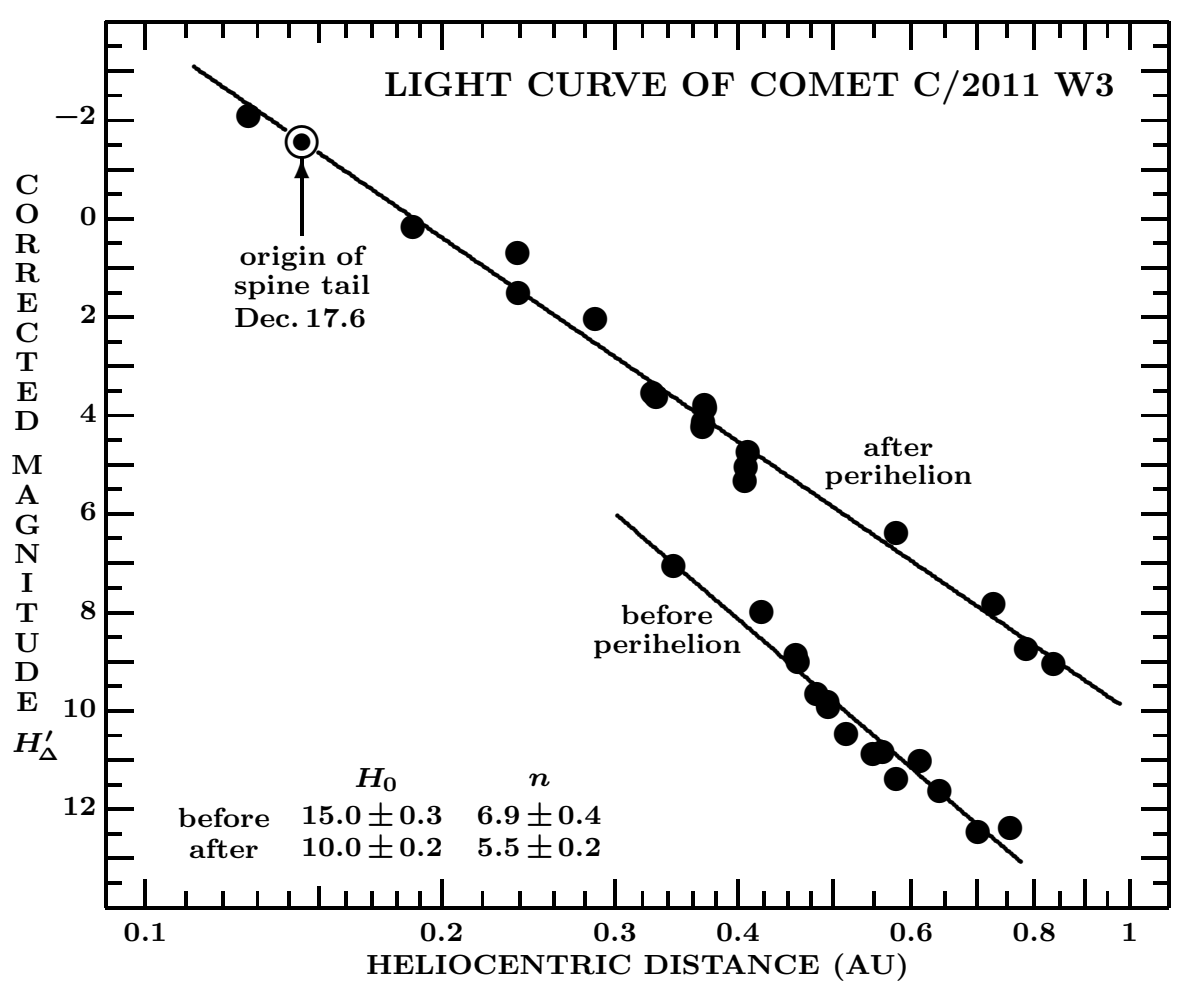

Figure 6. Preliminary light curve of comet C/2011 W3 based on visual and CCD total brightness estimates made from the ground. The magnitudes plotted have been normalized to 1 AU from earth with the inverse square power law and to a zero phase angle with the "compound" Henyey-Greenstein law, as modified by Marcus. Personal and instrumental effects have been corrected to the limited degree possible. The comet is shown to have been much brighter after perihelion than before it.

spine tail between the tip and the peak surface brightness implies a condition

$$
k \gg 5 \text {. }
$$

Such an extremely steep size distribution function can only be explained by crumbling of the largest particles (say, $x>1 \mathrm{~cm}$ ) into progressively smaller ones. Crude visual inspection of McNaught's spine-tail images suggests that the surface brightness attains the peak near $\beta_{\text {peak }} \simeq 0.006-0.007$, or at particle diameters of $\sim 400$ to $500 \mu \mathrm{m}$, independent of the observation time. It is therefore possible that the fragmentation process essentially terminated once fragments of large debris were reduced to sizes of $\sim 100 \mu \mathrm{m}$.

\section{LIGHT CURVE, TAIL BRIGHTNESS, AND TOTAL MASS ESTIMATE FOR RELEASED DUST}

It is hoped that eventually a detailed light curve will be available for comet $\mathrm{C} / 2011$ W3. At this time, however, only fragmentary information has been published. With the brightness data collected primarily from Spahr et al. (2011, 2012) and Green (2012a, 2012b), Figure 6 presents a preliminary light curve, a plot against the heliocentric distance of the magnitude, $H_{\Delta}^{\prime}$, normalized to $1 \mathrm{AU}$ from Earth with the inverse square power law of the geocentric distance and to a zero phase angle with the "compound" Henyey-Greenstein law, as modified for cometary dust by Marcus (2007). The plotted magnitudes were all identified by the observers as total, either visual or CCD, and were corrected by us for personal and instrumental effects to the limited degree possible and referred to the visual photometric system of an average unaided eye. The phase-effect correction is important especially for the postperihelion observations, for which the phase angle was varying between $90^{\circ}$ and $130^{\circ}$ and the effects of forward scattering of sunlight were significant.

The comet was much brighter after perihelion than before, unquestionably due to its cataclysmic fragmentation (Secs. 5 and 8). Figure 6 does not explicitly show the outbursts that are mentioned in Sec. 3, but this is hardly significant given the poor coverage of the light curve near the Sun and low accuracy of the data. From Fig. 6 it appears that to a first approximation, one can fit both branches of the light curve with the traditional power law $r^{-n}$, with $n$ equal to $6.9 \pm 0.4$ before perihelion and $5.5 \pm 0.2$ after perihelion, and with the intrinsic magnitude (at $1 \mathrm{AU}$ from both the Sun and earth), $H_{0}$, equal $15.0 \pm 0.3$ before perihelion and $10.0 \pm 0.2$ after perihelion. It is possible that without the outbursts the postperihelion light curve would have gotten flattened at heliocentric distances below $\sim 0.2 \mathrm{AU}$.

The primary purpose for incorporating the light curve into the scope of this paper has been our intention to use it for estimating the total mass of dust involved in the fragmentation process culminating on December 17.6 UT and therefore the residual mass of the nucleus at breakup. If the visual brightness at the time were due entirely to scattering of sunlight by dust released from the disintegrating nucleus into the atmosphere, one could readily compute the total cross sectional area of the particulate material, once a value for the geometric albedo of the optically thin cloud is adopted. In practice, unfortunately, sodium atoms radiating in the doublet near $5900 \AA$ are a 
Table 5

Upper Limit on Total Amount of Dust from Fragmentation of Comet C/2011 W3

\begin{tabular}{|c|c|c|c|c|c|c|}
\hline \multirow{2}{*}{$\begin{array}{c}\text { Upper limit on } \\
\text { particle size }^{\mathrm{a}}, \\
x_{\max }(\mathrm{cm}) \\
\end{array}$} & \multicolumn{3}{|c|}{ Total mass of dust, $\left(\mathcal{M}_{\text {dust }}\right)_{\max }(\mathrm{g})$} & \multicolumn{3}{|c|}{ Effective diameter, $\mathcal{D}_{\max }(\mathrm{km})$} \\
\hline & $k=3.5$ & $k=3.8$ & $k=4.1$ & $k=3.5$ & $k=3.8$ & $k=4.1$ \\
\hline 10 & $0.9 \times 10^{14}$ & $5.5 \times 10^{12}$ & $7.7 \times 10^{11}$ & 0.76 & 0.30 & 0.15 \\
\hline $10^{2}$ & $3.0 \times 10^{14}$ & $9.0 \times 10^{12}$ & $8.2 \times 10^{11}$ & 1.12 & 0.35 & 0.16 \\
\hline $10^{3}$ & $9.3 \times 10^{14}$ & $14.5 \times 10^{12}$ & $8.6 \times 10^{11}$ & 1.65 & 0.41 & 0.16 \\
\hline
\end{tabular}

${ }^{\mathrm{a}}$ Lower limit to particle diameters is always $x_{\mathrm{min}}=0.1 \mu \mathrm{m}$, the bulk density $\rho_{\mathrm{dust}}=0.4 \mathrm{~g} \mathrm{~cm}^{-3}$.

strong contributor to the visual brightness of SOHO sungrazing comets (Biesecker et al. 2002), so that the light curve provides us with only an upper limit to the cross sectional area of the dust particles and indirectly to the mass of the disintegrated nucleus.

An independent approach to determining the amount of released dust is to estimate the total visual brightness of the comet's tail at a time sufficiently long after the fragmentation process has been completed. Because the tail includes all dust released by the comet since its perihelion passage, the result in this case is an estimate of the mass of the nucleus at, or very shortly after, perihelion. This result would represent a lower limit: not only would all sodium have sublimated away and ionized long before such an observation, but by this time the surface brightness of some of the released debris would have already dropped below the detection threshold of visual observers.

The immediate goal of either of the two approaches is the determination of $H_{\text {eff }}$, the effective visual magnitude of the estimated light scattered by the debris, normalized to the heliocentric and geocentric distances of $1 \mathrm{AU}$ with an inverse square power law, to a zero phase angle with the Marcus (2007) formula, and to the photometric system of an average unaided eye. Expressed in $\mathrm{km}^{2}$, the effective cross sectional area of the dust in the cloud, $X_{\mathrm{dust}}$, is given by an equation

$$
X_{\text {dust }}=\frac{1.54}{p} \times 10^{6-0.4 H_{\text {eff }}},
$$

where $p$ is the geometric albedo of the dust, for which we use a value of 0.04 (e.g., Lamy et al. 2009). For the Sun's visual magnitude we adopt in Eq. (14) a value of -26.65 (corresponding to the $V$ magnitude of -26.75 ). There is no explicit phase function involved because $H_{\text {eff }}$ has already been corrected for this effect.

To be able to estimate the total mass of dust that corresponds to the derived cross sectional area, one has to assume the following: (i) an upper limit on diameters of the particles, $x_{\max }$, (ii) the lower limit, $x_{\min }$, (iii) their size distribution function between $x_{\min }$ and $x_{\max }$, which, as in the preceding section, is given by a power law $x^{-k} d x$, and (iv) their average bulk density, $\rho_{\text {dust }}$. The relation between the total mass of dust, $\mathcal{M}_{\text {dust }}$, and the cross sectional area, $X_{\text {dust }}$, is given by

$$
\mathcal{M}_{\text {dust }}=\frac{2(k-3)}{3(4-k)} \Theta \rho_{\text {dust }} X_{\text {dust }} x_{\min }^{k-3} x_{\max }^{4-k}
$$

when $3<k<4$, and by

$$
\mathcal{M}_{\text {dust }}=\frac{2(k-3)}{3(k-4)} \Theta \rho_{\text {dust }} X_{\text {dust }} x_{\text {min }}
$$

when $k>4$. The coefficient $\Theta$, usually near unity, is given by an expression

$$
\Theta=\frac{1-\left(x_{\min } / x_{\max }\right)^{|k-4|}}{1-\left(x_{\min } / x_{\max }\right)^{k-3}} .
$$

We use $\rho_{\text {dust }}=0.4 \mathrm{~g} \mathrm{~cm}^{-3}$ (e.g., Richardson et al. 2007), which is probably a good estimate for larger grains, but must be an underestimate for microscopic dust. As for $x_{\text {min }}$, particles smaller than $0.1 \mu \mathrm{m}$ in diameter were detected in comets, e.g., in the atmosphere of $1 \mathrm{P} /$ Halley with the mass spectrometers on board the Giotto and VEGA spacecraft, but their contribution to the total mass of dust was found to be low (Utterback \& Kissel 1990, 1995; Sagdeev et al. 1989), so that adopting a lower limit of $x_{\min }=0.1 \mu \mathrm{m}$ seems to be justified.

The exponent $k$ of the power law and the upper limit $x_{\max }$ were in this exercise varied. In conformity with the findings for a large number of comets, $k$ has been constrained to a range of 3.5 to 4.1 (e.g., Sitko et al. 2011), while $x_{\max }$ has been selected to range from $10 \mathrm{~cm}$ to $10 \mathrm{~m}$ (e.g., Harmon et al. 2011).

We can now compare the approach based on the light curve (providing an upper limit) with that based on the tail's brightness (offering a lower limit). Turning to Fig. 6, we find that at the time of cataclysmic fragmentation on December 17.6 UT, at a heliocentric distance of $r_{\text {rel }}=0.144 \mathrm{AU}$, the corrected magnitude $H_{\Delta}^{\prime}\left(r_{\text {rel }}\right)=-1.6$, which implies that $\left(H_{\text {eff }}\right)_{\max }=+2.6$ and an upper limit $\left(X_{\text {dust }}\right)_{\max }=3.5 \times 10^{6} \mathrm{~km}^{2}$. The estimated uncertainty is at least \pm 30 percent. For nine different combinations of the parameters $k$ and $x_{\max }$, the resulting upper limits on the mass $\left(\mathcal{M}_{\text {dust }}\right)_{\max }$ and the effective diameter $\mathcal{D}_{\max }$ are listed in Table 5 .

The source of the best information that we have been able to find for the brightness of the dust tail of $\mathrm{C} / 2011$ W3 at this early phase of investigation is an attempt undertaken by Seargent (2011) on 2011 December 24.69 UT. Using an out-of-focus method of brightness estimation for comparison stars, he determined the total brightness of the dust tail in the field of view (of an estimated diameter of $\sim 3^{\circ}$ ) of his $25 \times 100$ binocular telescope at three angular distances $\ell$ from the head that he estimated at magnitude 4.8. The results of Seargent's observation are presented in columns 1 to 3 of Table 6 . The four subsequent columns provide information on the particles and the expected tail orientation at each distance from the head for two assumed times of release. The geocentric and heliocentric distances, the phase angle, and the resulting effective visual magnitude $H_{\mathrm{eff}}(\ell)$, all of which are - to the given precision - independent of the choice for the time of release, are listed in 
Table 6

Total Brightness of Dust Tall of Comet C/2011 W3 on 2011 December 24.69 Ut (Estimates by D. Seargent)

\begin{tabular}{|c|c|c|c|c|c|c|c|c|c|c|}
\hline \multirow{2}{*}{$\begin{array}{l}\text { Angular } \\
\text { distance } \ell \\
\text { from head }\end{array}$} & \multirow{2}{*}{$\begin{array}{c}\text { Visual } \\
\text { magnitude } \\
\text { estimate }\end{array}$} & \multirow{2}{*}{$\begin{array}{c}\text { Observed } \\
\text { tail } \\
\text { width }\end{array}$} & \multicolumn{2}{|c|}{ Parameter $\beta$ for $\left(t_{\mathrm{rel}}\right)^{\mathrm{a}}$} & \multicolumn{2}{|c|}{ Position angle for $\left(t_{\mathrm{rel}}\right)^{\mathrm{a}}$} & \multicolumn{2}{|c|}{ Distance (AU) from } & \multirow{2}{*}{$\begin{array}{l}\text { Phase } \\
\text { angle }\end{array}$} & \multirow{2}{*}{$\begin{array}{c}\text { Effective } \\
\text { magnitude } \\
H_{\text {eff }}(\ell)\end{array}$} \\
\hline & & & +0.4 day & +1.6 days & +0.4 day & +1.6 days & Earth & Sun & & \\
\hline $4^{\circ}$ & 4 & $0^{\circ} .5$ & 0.08 & 0.36 & $237^{\circ} .9$ & $231^{\circ} .7$ & 0.64 & 0.51 & $117^{\circ}$ & 6.8 \\
\hline 8 & 4.5 & 1 & 0.17 & 0.71 & 238.4 & 231.9 & 0.62 & 0.56 & 113 & 7.0 \\
\hline 23 & 5 & 2 & 0.50 & 2.09 & 239.8 & 232.4 & 0.58 & 0.72 & 97 & 6.6 \\
\hline $28^{\mathrm{b}}$ & $\therefore$. & $\ldots$ & 0.62 & 2.58 & 240.1 & 232.6 & 0.58 & 0.77 & 93 & $\ldots$ \\
\hline
\end{tabular}

${ }^{a}$ Assumed time of dust release reckoned from the time of the comet's perihelion passage.

b Length of the tail seen by Seargent with the naked eye.

c According to Seargent's report, over its last $5^{\circ}$ the tail was very faint and difficult with the naked eye and could not be traced in the $25 \times 100$ binocular telescope.

columns 8 to 11 . The fundamental conclusion from the tabulated values of $H_{\text {eff }}(\ell)$ is that the total brightness in each band of the tail was virtually independent of the distance from the head and equal to $6.8 \pm 0.2$ : the surface brightness was decreasing with distance, but the tail width was increasing. And since Seargent could detect no tail with his binocular telescope beyond $\sim 23^{\circ}$ from the head, we assume in our conservative estimate that it ended there. The whole tail was then brighter by a factor of $\frac{23}{3}$, or 2.2 magnitudes, than the average in each $3^{\circ}$ band, which leads to a lower limit of $\left(H_{\text {eff }}\right)_{\min }=4.6$, or 2 magnitudes fainter than indicated by $\left(H_{\text {eff }}\right)_{\max }$ from the light curve. Consequently, the lower limit on the total cross sectional area of the comet's dust released after perihelion is $\left(X_{\text {dust }}\right)_{\min }=0.6 \times 10^{6} \mathrm{~km}^{2}$. To obtain lower limits on the mass and diameter estimates, the values in Table 5 should be multiplied by 0.16 and 0.54 , respectively.

It appears that the derived lower limit is relatively secure. Seargent's value for the brightness of the head is about 0.9 magnitude below the light curve in Fig. 6 (suggesting a personal magnitude scale difference) and our truncation of the tail's length also adds a few tenths of a magnitude, because it implies a loss of light in the binocular magnitudes relative to those obtained with the unaided eye. A fairly narrow range resulting from the two entirely independent approaches applied is encouraging and may indicate that upon the terminal breakup all atomic sodium escaped very rapidly, leaving hardly any lasting signature in the comet's light curve, given that at 0.144 AU from the Sun the Na photoionization lifetime is less than 1 hour (Huebner, Keady, \& Lyon 1992; also: Cremonese et al. 1997, Combi, DiSanti, \& Fink 1997).

Because of the unacceptably large mass estimates, greatly exceeding $10^{13}$ grams, we suggest that the exponent $k$ of the size-distribution power law in Table 5 could not possibly be near or below 3.5. However, there are fairly good odds that the nucleus could have been as large as 150-200 meters in diameter and that it could have had a residual mass as much as $\sim 10^{12}$ grams shortly after passing through perihelion.

\section{PROPERTIES OF DUST IN THE TAIL BEFORE AND AFTER PERIHELION}

Examining eleven of the brighter SOHO Kreutz-system comets with prominent tails, all on their way to perihelion between 1996 and 1998, Sekanina (2000) noticed that the narrow and nearly straight tails deviated strikingly from the antisolar direction. The highest solar radiation- pressure accelerations, to which the dust in the tails was subjected, was found to have been $\beta=0.6$, suggesting the presence of a population of submicron-sized grains dielectric in nature, most probably silicates (e.g., Sekanina et al. 2001 and the references listed there in Sec. II.A.2). The sampled comets also showed rather consistently that the peak production of dust occurred some $20-30 R_{\odot}$ from the Sun, about 1 day before perihelion, and that activity essentially terminated shortly afterwards. Detailed modeling of one of these objects showed that its tail was a syndyname of $\beta=0.6$ far from the head, but a synchrone referring to a release time of 0.8 day before perihelion $\left(19 R_{\odot}\right.$ from the Sun), near the head. A sharp bend or knee, but no gap, separated the two parts of the tail. The author suggested that the time of the abrupt termination was possibly nuclear-size dependent (Sekanina 2000).

To learn more about this possible relation and about the dust-emission pattern of comet C/2011 W3, we have examined the properties of its tail both before and after perihelion. Two images taken by the C3 coronagraph on board the SOHO spacecraft shortly before perihelion, on December 15.504 and 15.796 UT, are reproduced in Fig. 7. Cursory inspection reveals a tail whose curvature is slight but increases with time, with no obvious knee. Two syndynames are plotted for each image in the right-hand side panel: the one on the left corresponds to $\beta=2.5$, the other to $\beta=0.6$. The two radiation-pressure accelerations have been chosen because they are of particular significance for cometary dust (e.g., Sekanina et al. 2001): the first is the highest acceleration known to affect dust in comets and is typical of strongly absorbing grains (such as carbon-rich, organic material), whereas the second, as mentioned above, is characteristic of the peak acceleration on dielectric grains (such as silicates). In both cases the particles involved are in the submicronsize range. Superposition of the syndynames on the images in Fig. 7 shows for both observation times that the tail lies largely between the two syndynames and that it is slightly less curved than either of them. A conclusion from these images alone is that $\mathrm{C} / 2011 \mathrm{~W} 3$ was releasing both dielectric and absorbing dust on its way to perihelion. The main difference between the two syndynames in Fig. 7 is that at a given distance from the nucleus, the dust on the syndyname of $\beta=0.6$ was released much earlier than that on the syndyname of $\beta=2.5$.

It is instructive to compare the comet's appearance in the two preperihelion images with very different views offered shortly after perihelion by the SOHO C2 corona- 
SOHO IMAGES AND MODELS OF DUST TAIL OF C/2011 W3 ON DEC. 15
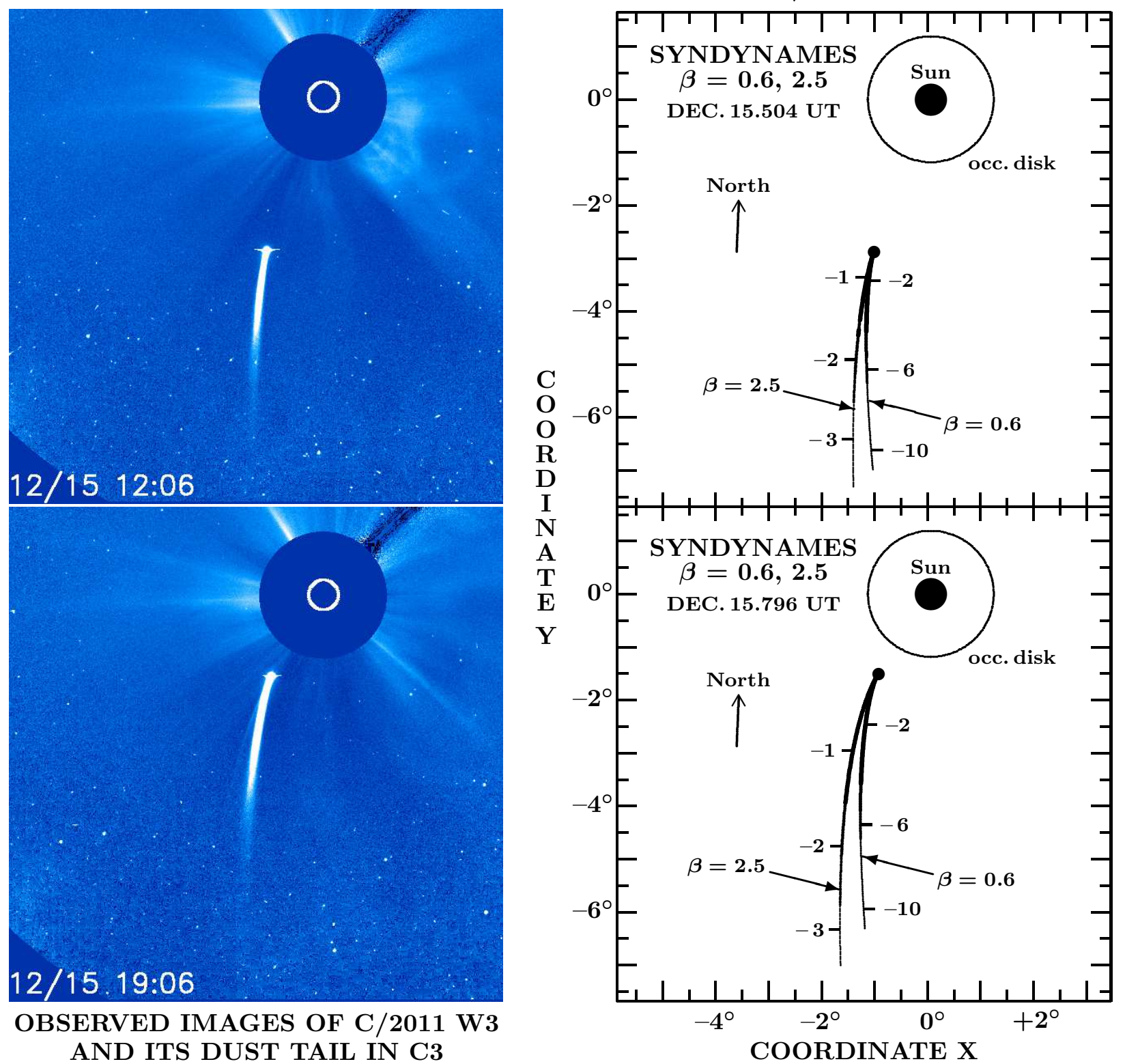

Figure 7. Comet C/2011 W3 with its dust tail shortly before reaching perihelion, as imaged by the C3 coronagraph on board the SOHO spacecraft. The upper image was taken on December $15.504 \mathrm{UT}$, or 0.508 day before perihelion, the lower image on December 15.796 UT, or 0.216 day before perihelion. In the panels next to the images the observations are compared with two syndynames; the one for $\beta=2.5$ is slightly to the left of the other, which refers to $\beta=0.6$. The syndynames are calibrated by the times of dust release, reckoned in days from the time of the comet's perihelion passage (the negative numbers indicate days before the comet's perihelion). The panels also provide the scale and orientation of the images. We note that in both images the tail displays a slightly lesser curvature than the syndynames, but lies generally between them, which implies that the comet released submicron-sized dust of both dielectric and strongly absorbing nature. (Image credit: ESA/NASA/LASCO consortium.)

graph in combination with the COR1 and COR2 coronagraphs on board the STEREO-A and STEREO-B spacecraft. The three selected images are of course merely snapshots of continuous changes in the comet's figure that are fully revealed only by the dynamic, time-lapse imaging that involves the entire data set. Nevertheless, given the spatial distribution of the three spacecraft, the stereoscopic quality of the gained information makes even snapshot views extremely valuable.

Figure 8 shows the comet's head and a part of its tail in a frame taken with the $\mathrm{C} 2$ coronagraph on December 16.117 UT, or 0.105 day after perihelion. The bright tail, to the south-southeast of the Sun, is entirely disconnected from the head. The panel to the right of the image, showing the complete syndyname $\beta=0.6$, suggests that, contrary to the observation, the tail should have reappeared to the northwest of the occulting disk. The comet's head, although clearly saturated and displaying some "blooming", is essentially stellar in appearance. This means that no detectable postperihelion tail 
SOHO C2 IMAGE AND MODEL OF DUST TAIL OF C/2011 W3 ON DEC. 16
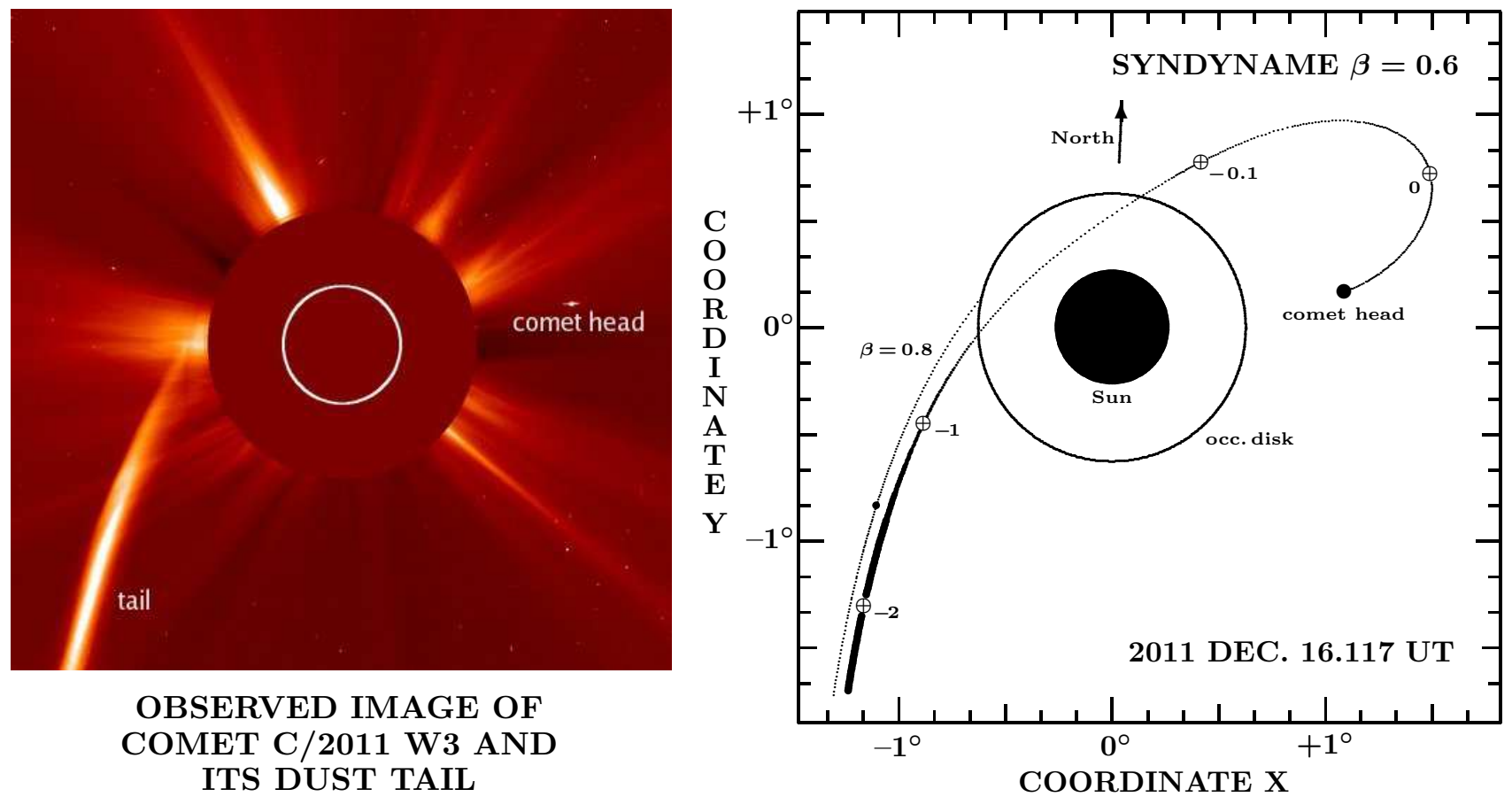

Figure 8. Appearance of comet C/2011 W3 and its dust tail in an image taken with the C2 coronagraph on board the SOHO spacecraft on December 16.117 UT, or 0.105 day after perihelion. The tail, to the south-southeast of the Sun, is seen to be completely disconnected from the comet's head, to the west of the Sun. The head's image is saturated (with some blooming being apparent) but with no tail extension. The tail lies entirely between the syndynames $\beta=0.6$ and 0.8 , as shown in the panel to the right. The first of the two syndynames is plotted thicker and is calibrated by the times of dust release, reckoned in days from the time of the comet's perihelion passage, pinpointing the locations of particles released at perihelion and $0.1,1$, and 2 days before perihelion. The dot on the segment of the syndyname $\beta=0.8$ identifies the location of dust released 1 day before perihelion. We note that the entire northwestern branch of the tail is missing. The tail's brightness is largely determined by forward scattering of sunlight by dust, as the phase angle increases toward the bottom of the image. The scale and orientation of the image are the same as those of the panel. (Image credit: ESA/NASA/LASCO consortium.)

developed by the time the image was taken, about 0.1 day after perihelion.

An example of the peculiar appearance of $\mathrm{C} / 2011 \mathrm{~W} 3$ is presented in Fig. 9, which shows the comet imaged with the COR2 coronagraph on board the STEREO-A spacecraft on December 16.246 UT, or 0.234 day after perihelion. Unlike in the SOHO's C2 coronagraph, the comet now consists of three components: the head with a short wisp of a new tail that must have begun to develop before 0.2 day after perihelion - to the eastnortheast of the Sun and two separate branches of the old tail, one to the southeast and the second to the northwest of the Sun. The southeastern branch is relatively sharp but faint, a far cry from its luster in Fig. 8. It can be matched with the syndyname $\beta=0.6$, but the resolution is poor as the syndynames along this tail are "crowded". The northwestern branch is blob shaped. Its first view in this COR2 coronagraph coincided approximately with the re-appearance of the comet's head from behind the occulting disk, nearly 3 hours after perihelion. In projection onto the plane of the sky, this branch may unfortunately have been superposed on top of a weak but broad coronal mass ejection. Because of this interference, the tail's exact contours are hard to establish, but the syndyname $\beta=0.6$ seems to be again involved with the feature. The blob terminates just before crossing the synchrone for a release time of 0.1 day before perihelion. Again, there is no tail in the areas corresponding to re- lease times near perihelion. Neither of the two branches becomes obvious in any postperihelion image taken with the COR1 coronagraph of the STEREO-A spacecraft.

In a long series of images taken with the COR1 and COR2 coronagraphs on board the STEREO-B spacecraft during much of the first day after perihelion, the look of the comet with its tail is downright bizarre. In fact, hours before the comet's head emerged from behind the occulting disk to the west-southwest, a second branch of the tail began to show up as a steadily growing sharp spike to the northeast of the Sun, joining the southwestern branch that had thrived since preperihelion times. In addition, the comet's head, after it emerged, was, just as in Fig. 9, disconnected from either of the two branches of the tail. Overall, therefore, the comet again consisted of three discrete components, as seen in Fig. 10. The displayed image was taken with the COR2 coronagraph on December 16.517 UT, or 0.505 day after perihelion. The syndyname $\beta=0.6$ provides an excellent fit to both branches of the tail simultaneously, although the syndynames are again fairly "crowded" along much of the tail. This image shows that the northeastern branch extends to a point that is populated by dust released $\sim 0.1$ day before perihelion, in agreement with the result from Fig. 9. Careful inspection of the comet's head reveals its elongation similar to that detected in the STEREO-A image.

To extract information on the dust particles that populate the tail, we have examined more closely the or- 
STEREO-A IMAGE AND MODEL OF DUST TAIL OF C/2011 W3 ON DEC. 16
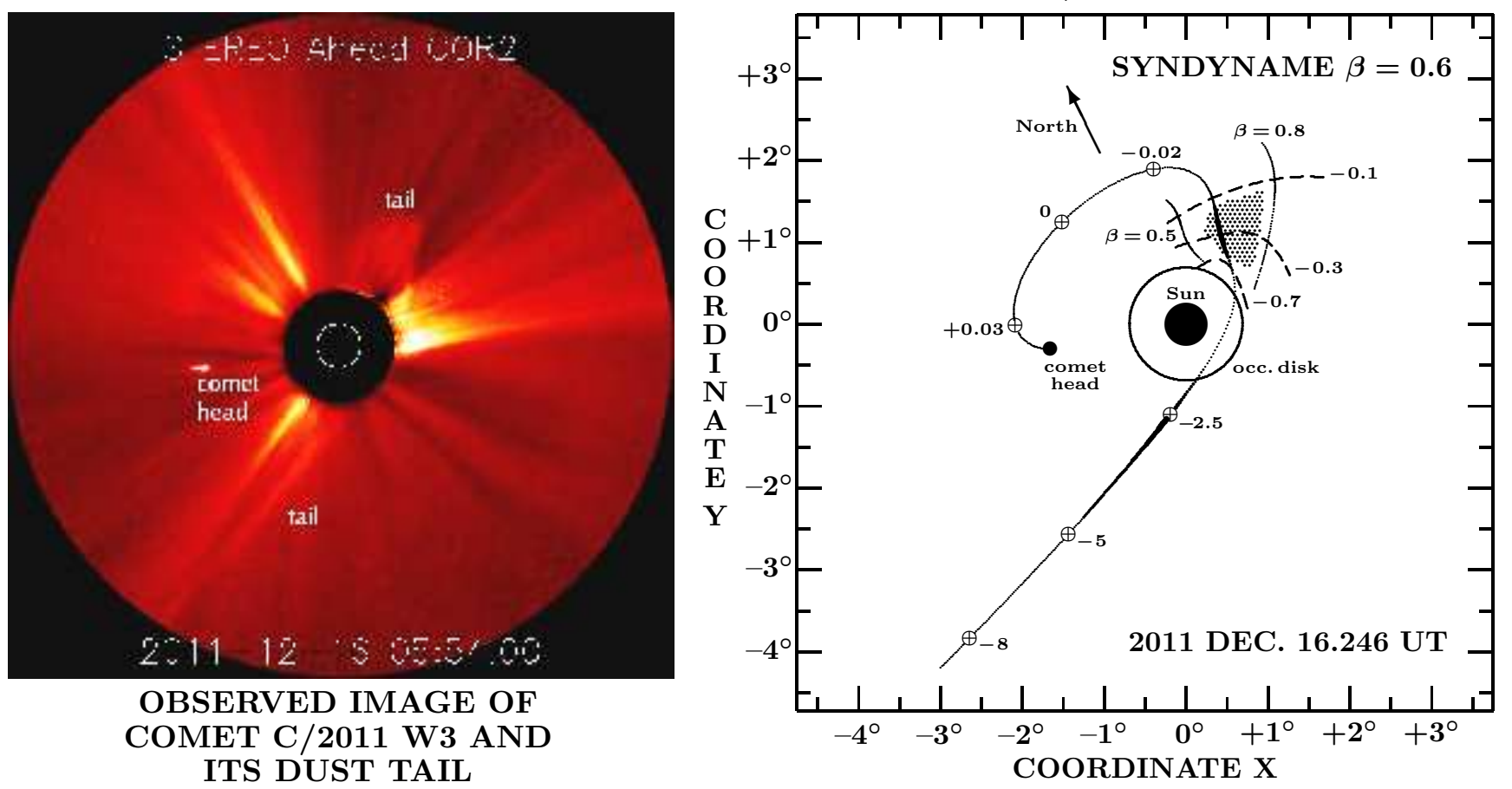

Figure 9. Peculiar appearance of comet C/2011 W3 and its dust tail in an image taken with the COR2 coronagraph on board the STEREO-A spacecraft on December 16.246 UT, or 0.234 day after perihelion. The tail has two branches located, respectively, to the southeast and northwest of the Sun, while the comet head's site is nearly to the east of the Sun. The tail's southeastern branch is faint because of backscatter of sunlight, while the blob-shaped northwestern branch appears to be at least in part contaminated by a relatively weak, but broad coronal mass ejection. The panel to the right identifies the locations on the syndyname $\beta=0.6$, which are populated by dust released from the nucleus at six different times between 8 days before perihelion and 0.03 day after perihelion. Three additional release times - $0.1,0.3$, and 0.7 day before perihelion - are identified by the synchrones drawn by the broken curves. The panel also shows the area of the tail's blob to the northwest of the Sun and short segments of the syndynames for $\beta$ equaling 0.5 and 0.8 . No tail is detected for release times between 0.1 day before perihelion and about 0.1 day after perihelion, but the head's image is elongated in the direction away from the Sun, suggesting that a postperihelion tail already began to develop during a couple of hours before the frame was taken. (Image credit: NASA/SECCHI consortium.)

bital properties of dust particles along the syndyname $\beta=0.6$ with the help of Table 7 , which lists their perihelion distance, the eccentricity, and the perihelion time as a function of $\beta$ and the time of release from the comet. The numbers along this syndyname in Figs. 8-10 are the times of release in days from the comet's perihelion time. While it is rather clear that the bright branch of the tail to the south-southeast in Fig. 8 is identical with the fairly pale southeastern branch in Fig. 9 and with the gleaming southwestern branch in Fig. 10, the reason for the enormous differences in brightness is not obvious. The orbital elements of the dust particles in Table 7 allow one to calculate the geometry for the times of the three images and to find out that the culprit is forward scattering of sunlight. For example, the particles in the bright portion of the tail in Fig. 8, located on the syndyname $\beta=0.6$ and released 2 days before perihelion, were at the time of observation nearly $7 R_{\odot}$ from the Sun and their phase angle was $113^{\circ}$, while for the particles that left the nucleus 1 day before perihelion, although they were only $3.6 R_{\odot}$ from the Sun, the phase angle was $90^{\circ}$ and for those released 0.7 day before perihelion and located near the edge of the occulting disk, the phase angle was only $70^{\circ}$, the reason why this part of the tail looks faint. For the same reason, the brightness of the tail's southeastern branch in Fig. 9 cannot compare with that of the southwestern branch in Fig. 10 even though it is the same feature: at the location of particles on the syndyname $\beta=0.6$ released 5 days before perihelion, in the middle of this tail, the phase angle is $55^{\circ}$ in Fig. 9, but $109^{\circ}$ in Fig. 10.

It is further noted from Table 7 that particles on that same syndyname that were released earlier than 2.5 days before perihelion did not reach their perihelion points until after December 16.5 UT and, at the times the images in Figs. 8-10 were taken, were still on their way toward the Sun. By contrast, particles released later than 0.5 day before perihelion reached their perihelion points no later than about December 16.1 UT and were therefore in all three images already moving away from the Sun. This is important because the relevant perihelion distances are very small. Table 7 shows that on the syndyname $\beta=0.6$ this minimum distance drops from $2.37 R_{\odot}$ for dust that left the nucleus 0.5 day before perihelion to $2.21 R_{\odot}$ for dust that left 0.3 day before perihelion and to $1.81 R_{\odot}$ for dust that left 0.1 day before perihelion. And, as expected, it is equal to the perihelion distance of the comet, $1.19 R_{\odot}$, for dust that left at perihelion. Since the phase angle for particles on the syndyname $\beta=0.6$ released at perihelion is in Fig. 8 equal to $88^{\circ}$, nearly identical with the phase angle for particles released 0.7 day before perihelion (see above), the utter absence of 
STEREO-B IMAGE AND MODEL OF DUST TAIL OF C/2011 W3 ON DEC. 16
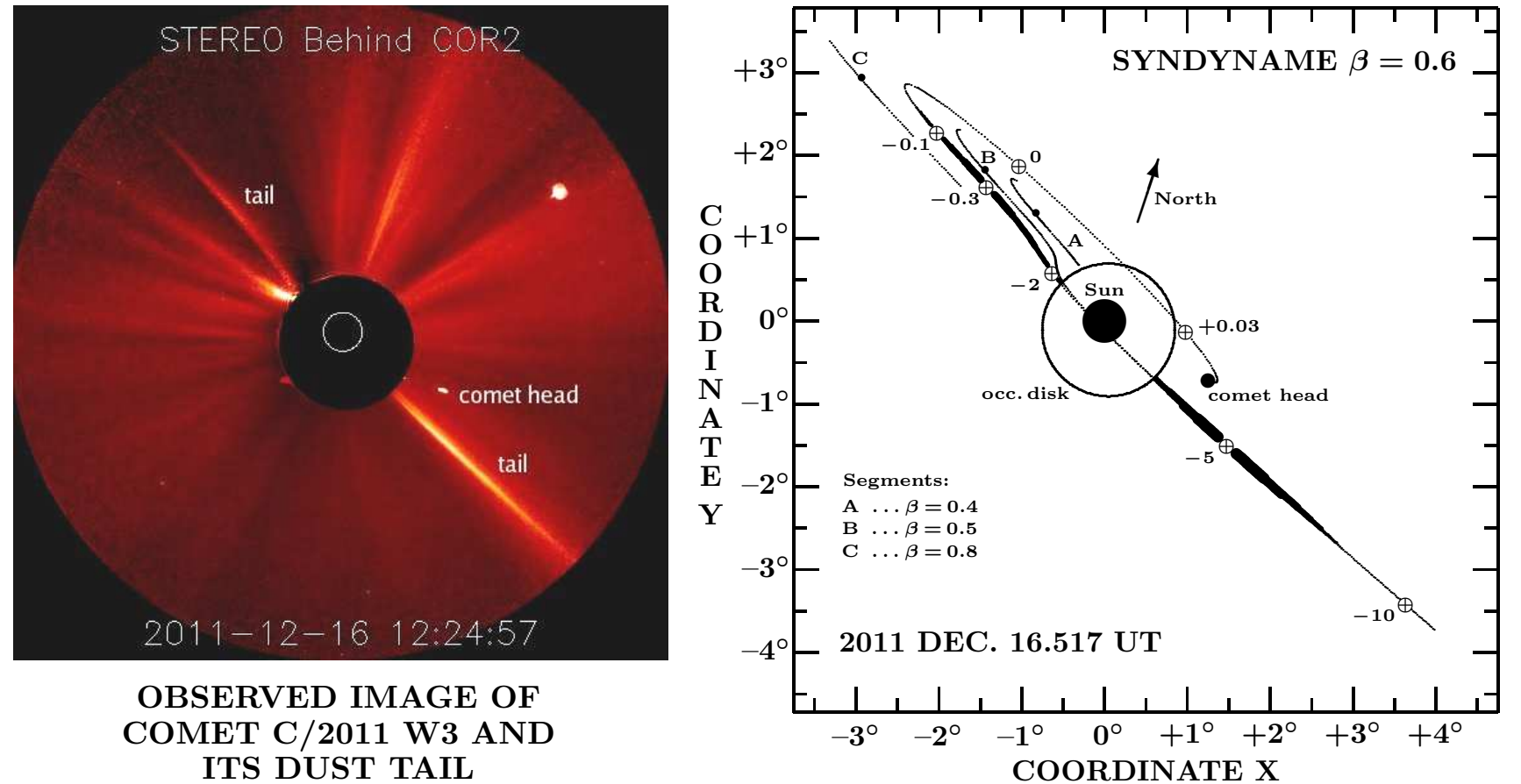

Figure 10. Bizarre appearance of comet C/2011 W3 and its dust tail in an image taken with the COR2 coronagraph on board the STEREO-B spacecraft on December 16.517 UT, or 0.505 day after perihelion. The tail has two branches located, respectively, to the southwest and northeast of the Sun, while the comet's head is to the west-southwest of the Sun and north of the brightest part of the tail's southwestern branch. Careful inspection shows that the head's image is elongated along the line with the Sun, evidence that a new, postperihelion tail is being developed. The entire tail is fitted with a syndyname of $\beta=0.6$, as shown in the panel that also provides the scale and orientation of the image. Only the heavily-drawn segments of the syndyname are seen as the tail in the image. The syndyname is calibrated by the times of dust release, reckoned in days from the time of the comet's perihelion passage (a negative number means before perihelion, and vice versa). Brightness-wise, the southwestern branch dominates because of forward scattering of sunlight. A broad secondary maximum is noted on the northeastern branch at a location corresponding to a release time of about 0.3 day before perihelion (December $15.7 \mathrm{UT}$ ). The tail then rapidly fades and disappears for a release time near 0.1 day before perihelion, suggesting an attentuation of activity hours before perihelion. Short segments of the syndynames $\beta=0.4,0.5$, and 0.8 are also plotted, marked as A, $\mathrm{B}$, and $\mathrm{C}$, respectively, to illustrate a general deficit of dust with accelerations different from 0.6 . The dots on these syndyname segments show the locations of particles released 0.1 day before perihelion. The northeastern tail's boundary is sharper to the south than to the north, indicating that dust with $\beta \gg 0.6$ is more scarce than that with $\beta<0.6$. No tail is apparent along the wide swath of space referring to release times during the first hours after perihelion. (Image credit: NASA/SECCHI consortium.)

dust from emissions at perihelion has nothing to do with particle sunlight scattering effects. And since Figs. 8-10 consistently show the absence of a tail for release times between approximately 0.1 day before perihelion and 0.1 day after perihelion, that is, for particles whose perihelion distances were always smaller than $\sim 1.8 R_{\odot}$, the most likely explanation for this "activity attenuation" is the effect of sublimation of microscopic dust near the Sun, as discussed in detail in the following section.

Probably the most diagnostic evidence for this effect is offered by the northeastern branch of the tail in Fig. 10 , which is made up of the dust that left the nucleus later than 2.2 days before perihelion. At the time of observation, these particles were more than $3 R_{\odot}$ from the Sun; the phase angle reached $110^{\circ}$ for those at the edge of the occulting disk but dropped rapidly along the tail to $70^{\circ}$ about $1^{\circ}$ from the Sun and was nearly constant around $50^{\circ}$ along much of the rest of it. The apparent brightening at the location of particles released $\sim 0.3$ day before perihelion is thus likely to reflect their increased production around that time. Table 7 indicates that the dust at the edge of the occulting disk reached perihelion, at $2.7 R_{\odot}$ from the Sun, only about 0.45 day after the nucleus, while the dust at the far end of this tail's branch was nearest the Sun, at merely $\sim 1.8 R_{\odot}$, practically simultaneously with the nucleus. This means that at the time of observation all particles along the northeastern tail were already moving away from the Sun. The fading and disappearance of this tail at a location populated by submicron-sized particles released $\sim 0.08-0.1$ day before perihelion and moving in highly hyperbolic orbits (eccentricity $\sim 2.3$ or higher) strongly indicate that their perihelion distance of $\sim 1.8 R_{\odot}$ is indeed the limit at which dust begins to sublimate profusely.

The syndyname of $\beta=0.6$ continues to extend in Fig. 10 a little farther from the Sun than the disappearing northeastern tail and then turns sharply back, almost $180^{\circ}$, running nearly parallel to the tail, in part behind the occulting disk, all the way to the comet's head. No trace of dust debris, all of which moved in orbits with perihelion distances smaller than $\sim 1.8 R_{\odot}$, is - just as in Figs. 8 and 9 - detected along this arc of the syndyname. Thus, no particles released between about 0.1 day before perihelion and at least 0.1 day after perihelion appear to have survived, again pointing to $\sim 1.8 R_{\odot}$ as the sublimation cutoff. 
Table 7

Orbital Elements of Dust Particles in the Tail of Comet C/2011 W3

\begin{tabular}{|c|c|c|c|c|c|c|c|c|c|c|c|c|c|c|c|}
\hline \multirow{3}{*}{$\begin{array}{l}\text { Time } \\
\text { of } \\
\text { release } \\
\left(\text { days }^{\mathrm{b}}\right) \\
\end{array}$} & \multirow{3}{*}{$\begin{array}{c}\text { Distance } \\
\text { from } \\
\text { Sun } \\
\left(R_{\odot}\right) \\
\end{array}$} & \multicolumn{14}{|c|}{ Orbital elements of released dust particles ${ }^{\mathrm{a}}$ as function of parameter $\beta$} \\
\hline & & \multicolumn{5}{|c|}{ Perihelion distance $\left(R_{\odot}\right)$} & \multicolumn{4}{|c|}{ Orbit eccentricity $^{\mathrm{c}}$} & \multicolumn{5}{|c|}{ Perihelion time $\left(\right.$ days $\left.^{\mathrm{b}}\right)$} \\
\hline & & 0.3 & 0.6 & 1.0 & 1.5 & 2.5 & 0.3 & 0.6 & 1.5 & 2.5 & 0.3 & 0.6 & 1.0 & 1.5 & 2.5 \\
\hline-10 & & 1.69 & 2.87 & 11.40 & 38.50 & & & & 1.1240 & & +0.748 & +1 & & +4.1 & -0.038 \\
\hline-5 & 67.94 & 1.69 & 2.81 & 9.01 & 24.86 & 41.58 & 1.0212 & 1.1237 & 1.1921 & 1.038 & +0.387 & +0.967 & +2.248 & +1.931 & -0.0 \\
\hline-2 & 36.37 & 1.67 & 2.69 & 6.59 & 14.18 & 22.60 & 1.0393 & 1.2212 & 1.3368 & 1.0704 & +0.164 & +0.407 & +0.816 & +0.653 & -0.106 \\
\hline-1 & 22.51 & 1.65 & 2.55 & 5.19 & 9.41 & 14.26 & 1.0628 & 1.3396 & 1.5074 & 1.1116 & +0.087 & +0.206 & +0.356 & +0.259 & -0.095 \\
\hline-0.5 & 13.80 & 1.62 & 2.37 & 4.06 & 6.34 & 9.01 & 1.1007 & 1.5154 & 1.7536 & 1.1766 & +0.044 & +0.098 & +0.139 & +0.082 & -0.078 \\
\hline-0.2 & 7.09 & 1.56 & 2.07 & 2.91 & 3.83 & 4.94 & 1.1884 & 1.8778 & 2.2456 & 1.3224 & +0.015 & +0.027 & +0.024 & -0.002 & -0.056 \\
\hline-0.1 & 4.22 & 1.48 & 1.81 & 2.24 & 2.67 & 3.17 & 1.3009 & 2.2894 & 2.7905 & 1.5023 & +0.004 & +0.003 & -0.004 & -0.018 & -0.040 \\
\hline 0 & 1.19 & 1.19 & 1.19 & 1.19 & 1.19 & 1.19 & 1.8570 & 3.9998 & 4.9999 & 2.3333 & 0.000 & 0.000 & 0.000 & 0.000 & 0.000 \\
\hline$+0.1^{\mathrm{d}}$ & 4.22 & 1.48 & 1.81 & 2.24 & 2.67 & 3.17 & 1.3009 & 2.2894 & 2.7905 & 1.5023 & -0.004 & -0.003 & +0.004 & +0.018 & +0.040 \\
\hline+0.3 & 9.55 & 1.59 & 2.21 & 3.38 & 4.77 & 6.44 & 1.1428 & 1.6955 & 2.0001 & 1.2473 & -0.025 & -0.051 & -0.060 & -0.022 & +0.066 \\
\hline
\end{tabular}

${ }^{a}$ Release (ejection) velocity assumed to be zero.

$\mathrm{b}$ Reckoned from the time of perihelion passage of the comet; minus sign means before perihelion, and vice versa.

c For $\beta=1$ (motion in a straight line), the eccentricity is by definition infinitely large regardless of the release time.

$\mathrm{d}$ This entry is included to illustrate, by comparison with the entry -0.1 day, the symmetry with respect to perihelion.

The panel in Fig. 10 also displays segments of the syndynames $\beta=0.4,0.5$, and 0.8 for a part of the northeastern tail, where they do no overlap with the syndyname $\beta=0.6$. A clearly apparent property of this tail branch is its more gradual fading to the north of its sharp edge than to the south, where the drop is abrupt. Dust with $\beta<0.6$, located to the north of the edge, has the perihelion distances systematically smaller, in agreement with the more limited presence of surviving dust than at $\beta=0.6$. The tail no longer appears to contain particles with the acceleration parameter $\beta$ significantly exceeding 0.6 , which should be located to the south.

On the other hand, the tail's bright southwestern branch in Fig. 10 was made up of dust that left the nucleus between 8 and 3.6 days before perihelion. At the time of observation these particles were between $16 R_{\odot}$ and $5 R_{\odot}$ from the Sun. Table 7 shows that they passed through perihelion between 1.5 and 0.7 days after the comet, so they were all still approaching the Sun in Fig. 10. And, as already alluded to above, this southwestern branch of the tail looks bright because of effects of forward scattering of sunlight, the phase angle varying from $93^{\circ}$ for dust released 8 days before perihelion to $134^{\circ}$ for dust released 3.6 days before perihelion. At perihelion, the distance from the Sun was from $2.77 R_{\odot}$ to $2.86 R_{\odot}$.

The comet head's elongation in Fig. 10, already mentioned above, indicates that dust particles were released from the nucleus starting about 4 hours, or slightly less than 0.2 day, after perihelion, and were therefore $\sim 8$ hours old at the time of observation. This new, postperihelion tail should have indeed extended from the nucleus in the antisolar direction. If consisting of particles with $\beta=0.6$, its length at the time the image was taken should have been between $4^{\prime}$ and $6^{\prime}$.

There is no evidence in Figs. 8-10 for dust with $\beta \gtrsim 1$. The question of what happened to it is again answered with the help of Table 7, which conveys two important facts on the whereabouts of particles in convex hyperbolic orbits: (i) those released from the comet up to about 2 days before perihelion moved in orbits with perihelion distances always exceeding (often considerably)
$9 R_{\odot}$, whereas (ii) more recent ones passed through perihelion nearly simultaneously with the comet, so that at the times the images in Figs. 8-10 were taken this dust already was at heliocentric distances much larger than the perihelion distance. In summary, dust particles with $\beta \gtrsim 1$ did not contribute to the tail's postperihelion brightness because of their dispersal over a large volume of space and reduced light scattering efficiency far from the Sun.

To address the issue of what kind of dust was released by comet C/2011 W3 after perihelion, we collected information on the tail length in the period of time from late December 2011 to mid-March 2012. A selection of 54 reported photographic and visual observations is listed in Table 8. Nearly all tail lengths from times after December 21 can be explained by submicron-sized debris from the event of December 17.6 UT (Sec. 5), if the radiationpressure accelerations $\beta$ of up to 2.5 are allowed. On the other hand, constraining $\beta$ to 0.6 requires that in most cases the tail (or at least its far reaches) derive from the activity prior to the event of December 17. To distinguish between the two scenarios, it will be necessary to measure accurately the position angles of the tail, which after December 26 became almost perfectly straight. At present we are unaware of any such measurements.

At the other end of the particle-size spectrum, a question arises as to how large the dust debris from the nucleus disintegration event of December 17 must be in order that it be released into elliptical orbits and not be lost to interstellar space. This can readily be determined by equating the orbital velocities of the comet and the debris on December 17.6 UT. For the debris to move below the escape limit, one requires that its $\beta_{\text {ell }}$ be less than $\beta_{\text {par }}$ for the parabolic limit, which follows from a condition

$$
\frac{2}{r_{\text {rel }}}-\left(\frac{1}{a}\right)_{\text {comet }}=\left(1-\beta_{\text {par }}\right) \frac{2}{r_{\text {rel }}}
$$

where $(1 / a)_{\text {comet }}$ is the inverse semi-major axis of the comet's orbit and $r_{\text {rel }}$ is the heliocentric distance at the time of release, $r_{\text {rel }}=0.144 \mathrm{AU}$ (Sec. 5). Thus, for the 
Table 8

Selection of Reported Postperihelion Tail Lengths of C/2011 W3 and Lengths Derived for Two Parameters $\beta_{0}$

\begin{tabular}{|c|c|c|c|c|c|c|c|c|}
\hline \multirow{2}{*}{\multicolumn{2}{|c|}{$\begin{array}{c}\text { Observation } \\
\text { time } \\
\text { (UT) }\end{array}$}} & \multirow{2}{*}{$\begin{array}{l}\text { Reported } \\
\text { length } \\
\text { of tail }\end{array}$} & \multicolumn{2}{|c|}{ Predicted length ${ }^{\mathrm{a}}$} & \multicolumn{2}{|c|}{ Required release time ${ }^{\mathrm{b}}(\mathrm{UT})$} & \multirow[b]{2}{*}{ Observer $^{\mathrm{c}}$} & \multirow[b]{2}{*}{ Reference $^{\mathrm{d}}$} \\
\hline & & & $\beta_{0}=2.5$ & $\beta_{0}=0.6$ & $\beta_{0}=2.5$ & $\beta_{0}=0.6$ & & \\
\hline \multirow{28}{*}{2011 Dec. } & 21.07 & $10^{\circ}$ & 7.2 & 1.8 & Dec. 17.3 & Dec. 16.4 & N. Wakefield & Mailing list \\
\hline & 21.30 & $>5$ & 8.1 & 2.0 & $\ldots \ldots \ldots$ & $<$ Dec. 16.8 & ${ }^{*}$ W. Souza & Green $(2012 a)$ \\
\hline & 21.7 & 13 & 9.8 & 2.4 & Dec. 17.3 & Dec. 16.4 & L. Barnes & Mailing list \\
\hline & 21.73 & 14.3 & 10.0 & 2.4 & Dec. 17.25 & Dec. 16.36 & R. McNaught & $"$ \\
\hline & 22.30 & 10 & 12.7 & 3.1 & $\ldots \ldots \ldots$ & Dec. 16.6 & ${ }^{*}$ W. Souza & Green $(2012 a)$ \\
\hline & 22.30 & 13 & 12.7 & 3.1 & Dec. 17.57 & Dec. 16.5 & *A. Amorim & $"$ \\
\hline & 22.32 & 20 & 12.8 & 3.1 & Dec. 17.15 & Dec. 16.3 & ${ }^{*}$ M. Goiato & $"$ \\
\hline & 22.7 & 16 & 14.8 & 3.6 & Dec. 17.5 & Dec. 16.4 & L. Barnes & Mailing list \\
\hline & 22.7 & 15 & 14.8 & 3.6 & Dec. 17.58 & Dec. 16.5 & ${ }^{*}$ D. Seargent & $"$ \\
\hline & 23.31 & 15 & 18.3 & 4.5 & $\ldots \ldots \ldots$ & Dec. 16.55 & ${ }^{*}$ M. Goiato & Green (2012a) \\
\hline & 23.7 & 22 & 20.7 & 5.1 & Dec. 17.5 & Dec. 16.4 & J. Tilbrook & Mailing list \\
\hline & 23.72 & 21.7 & 20.8 & 5.1 & Dec. 17.55 & Dec. 16.4 & R. McNaught & $"$ \\
\hline & 24.32 & 20 & 24.8 & 6.1 & 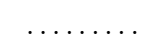 & Dec. 16.5 & *M. Goiato & Green $(2012 a)$ \\
\hline & 24.69 & 28 & 27.4 & 6.8 & Dec. 17.57 & Dec. 16.4 & ${ }^{*}$ D. Seargent & Mailing list \\
\hline & 24.7 & 27 & 27.5 & 6.8 & $\ldots \ldots \ldots$ & Dec. 16.4 & T. Barry & $"$ \\
\hline & 25.7 & 30 & 34.7 & 8.8 & & Dec. 16.5 & J. Dunphy & $"$ \\
\hline & 26.60 & 33 & 41.2 & 10.8 & $\ldots$ & Dec. 16.5 & ${ }^{*} \mathrm{R}$. Kaufman & $"$ \\
\hline & 26.63 & 38 & 41.4 & 10.9 & $\ldots$ & Dec. 16.4 & J. Drummond & $"$ \\
\hline & 26.68 & 37 & 41.8 & 11.0 & $\ldots \ldots \ldots$ & Dec. 16.45 & ${ }^{*}$ D. Seargent & $"$ \\
\hline & 26.71 & 38 & 42.0 & 11.1 & $\ldots \ldots \ldots$ & Dec. 16.4 & M. Mattiazzo & $"$ \\
\hline & 28.28 & 30 & 52.3 & 15.1 & $\ldots \ldots \ldots$ & Dec. 16.8 & ${ }^{*}$ W. Souza & $"$ \\
\hline & 28.74 & 30 & 55.0 & 16.4 & $\ldots \ldots \ldots$ & Dec. 16.85 & ${ }^{*}$ A. Pearce & $"$ \\
\hline & 29.59 & 32 & 59.2 & 18.8 & $\ldots \ldots \ldots$ & Dec. 16.9 & R. Kaufman & $"$ \\
\hline & 29.73 & 32.0 & 59.9 & 19.2 & $\ldots \ldots \ldots$ & Dec. 16.9 & ${ }^{*}$ A. Pearce & $"$ \\
\hline & 30.71 & 25 & 63.7 & 22.1 & $\ldots \ldots \ldots$ & Dec. 17.4 & M. Mattiazzo & $"$ \\
\hline & 31.58 & 30 & 66.3 & 24.7 & $\ldots \ldots \ldots$ & Dec. 17.3 & R. Kaufman & $"$ \\
\hline & 31.67 & 45 & 66.6 & 24.9 & $\ldots \ldots \ldots$ & Dec. 16.75 & *D. Seargent & $"$ \\
\hline & 31.72 & 25 & 66.7 & 25.1 & $\ldots \ldots \ldots$ & $\ldots \ldots \ldots$ & ${ }^{*} \mathrm{R} . \mathrm{McNaught}$ & $"$ \\
\hline \multirow{24}{*}{2012 Jan. } & 1.73 & 22 & 68.8 & 28.0 & $\ldots \ldots \ldots$ & $\ldots \ldots \ldots$ & ${ }^{*} \mathrm{R} . \mathrm{McNaught}$ & $"$ \\
\hline & 2.27 & 10 & 69.6 & 29.4 & $\ldots \ldots \ldots$ & $\ldots \ldots$ & *M. Goiato & Green $(2012 a)$ \\
\hline & 2.6 & $>40$ & 69.9 & 30.3 & $\ldots \ldots \ldots$ & $<$ Dec. 17.1 & L. Barnes & Mailing list \\
\hline & 2.73 & 36 & 70.1 & 30.6 & $\ldots \ldots \ldots$ & Dec. 17.3 & ${ }^{*} \mathrm{R} . \mathrm{McNaught}$ & $"$ \\
\hline & 3.73 & 30 & 70.7 & 33.0 & $\ldots \ldots \ldots$ & $\ldots \ldots \ldots$ & ${ }^{*} \mathrm{R} . \mathrm{McNaught}$ & $"$ \\
\hline & 4.63 & 33 & 70.9 & 34.8 & $\ldots \ldots \ldots$ & $\ldots \ldots \ldots$ & R. Kaufman & $"$ \\
\hline & 4.69 & 30 & 70.9 & 35.0 & $\ldots \ldots \ldots$ & $\ldots \ldots \ldots$ & M. Mattiazzo & $"$ \\
\hline & 4.70 & 38 & 70.9 & 35.0 & $\ldots \ldots \ldots$ & Dec. 17.4 & J. Tilbrook & $"$ \\
\hline & 5.70 & 35.5 & 70.6 & 36.7 & $\ldots \ldots \ldots$ & $\ldots \ldots \ldots$ & J. Tilbrook & $"$ \\
\hline & 6.74 & 21 & 69.9 & 38.0 & $\ldots \ldots \ldots$ & $\ldots \ldots \ldots$ & R. McNaught & $"$ \\
\hline & 13.48 & $>18$ & 61.1 & 39.2 & $\ldots \ldots \ldots$ & $\ldots \ldots \ldots$ & R. Kaufman & $"$ \\
\hline & 14.48 & 46 & 59.7 & 38.7 & $\ldots \ldots \ldots$ & Dec. 17.0 & R. Kaufman & $"$ \\
\hline & 15.48 & $>45$ & 58.2 & 38.1 & $\ldots \ldots \ldots$ & $<$ Dec. 17.0 & R. Kaufman & $"$ \\
\hline & 15.5 & 47 & 58.2 & 38.1 & $\ldots \ldots \ldots$ & Dec. 16.9 & L. Barnes & $"$ \\
\hline & 16.48 & 45 & 56.8 & 37.4 & $\ldots \ldots \ldots$ & Dec. 17.0 & L. Barnes & $"$ \\
\hline & 18.50 & 37 & 54.1 & 36.0 & $\ldots \ldots \ldots$ & Dec. 17.5 & L. Barnes & $"$ \\
\hline & 20.48 & 39 & 51.6 & 34.7 & $\ldots \ldots \ldots$ & Dec. 17.2 & R. Kaufman & $"$ \\
\hline & 22.5 & 29 & 49.4 & 33.4 & $\ldots \ldots \ldots$ & $\ldots \ldots$ & L. Barnes & $"$ \\
\hline & 23.5 & 39 & 48.4 & 32.7 & $\ldots \ldots \ldots$ & Dec. 17.0 & L. Barnes & $"$ \\
\hline & 24.5 & 30 & 47.5 & 32.1 & $\ldots \ldots \ldots$ & $\ldots \ldots \ldots$ & L. Barnes & $"$ \\
\hline & 25.55 & 26 & 46.6 & 31.5 & $\ldots \ldots \ldots$ & $\ldots \ldots \ldots$ & R. Kaufman & $"$ \\
\hline & 26.52 & 31 & 45.7 & 31.0 & $\ldots \ldots \ldots$ & $\ldots \ldots \ldots$ & L. Barnes & $"$ \\
\hline & 28.52 & 17.5 & 44.2 & 30.0 & $\ldots \ldots \ldots$ & $\ldots \ldots \ldots$ & L. Barnes & $"$ \\
\hline & 30.59 & 26 & 42.8 & 29.0 & $\ldots \ldots \ldots$ & $\ldots \ldots \ldots$ & R. Kaufman & $"$ \\
\hline Feb. & 12.46 & 7 & 36.7 & 24.8 & $\ldots \ldots \ldots$ & $\ldots \ldots \ldots$ & R. Kaufman & $"$ \\
\hline Mar. & 16 & 1.2 & 28.3 & 18.9 & $\ldots \ldots \ldots$ & $\ldots \ldots \ldots$ & L. Barnes & Barnes (2012) \\
\hline
\end{tabular}

a Assuming the tail consists only of dust released during the outburst that peaked on Dec. 17.6 UT, about 1.6 days after perihelion.

b For reference, the passage through perihelion occurred on Dec. 16.01 UT.

c Asterisk preceding the observer's name indicates a visual detection.

$\mathrm{d}$ Mailing list $=$ http://tech.groups.yahoo.com/group/comets-ml messages 18968-19383. 
debris in elliptical orbits

$$
\beta_{\text {ell }}<\frac{1}{2} r_{\text {rel }}\left(\frac{1}{a}\right)_{\text {comet }}=0.000915
$$

and the particle diameters $x_{\text {ell }}$ at an assumed bulk density of $0.4 \mathrm{~g} \mathrm{~cm}^{-3}$ are $>0.3 \mathrm{~cm}$, if released during the December 17 event. The correction to the barycenter of the solar system would change this result imperceptibly. The same condition for particles released at perihelion would yield $\beta_{\text {ell }}<0.000035$ and $x_{\text {ell }}>8.2 \mathrm{~cm}$. We conclude that only coarse-grain and larger debris continues to orbit the Sun in elliptical orbits. This is a way to generate SOHO-like minicomets that move in orbits very similar to that of the comet from which they originate, except for the orbital period.

\section{SUBLIMATION OF MICROSCOPIC DUST NEAR THE SUN}

The existence of the so-called dust-free zone around the Sun, a result of profuse sublimation of interplanetary dust, has been extensively studied. The problem is well reviewed by, for example, Mann et al. (2004), based in part on earlier works by Krivov, Kimura, \& Mann (1998), Kimura, Ishimoto, \& Mukai (1997), and others. More recently this topic was also discussed by Kama, Min, \& Dominik (2009) in connection with the problem of an inner boundary of protoplanetary discs. Mann et al. (2004) list the radius of the sublimation zone for different materials, as computed by various authors. The numbers of particular interest are: $1.5-4 R_{\odot}$ for quartz (compact), $4 R_{\odot}$ for glassy carbon (both compact and fluffy), 5-6.5 $R_{\odot}$ for Mg-rich pyroxene (both modes), $10 R_{\odot}$ and $13.5-15.5 R_{\odot}$ for Mg-rich olivine (compact and fluffy, respectively), $14 R_{\odot}$ for astronomical silicate (compact), and 11-24 $R_{\odot}$ for a compact iron sphere. In most cases, however, the particles were assumed to orbit the Sun in a nearly-circular path until complete attrition, while for cometary particles moving along strongly hyperbolic orbits the critical perihelion distance is expected to be even slightly less.

The mineralogical and morphological properties of silicate grains in sungrazing comets were studied extensively by Kimura et al. (2002), who used a large set of light curves of the SOHO Kreutz minicomets as the data base. The authors concluded that the dust tails contained aggregates of submicron-sized crystalline grains, but not amorphous grains. The sublimation of fluffy olivine aggregates was proposed by them to explain the downturn on the light curves near $11-12 R_{\odot}$. An increasing outflow of pyroxene fluffy aggregates was suggested as the source for a secondary maximum on the light curves near $7 R_{\odot}$. Their hypothesis does not appear to assign any significance to the range of heliocentric distances between 1.5 and $2 R_{\odot}$.

To examine the sublimation properties of microscopic dust surviving in hyperbolic orbits with these small perihelion distances, consider a spherical dust particle of a bulk density $\rho$ and radius $a_{\text {rel }}$ released from the nucleus at time $t_{\text {rel }}$. Let the particle, which moves in a field of reduced effective gravity, sublimate at a temporally variable rate $\dot{a}_{\text {subl }}(t)$, such that at the time of observation, $t_{\text {obs }}$, the radius is reduced to $a_{\text {fin }} \ll a_{\text {rel }}$, so that

$$
a_{\mathrm{fin}}=a_{\mathrm{rel}}-\int_{t_{\mathrm{rel}}}^{t_{\mathrm{obs}}} \dot{a}_{\mathrm{subl}}(t) d t,
$$

where the sublimation rate is defined as a positive quantity. The rate $\dot{a}_{\text {subl }}$ depends strongly on the particle's equilibrium temperature $T(t)$ and can be expressed in terms of the mass sublimation rate per unit area, $\dot{\mathcal{Z}}_{\text {subl }}$ (in $\mathrm{g} \mathrm{cm}^{-2} \mathrm{~s}^{-1}$ ):

$$
\dot{a}_{\text {subl }}(T)=\frac{\dot{\mathcal{Z}}_{\text {subl }}(T)}{\rho},
$$

where $\dot{\mathcal{Z}}_{\text {subl }}$ is a function of the sublimation pressure, $\wp_{\text {subl }}(T)$,

$$
\dot{\mathcal{Z}}_{\text {subl }}(T)=\left(\frac{\mu}{2 \pi \Re T}\right)^{\frac{1}{2}} \wp_{\text {subl }}(T),
$$

$\mu$ is the molar weight (in $\mathrm{g} \mathrm{mol}^{-1}$ ), $\Re$ is the gas constant (in erg $\mathrm{K}^{-1} \mathrm{~mol}^{-1}$ ), and

$$
\wp_{\text {subl }}(T)=\Lambda \exp \left[-\frac{L}{\Re T}\right],
$$

with $\Lambda$ and $L$, the latent heat of sublimation, being constants.

The position at time $t$ of a particle, moving in a concave hyperbolic orbit of the perihelion distance $q$ and eccentricity $e$, is given by a hyperbolic eccentric anomaly $f$, which is related to $t$ by

$$
e \sinh f-f=\frac{k_{\text {grav }}(1-\beta)^{\frac{1}{2}}(e-1)^{\frac{3}{2}}}{q^{\frac{3}{2}}}\left(t-t_{\pi}^{*}\right),
$$

where $k_{\text {grav }}$ is the Gaussian gravitational constant, $\beta<1$ is again the radiation-pressure parameter from Eq. (1), and $t_{\pi}^{*}$ is the time of the particle's passage through perihelion. The anomaly $f$ can for any $t-t_{\pi}^{*}$ be computed by successive iterations of Eq. (24). If $t$ is reckoned from $t_{\pi}$, the time of the comet's passage through perihelion, then in Eq. (24) one substitutes $t-t_{\pi}^{*}=t-t_{\pi}-\left(t_{\pi}^{*}-t_{\pi}\right)$. Since the heliocentric distance at time $t$ is equal to

$$
r(t)=\frac{q}{e-1}(e \sinh f-1),
$$

we can insert quantities from Eqs. (21) to (25) into Eq. (20) to obtain the integral in the form

$$
\int_{t_{\mathrm{rel}}}^{t_{\mathrm{obs}}} \dot{a}_{\mathrm{subl}} d t=\Psi \int_{f_{\mathrm{rel}}}^{f_{\mathrm{obs}}} T^{-\frac{1}{2}} \exp \left[-\frac{L}{\Re T}\right] r d f,
$$

where $f_{\text {rel }}$ and $f_{\text {obs }}$ are the hyperbolic anomalies at times $t_{\mathrm{rel}}$ and $t_{\mathrm{obs}}$, and

$$
\Psi=\frac{\Lambda \mu^{\frac{1}{2}}}{\rho} \frac{q^{\frac{1}{2}}}{k_{\text {grav }}}[2 \pi \Re(1-\beta)(e-1)]^{-\frac{1}{2}} .
$$

For a given $t_{\text {obs }}$, the time of release $t_{\text {rel }}$ and the radiation-pressure parameter $\beta$ are determined by fitting the imaged tail's morphology with a set of syndynames that describe the distribution of dust particles in the tail. These computations automatically provide the particles' perihelion distance $q$ and eccentricity $e$. Now the magnitude of the integrated sublimation effect depends on four 
quantities - the sublimation-pressure constant $\Lambda$ (related to the entropy of the particle material), the latent heat (or enthalpy) of sublimation $L$, the molar weight $\mu$, and the bulk density $\rho$. The effect is also a function of the variations in the equilibrium temperature $T$ along the particles' hyperbolic orbits. In practice, the computations are difficult because this temperature is a complicated function of the optical, thermophysical, and morphological properties of the particle material, all of which vary with time and heliocentric distance.

We limit ourselves to a brief examination of the likelihood that the dust of $\mathrm{C} / 2011 \mathrm{~W} 3$ that begins to sublimate profusely at $\sim 1.8 R_{\odot}$ consists of olivine-dominated silicate particles released from the nucleus, respectively, 0.08 and 0.12 day before perihelion and subjected to solar radiation pressure of $\beta=0.6$. The first, more conservative case implies that $q=1.7294 R_{\odot}, e=2.4513$, and $t_{\pi}^{*}-t_{\pi}=-0.0008$ day, whereas the other case leads to $q=1.8841 R_{\odot}, e=2.1678$, and $t_{\pi}^{*}-t_{\pi}=+0.0078$ day. For the temperature we use only a fairly crude approximation based on a black-body temperature and an assumed thermal radiative regime,

$$
T(r)=\frac{280 \mathrm{~K}}{r^{\frac{1}{2}}}
$$

The sublimation rate and other properties of olivine depend on the ratio of magnesium to iron in its formula $\mathrm{Mg}_{z} \mathrm{Fe}_{2-z}^{2+} \mathrm{SiO}_{4}$, where $0 \leq z \leq 2$. Following Barthelmy (2010), we adopt $z=1.6$ for the empirical formula, $\mu=153.31 \mathrm{~g} \mathrm{~mol}^{-1}$ for the molar weight, and $\rho=3.32$ $\mathrm{g} \mathrm{cm}^{-3}$, an average mineralogical density, for the bulk density of submicron-sized olivine-dominated grains.

We now require that the integrated sublimation effect computed from Eq. (26) amounts to $0.2 \mu \mathrm{m}$ in the particle radius, the average size of silicate grains near $\beta=0.6$ (e.g., Kimura, Ishimoto, \& Mukai 1997; Kimura et al. 2002) and search for the relationship between the two remaining unknowns - the sublimation-pressure constant $\Lambda$ and the heat of sublimation $L$ - that satisfy this total loss in the particle radius between $t_{\text {rel }}$ and $t_{\text {obs }}$. The two values of $t_{\text {rel }}$ thus lead to two sets of solutions, which are insensitive to the observation time, as long as the observation takes place after the particle's passage through perihelion. The solutions are presented in Fig. 11 in a range of 100 to $150 \mathrm{kcal} \mathrm{mol}^{-1}$ for the heat of sublimation and compared with several sources of information on sublimation of silicates and other potentially relevant materials. Because Eqs. (26) and (27) show that the integrated sublimation effect depends on $\Lambda$ only through a function $\Lambda \mu^{\frac{1}{2}} \rho^{-1}$, it is this function that is plotted in Fig. 11 against the heat of sublimation $L$.

Nagahara, Mysen, \& Kushiro (1994) published the sublimation-pressure constants for the two endmembers of the olivine solid solution system. Including the molar weight and density, the data are as follows: $\Lambda=6.72 \times 10^{13} \mathrm{~Pa}, L=129.74 \mathrm{kcal} \mathrm{mol}^{-1}, \mu=140.69$ $\mathrm{g} \mathrm{mol}{ }^{-1}$, and $\rho=3.27 \mathrm{~g} \mathrm{~cm}^{-3}$ for forsterite $(\mathrm{Mg}$ endmember, for which $z=2$ ); and $\Lambda=2.48 \times 10^{15} \mathrm{~Pa}$, $L=119.94 \mathrm{kcal} \mathrm{mol}^{-1}, \mu=203.78 \mathrm{~g} \mathrm{~mol}^{-1}$, and $\rho=4.39$ $\mathrm{g} \mathrm{cm}^{-3}$ for fayalite (Fe-endmember, for which $z=0$ ). Because the sublimation properties vary smoothly between the two endmembers (e.g., Gail \& Sedlmayr 1999), we plot in Fig. 11 the dependence of $L$ on $\Lambda \mu^{\frac{1}{2}} \rho^{-1}$ for the entire set of olivine members. In their study, Kimura et al. (2002) used Nagahara, Mysen, \& Kushiro's (1994) sublimation parameters for forsterite, but the molar weight for olivine with $z=1.1$, and the density of $3.3 \mathrm{~g} \mathrm{~cm}^{-3}$, so that in Fig. 11 their point would be located just 0.04 in $\log \left(\Lambda \mu^{\frac{1}{2}} \rho^{-1}\right)$ to the right of forsterite. For reference and orientation we also plot in the figure: (i) the data point for "silicates", as used by Kimura, Ishimoto, \& Mukai (1997) $-\Lambda=1.07 \times 10^{13} \mathrm{~Pa}, L=113.98 \mathrm{kcal} \mathrm{mol}^{-1}$, $\mu=67.0 \mathrm{~g} \mathrm{~mol}^{-1}$, and $\rho=2.37 \mathrm{~g} \mathrm{~cm}^{-3}$; (ii) the numbers that the most recent CRC Handbook for Chemistry and Physics (Haynes 2011) provides for quartz $\left(\mathrm{SiO}_{2}\right)$ : $\Lambda=1.38 \times 10^{13} \mathrm{~Pa}, L=134.55 \mathrm{kcal} \mathrm{mol}^{-1}, \mu=60.08 \mathrm{~g}$ $\mathrm{mol}^{-1}$, and $\rho=2.65 \mathrm{~g} \mathrm{~cm}^{-3}$; and (iii) an extrapolated relationship between $\Lambda$ and $L$, as used by Sekanina (2003) for some metallic elements in his study of the light curves of SOHO sungrazers, with an average value of 4 for the ratio $\sqrt{\mu} / \rho$, which varied roughly between 1 and 7 .

To the extent that we can - despite the approximations - express some confidence from the meaning of the plot in Fig. 11, we conclude that silicates dominated by Mg-rich olivine could represent a plausible candidate for the material that made up dust particles that began to sublimate profusely in the tail of comet $\mathrm{C} / 2011 \mathrm{~W} 3$ near a heliocentric distance of $1.8 R_{\odot}$.

An effect that has not been accounted for in this paper is the deviation of the radiation-pressure acceleration from the $r^{-2}$ dependence very close to the Sun, as briefly mentioned in Sec. 6. The reason for this deviation is twofold: (i) very close to the Sun, the particle is subjected to the radiation coming only from that part of the photosphere that is above the particle's horizon, and (ii) the photosphere is closer to the particle by up to 1 $R_{\odot}$ than the Sun's center. The first fact decreases the magnitude of the radiation pressure relative to that from the standard inverse-square power law, while the latter one increases it. Combined, the second influence prevails, and the integration over the relevant region shows that at a distance $r$ from the Sun's center the actual acceleration, $\beta^{*}$, exceeds the standard value, $\beta$, by

$$
\frac{\beta^{*}}{\beta}=2\left(\frac{r}{R_{\odot}}\right)^{2}\left[1-\sqrt{1-\left(\frac{R_{\odot}}{r}\right)^{2}}\right] .
$$

It is clear that $\beta^{*}=\beta$ for large values of the ratio $r / R_{\odot}$, while $\beta^{*} / \beta$ is equal to 1.01 for $r=5 R_{\odot}, 1.15$ for $r=1.5 R_{\odot}$, and 1.29 for $r=1.2 R_{\odot}$. For the borderline heliocentric distances involved in the sublimation problem this effect does not exceed 10 percent and is not significant enough to invalidate the results.

\section{RAPID BUILDUP OF THERMAL STRESS IN NUCLEUS' INTERIOR AND PROCESS OF CATACLYSMIC FRAGMENTATION}

From the information presented, the process of disintegration of comet $\mathrm{C} / 2011 \mathrm{~W} 3$ appears to have been followed for at least four days immediately after perihelion. A drop in activity around December 15.7 UT, or 0.3 day before perihelion, may have been related to sublimation of dust (Sec. 11) and was not necessarily an early signature of the process of disintegration. On the other hand, the sudden and permanent loss of the nuclear 


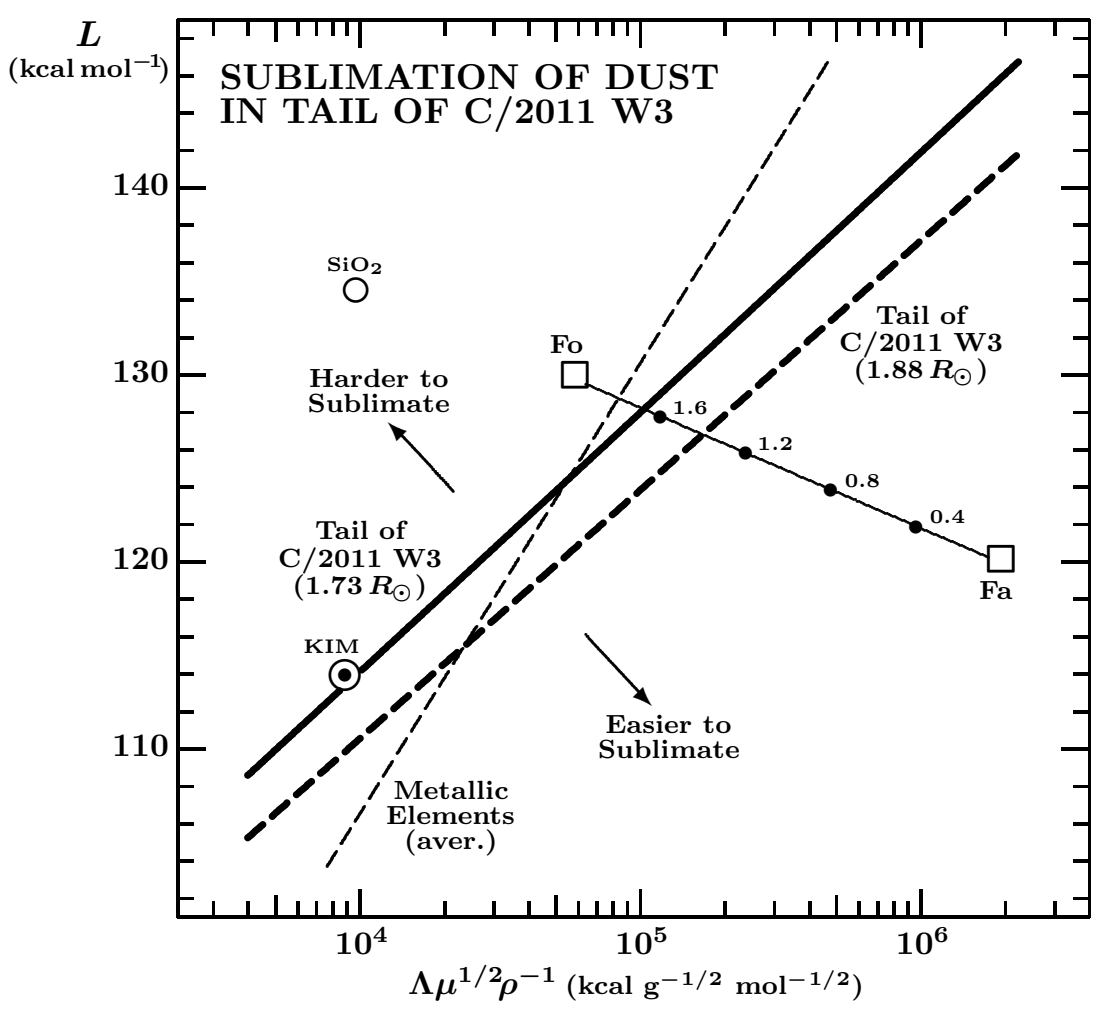

Figure 11. The relationship between the parameter $\Lambda \mu^{\frac{1}{2}} \rho^{-1}$ - a function of the sublimation-pressure constant, molar weight, and bulk density - and the latent heat of sublimation $L$. The uninterrupted thick curve is the set of solutions that refer to the onset of profuse sublimation of dust in the tail of comet $\mathrm{C} / 2011 \mathrm{~W} 3$ at $1.73 R_{\odot}$ from the Sun. The dashed thick curve is the set of solutions that refer to the onset of profuse sublimation at $1.88 R_{\odot}$. The two squares are the locations in the plot of the two endmembers of the olivine solid solution system, based on the sublimation-pressure constants by Nagahara, Mysen, \& Kushiro (1994): forsterite (Fo; Mg-endmember) and fayalite (Fa; Fe-endmember). The dots on the Fo-Fa connecting line refer, from left to right, to the members of the olivine system, whose formula is, respectively, $\mathrm{Mg}_{1.6} \mathrm{Fe}_{0.4} \mathrm{SiO}_{4}, \mathrm{Mg}_{1.2} \mathrm{Fe}_{0.8} \mathrm{SiO}_{4}, \mathrm{Mg}_{0.8} \mathrm{Fe}_{1.2} \mathrm{SiO}_{4}$, and $\mathrm{Mg}_{0.4} \mathrm{Fe}_{1.6} \mathrm{SiO}_{4}$. For reference and orientation, we also

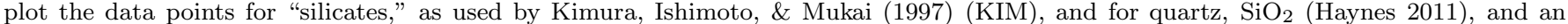
extrapolated relationship between $\Lambda$ and $L$, as used by Sekanina (2003) for some metallic elements in his study of the light curves of SOHO sungrazers, with an average value of 4 for the ratio $\sqrt{\mu} / \rho$. The constants for olivine used by Kimura et al. (2002) are not shown, as they nearly coincide with those for forsterite.

condensation observed first between December 19.4 and 20.3 UT (Fig. 1) was clear evidence of terminal collapse. There has been no trace of new activity detected from December 20 on, indicating that the comet's progressive fading after this date only reflected the rate of dispersal in space of the dust ejecta released during the December 16-20 period of activity.

It is not absolutely clear whether in this critical period of time the activity proceeded more or less continuously or was dominated by discrete outbursts other than the terminal event(s). Either way, this activity must account for the formation of the quasi-parabolic envelope, encircling the spine tail, and also visible in its developing phase in the images from December 17 through 19. Although the particle-ejection velocities in the spine tail were much lower than in the envelope (Sec. 8), the outburst that triggered the formation of the spine tail was obviously the most devastating for the comet in that it was associated with the cataclysmic fragmentation of the entire residual mass of the nucleus. The low velocities of mostly large-sized debris may explain why to an earthbased observer the process appeared to have taken at least another two days to complete. Simple calculations support this scenario. Indeed, released on December 17.6 UT and expanding at $\sim 20 \mathrm{~m} \mathrm{~s}^{-1}$, large dust in the coma would occupy a volume of about $6000 \mathrm{~km}$, or $10^{\prime \prime}$, in diameter on December 19.4 UT, when the comet still displayed a nuclear condensation (Fig. 1), and nearly $10,000 \mathrm{~km}$, or $17^{\prime \prime}$, in diameter on December $20.3 \mathrm{UT}$, when the condensation was already gone. The diameter of the bright condensation in the Malargue image from December 19 is in fact about $30^{\prime \prime}$, which suggests a major contribution of dust from the previous activity, while the breadth of the nascent spine tail in the Malargue image from December 20 is just about the expected $17^{\prime \prime}$. The bright streamer in the December 19 image - the early appearance of the spine tail - can be traced to a distance of at least $6^{\prime}$ from the nucleus; its end point indicates the radiation-pressure acceleration parameter of $\beta \simeq 0.12$, that is, the presence of dust particles larger than $10 \mu \mathrm{m}$ in diameter, when released during the December 17.6 outburst.

It is important to realize that the timing of this event offers unequivocal evidence of a gradual deterioration of the comet's health and thereby demonstrates its appreciable resistance to the hostile environment in close proximity of the Sun. This conclusion clearly shows that the nucleus could not possibly have been held together merely by self-gravity. The comet's significantly delayed response does not favor models that describe the 
cometary nucleus as a strengthless or very poorly cemented rubble-pile structure, which - by virtue of being unconsolidated debris - reacts to tidal forces promptly (e.g., Asphaug \& Benz 1994, 1996; Solem 1994). Evidence from comet $\mathrm{C} / 2011 \mathrm{~W} 3$ thus leads to the same conclusion as evidence from the close-up imaging of the nuclei of $81 \mathrm{P} /$ Wild and $9 \mathrm{P} /$ Tempel, which were independently found incompatible with the rubble pile model by, respectively, Brownlee et al. (2004) and Thomas et al. (2007).

In a very recent study, Gundlach et al. (2012) explain the survival of sungrazing comets like $\mathrm{C} / 2011 \mathrm{~W} 3$ within the Roche limit of the Sun by the counterpressure due to virtually isotropic outgassing from the nucleus, which more than compensates for the tidal disruption force. For C/2011 W3 such a scenario is, however, unnecessary, because its nuclear diameter at perihelion was almost certainly less than $1 \mathrm{~km}$ and the tidal stress therefore less than $\sim 10 \mathrm{~Pa}$ (as seen from Fig. 2 in Gundlach et al.'s paper), a benign effect for any comet except an essentially strengthless one. A major problem with Gundlach et al.'s scenario is that it is inconsistent with the extensively observed duplicity of the sungrazing comet C/1965 S1 (e.g., Pohn 1965, Thackeray 1965, Iannini 1966, Lourens 1966), whose estimated diameter of less than $9 \mathrm{~km}$ (Knight et al. 2010) and perihelion distance of $1.67 R_{\odot}$ place the comet deep inside Gundlach et al.'s "safe" zone.

What property was it then that made C/2011 W3 survive perihelion on the one hand but suddenly disintegrate nearly 2 days later on the other hand? Always suspected in any substantially delayed response are the thermal effects in the comet's nuclear interior, as transport of heat takes time. The resulting temperature changes, dramatically enhanced in the sungrazers near perihelion, impose a severe burden in the form of thermal stress, which may not only dwarf the tidal stress but could last for extended periods of time.

To investigate heat transport effects in the nucleus of $\mathrm{C} / 2011 \mathrm{~W} 3$, we have followed the procedure outlined and applied by Sekanina (2009) to study the giant explosions of $17 \mathrm{P} /$ Holmes. The approach assumes that the solar radiation impinging on the nucleus is spent - in rather extensive bare areas of the surface, devoid of ice - only on thermal reradiation and conduction of heat into the interior. This case is therefore, in principle, contrary to that considered by Gundlach et al. (2012). Being interested merely in basic, order-of-magnitude information on thermal stress, we have applied only the standard (isothermal) version of the one-dimensional heat-transfer problem in a spherical object. We have assumed that an initial central temperature of the nucleus is $60 \mathrm{~K}$ and a coefficient of effective thermal conductivity $K_{\text {eff }}=0.2$ $\mathrm{W} \mathrm{m}^{-1} \mathrm{~K}^{-1}$, which was found the most likely value to fit the recurrence of the giant explosions of comet $17 \mathrm{P}$ once every 115 years or so (Sekanina 2009) and which is also well within the interval compatible with the range of the thermal inertia that Davidsson, Gutiérrez, \& Rickman (2009) derived in their analysis of near-infrared thermal emission spectra of features on the nucleus of comet 9P/Tempel. With the numbers used for the specific heat and the bulk density, this value of $K_{\text {eff }}$ is equivalent to an effective thermal diffusivity of $\kappa_{\mathrm{eff}} \simeq 0.006 \mathrm{~cm}^{2} \mathrm{~s}^{-1}$.

The results of applying the heat-transfer equation to the nucleus of comet $\mathrm{C} / 2011 \mathrm{~W} 3$ are presented in Figs.
12 and 13. Figure 12, a plot of temperature against depth beneath the surface, exhibits an enormous perihelion asymmetry. The plot shows for example that the same temperature reached 0.6 day before perihelion at a depth of less than 10 meters is reached 1.6 days after perihelion at a depth of more than 40 meters. A significant conclusion from Fig. 13, a plot of the postperihelion temperature with heliocentric distance, is that except in the topmost 15-meter-deep layer, the temperature keeps increasing as the comet recedes from the Sun; it is still increasing at large heliocentric distances. In this regime, the effects of thermal stress must keep increasing and must further be enhanced by sudden activity from scattered reservoirs or "pockets" of highly volatile substances, such as amorphous water ice, reached by the rapidly penetrating thermal wave deep inside the nucleus. Sooner or later, the combination of thermal stress and such explosive events is bound to have catastrophic consequences for the nucleus. We thereby find an entirely plausible mechanism for cascading fragmentation of sungrazers, large or small, well away from the Sun.

The general equations for the radial and tangential components of thermal stress, $\sigma_{\|}, \sigma_{\perp}$, at distance $h$ from the center of a sphere of radius $h_{\star}$ are given by (Timoshenko \& Goodier 1970)

$$
\begin{aligned}
& \sigma_{\|}(h)=2 \chi\left[\frac{\Phi\left(h_{\star}\right)}{h_{\star}^{3}}-\frac{\Phi(h)}{h^{3}}\right], \\
& \sigma_{\perp}(h)=\chi\left[\frac{2 \Phi\left(h_{\star}\right)}{h_{\star}^{3}}+\frac{\Phi(h)}{h^{3}}-T(h)\right],
\end{aligned}
$$

where

$$
\chi=\frac{\alpha_{\mathrm{L}} E_{\mathrm{Y}}}{1-\nu}
$$

is the thermal stress parameter and

$$
\Phi(h)=\int_{0}^{h} T(s) s^{2} d s,
$$

with $T(s)$ being the temperature at distance $s$ from the center. The object's thermal and mechanical properties determine the coefficient of linear thermal expansion $\alpha_{\mathrm{L}}$, Young's modulus $E_{\mathrm{Y}}$, and Poisson's ratio $\nu$. From the first of Eqs. (30) we note that at the surface $\left(h=h_{\star}\right)$ the radial component $\sigma_{\|}$of thermal stress is always nil. On the other hand, at the center the radial and tangential components have the same magnitude equal to

$$
\lim _{h \rightarrow 0} \sigma_{\|}=\lim _{h \rightarrow 0} \sigma_{\perp}=2 \chi\left[\frac{\Phi\left(h_{\star}\right)}{h_{\star}^{3}}-\frac{1}{3} T_{0}\right],
$$

where $T_{0}=T(0)$ is the central temperature. In addition, there exists a mean interior temperature $\langle T\rangle$ such that

$$
\int_{0}^{h \star} T(s) s^{2} d s=\langle T\rangle \int_{0}^{h \star} s^{2} d s=\frac{1}{3}\langle T\rangle h_{\star}^{3},
$$

so that

$$
\lim _{h \rightarrow 0} \sigma_{\|}=\lim _{h \rightarrow 0} \sigma_{\perp}=\frac{2}{3} \chi\left[\langle T\rangle-T_{0}\right] .
$$

As the distribution of temperature in the nucleus' interior keeps steadily increasing with time and heliocentric distance following the perihelion passage, so do both $\langle T\rangle$ 


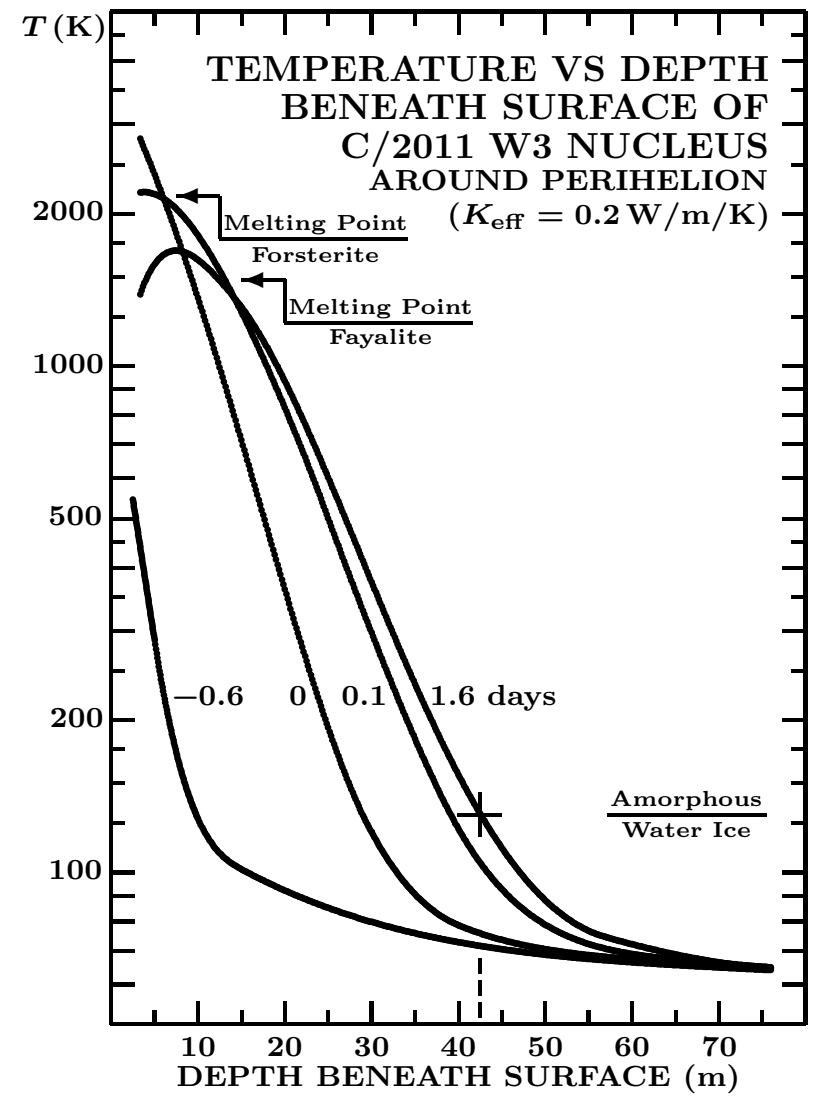

Figure 12. Temperature variations with depth beneath the surface derived for the nucleus of comet C/2011 W3 with a maximum diameter of 600 meters. The one-dimensional heat-transfer equation has been solved, averaging the incident solar energy over the surface, assuming that the energy is spent only on the conduction into the nucleus and thermal reradiation (bare surface, devoid of ices or other substances that would sublimate), and adopting a Bond albedo of 0.03 , an emissivity of unity, a coefficient of effective thermal conductivity of $2 \times 10^{4} \mathrm{erg} \mathrm{cm}^{-1} \mathrm{~s}^{-1} \mathrm{~K}^{-1}$, a specific heat capacity of $8 \times 10^{6} \mathrm{erg} \mathrm{g}^{-1} \mathrm{~K}^{-1}$, and a bulk density of $0.4 \mathrm{~g} \mathrm{~cm}^{-3}$. The individual curves are identified by the time from perihelion: 0.6 day before perihelion, at perihelion, and 0.1 and 1.6 days after perihelion, when comet $\mathrm{C} / 2011 \mathrm{~W} 3$ was at heliocentric distances of, respectively, $0.0731,0.00555,0.0196$, and 0.144 AU. Also marked are the crystallization temperature of amorphous water ice and the melting points of forsterite and fayalite.

(much more so than $T_{0}$ ) and the thermal stress in the center. The tendency toward a sungrazer's breakup far from the Sun due to thermal forces is confirmed even without the existence of subsurface reservoirs of highly volatile ices.

In Eqs. (30) we have divided the entire range of distances from the center, $0 \leq h \leq h_{\star}$, into $n$ separate intervals $h_{i-1} \leq h \leq h_{i}(i=1, \ldots, n)$, where $h_{0}=0$ and $h_{n}=h_{\star}$, and fitted the temperature $T$ from Fig. 12 inside each interval by a polynomial of power $m$,

$$
T(h)=\sum_{k=0}^{m} a_{k, i} h^{k}, \quad i=1, \ldots, n .
$$

The integral $\Phi\left(h_{\star}\right)$ is then given by

$$
\Phi\left(h_{\star}\right)=\sum_{i=1}^{n} \sum_{k=0}^{m} \frac{a_{k, i}}{k+3}\left(h_{i}^{k+3}-h_{i-1}^{k+3}\right)
$$

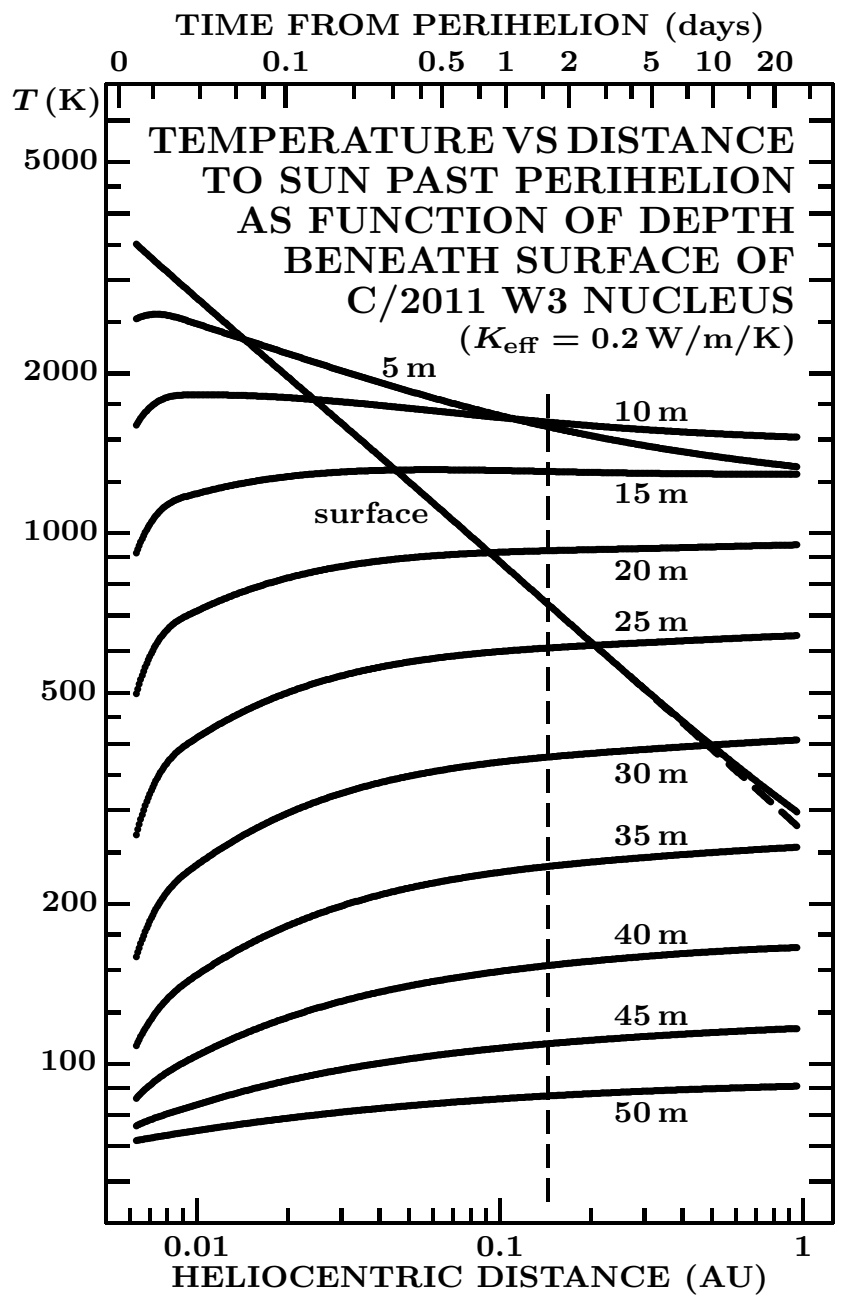

Figure 13. Temperature variations with heliocentric distance at the surface and at 10 depths beneath the surface derived for the nucleus of comet $\mathrm{C} / 2011 \mathrm{~W} 3$. The computations are based on the model and assumptions described in the caption to Fig. 12. The surface-temperature variations are shown for preperihelion (broken) and postperihelion branches of the orbit, all other curves are postperihelion. The broken line parallel to the axis of ordinates shows the heliocentric distance $0.144 \mathrm{AU}$, at which the cataclysmic fragmentation event occurred on December 17.6 UT.

and for distance $h$ from the center in a $(j+1)$-st interval, $h_{j} \leq h \leq h_{j+1}(0 \leq j \leq n-1)$, the integral $\Phi(h)$ is equal to

$$
\begin{aligned}
\Phi(h)= & \sum_{i=1}^{j} \sum_{k=0}^{m} \frac{a_{k, i}}{k+3}\left(h_{i}^{k+3}-h_{i-1}^{k+3}\right) \\
& +\sum_{k=0}^{m} \frac{a_{k, j+1}}{k+3}\left(h^{k+3}-h_{j}^{k+3}\right) .
\end{aligned}
$$

Because Fig. 12 shows that the temperature changes very little at depths exceeding $60-70$ meters, the described approach can also be used to approximate the magnitude and distribution of thermal stress in the interior of the nucleus of smaller dimensions, whose center is assumed to be at depth $h_{\star}-\hbar$ rather than at $h_{\star}$. Reckoning distance $h$ (but not the interval boundaries $h_{i-1}$ and $h_{i}$ ) from the new center at this depth, we can write Eq. (36) 
in the form

$$
T(h)=\sum_{k=0}^{m} a_{k, i}(h+\hbar)^{k}=\sum_{k=0}^{m} b_{k, i} h^{k}, \quad i=j, \ldots, n,
$$

when $\hbar$ is in an interval $h_{j-1} \leq \hbar \leq h_{j}(1 \leq j \leq n)$, and for $0 \leq k \leq m$

$$
b_{k, i}=\sum_{l=k}^{m}\left(\begin{array}{l}
l \\
k
\end{array}\right) a_{l, i} \hbar^{l-k}, \quad i=j, \ldots, n .
$$

Coefficients $b_{k, i}$ now replace $a_{k, i}$ in Eqs. (37) and (38), in which the interval boundaries have to be modified accordingly.

This set of formulas can also be used when the size of the nucleus is truncated to $h_{\bullet}<h_{\star}$ on the outside, that is, when it is desirable to eliminate the contribution to thermal stress from a surface layer once it has been removed by erosion or otherwise destroyed. In this case the integral $\Phi\left(h_{\bullet}\right)$ is computed from Eq. (38) rather than (37), either with the coefficients $a_{k, i}$ or $b_{k, i}$, depending on whether $\hbar$ is or is not nil.

To allow for a gradual erosion of the surface layer in the proximity of the Sun, the internal-temperature distribution presented in Figs. 12 and 13 has been used to compute the thermal-stress profiles for the nucleus 400 meters in diameter at 0.6 day before perihelion, 280 meters in diameter at perihelion, and 150 meters in diameter 1.6 days after perihelion. The results are presented in two steps. In the first step, the relative variations in the radial and tangential components of thermal stress, normalized to its magnitude at the center of the nucleus at perihelion, are displayed against depth beneath the surface in Fig. 14. The major properties of the stress curves are as follows:

(1) Just as with temperature, thermal stress continues to climb even as the comet recedes from the Sun; measured by its magnitude at the nucleus' center, the thermal stress at perihelion is about 16 times higher than 0.6 day before perihelion, but 2.4 times lower than 1.6 days after perihelion.

(2) At a given time, the radial component of thermal stress is always tension, increasing from zero at the surface, as already noted; the tangential component reaches its maximum at the surface as compression, but changes to tension a few tens of meters beneath the surface, increasing toward the center.

(3) Both components reach maximum tension at the center of the nucleus, where they have the same magnitude, as implied by Eq. (33).

(4) Cracks, whose formation is triggered by increasing thermal stress at large depths due to the thermal wave's gradual penetration into the interior, are bound to open preexisting subsurface reservoirs of highly volatile ices (such as amorphous water ice), initiating their explosion when heated up to the activation temperature.

(5) The steady increase in thermal stress at the center of the nucleus over long periods of time after perihelion is likely to be a driving force behind the episodes of seemingly spontaneous cascading fragmentation at large heliocentric distances, which account for the SOHO sungrazers' observed scatter in elements other than orbital period.

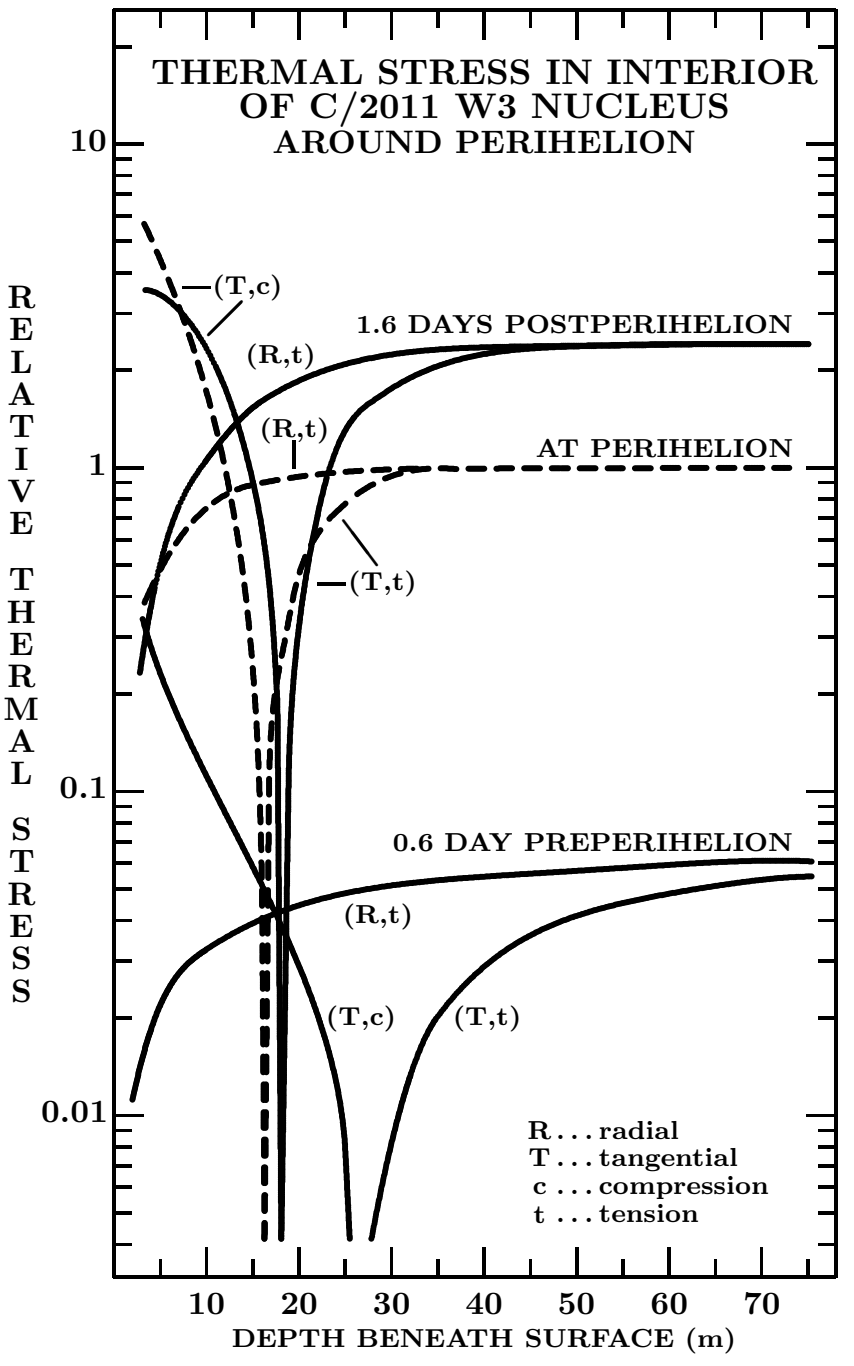

Figure 14. Relative thermal stress in the interior of comet C/2011 W3 as a function of depth beneath the surface. Derived from the temperature variations 0.6 day before perihelion for the nucleus 400 meters in diameter (solid), at perihelion for 280 meters in diameter (broken), and 1.6 days after perihelion for 150 meters in diameter (solid), the stress curves are normalized to thermal stress at the nucleus' center at perihelion. The segments of the curves are marked to distinguish between the radial and tangential stress components and whether the stress was a compression or tension.

Measured in absolute units, the magnitude of thermal stress depends on the stress parameter $\chi$, given by Eq. (31) and consisting of three physical quantities: Young's modulus $E_{\mathrm{Y}}$, the coefficient of linear thermal expansion $\alpha_{\mathrm{L}}$, and Poisson's ratio $\nu$. Since their values for cometary material are unknown, we make in the following an effort to constrain each of them to our best ability by using meteoritic, lunar, and terrestrial analogues. Given our conclusion in Sec. 11 on the nature of dust sublimating from comet C/2011 W3 near the Sun, a plausible candidate among the terrestrial samples appears to be an olivine-based silicate material.

Young's modulus. There is a large number of papers with information on Young's modulus, but they mostly refer to samples of zero or near-zero porosity. For the Moon, Pritchard \& Stevenson (2000) estimated that the effective Young's modulus in the outer layers of the lu- 
nar lithosphere is between 5 and $10 \mathrm{GPa}$. For ordinary chondrites the topic was recently updated by Kimberley \& Ramesh (2011). They also provided the results of their experiments aimed at determining the elastic and mechanical properties of a particular L5 ordinary chondrite found in Antarctica, showed that the outcome depends on whether the data were obtained under quasistatic or dynamic conditions, compared their results to the findings from the numerous studies of the atmospheric breakup of meteors, and emphasized that the propagation of cracks is gradual because the crack speeds are much lower than the limit defined by elastodynamic fracture theory. Although Kimberley \& Ramesh did not consider thermal stress, their conclusions are relevant, because they found that the value of the Young's modulus for a particular sample with a 13 percent porosity depended on a strain rate and range of strain; at slow rates and strains of up to $0.01, E_{\mathrm{Y}}=3.2 \mathrm{GPa}$, at strains ranging from 0.01 to 0.03 and much higher strain rates (appropriate in hypervelocity impact phenomena), $E_{\mathrm{Y}}=8.5 \mathrm{GPa}$; this behavior has also been observed in some brittle terrestrial materials, e.g., ceramics (Wang \& Ramesh 2004). Pertinent to our problem is the lower value of $E_{\mathrm{Y}}$.

From analysis of limited samples of meteorites, rarely including carbonaceous chondrites (e.g., Flynn, Klöck, \& Krompholz 1999), most values found for Young's modulus were one order of magnitude higher than the quoted result by Kimberley \& Ramesh, but their porosities were mostly lower than 13 percent. A key work was published by Yomogida \& Matsui (1983) on the mechanical, thermal, and elastic properties (measured quasistatically) of 20 ordinary chondrites of $\mathrm{H}$ and $\mathrm{L}$ types. For some of these properties the authors compared the meteorites with lunar samples studied by others. One of Yomogida \& Matsui's findings was the strong dependence of the elastic properties on porosity. They showed that even a low porosity reduces the values of the elastic parameters, including Young's modulus, much more for the meteorites and the lunar samples than for terrestrial rocks. While there are fairly large differences between the individual samples, the steep drop in Young's modulus $E_{\mathrm{Y}}(\psi)$ with porosity $\psi$ is for 19 out of the 20 chondrites fitted by

$$
E_{\mathrm{Y}}(\psi)=\text { const } \cdot \exp \left[-U\left(\frac{\psi}{1-\psi}\right)^{\frac{1}{4}}\right]\left(\text { for } \psi \geq \psi_{0}\right)
$$

where $\quad U=13.8 \pm 1.2$ and const $=85_{-42}^{+82} \times 10^{12} \mathrm{~Pa}$ $\left(\psi_{0}=0.05\right)$ for 13 samples (high $E_{\mathrm{Y}}$ group) and $31_{-10}^{+15} \times 10^{12} \mathrm{~Pa}\left(\psi_{0}=0.02\right)$ for 6 samples (low $E_{\mathrm{Y}}$ group). This expression provides a substantially better fit than Warren's (1969) formula used by Yomogida \& Matsui (1983) in their Fig. 8 to match a normalized elastic modulus of both the meteorites and the lunar samples. Although by 2001 the porosity (with values of up to 30 percent) had already been known for more than 450 stony meteorites (Britt \& Consolmagno 2003), information on their elastic moduli seems to be lagging behind.

A variety of studies of terrestrial samples provides constraints on Young's modulus of olivine. However, many measurements refer to temperatures in excess of $1000 \mathrm{~K}$.
For example, Tait (1992) used a value of $197 \mathrm{GPa}$ at $1470 \mathrm{~K}$, while Hiraga, Anderson, \& Kohlstedt (1993) gave values of 159,155 , and $154 \mathrm{GPa}$ at, respectively, $1373 \mathrm{~K}$, $1473 \mathrm{~K}$, and $1523 \mathrm{~K}$. Chung (1970) measured the bulk modulus and Poisson's ratio of olivine as a function of the $\mathrm{Mg} / \mathrm{Fe}$ ratio at room temperature $(296 \mathrm{~K})$ and zero pressure. In terms of Young's modulus, his results show a systematic trend from a maximum of $198 \mathrm{GPa}$ for forsterite to a minimum of $141 \mathrm{GPa}$ for fayalite. An extensive study of the dependence on the temperature of the elastic properties of a single crystal of forsterite was published by Suzuki, Anderson, \& Sumino (1983), who found that the elastic moduli increase with decreasing temperature at a rate of about 1.3 percent per $100 \mathrm{~K}$ between $0 \mathrm{~K}$ and $1200 \mathrm{~K}$. From their values of the bulk modulus and Poisson's ratio, Young's modulus of forsterite comes out to be $175 \mathrm{GPa}$ at $1500 \mathrm{~K}$ and $207 \mathrm{GPa}$ at $100 \mathrm{~K}$, an increase of 18 percent. All these numbers are fairly consistent with the highest values of Young's modulus near 140 GPa measured by Yomogida \& Matsui (1983) for the meteorites of low porosity. It therefore appears that the temperature effect is insignificant relative to the porosity effect. A detailed investigation of Young's modulus variations with porosity for a variety of terrestrial materials was published by Phani \& Sanyal (2005), who determined that for colloidal gel derived silica the relative modulus varied as

$$
\frac{E_{\mathrm{Y}}(\psi)}{\left(E_{\mathrm{Y}}\right)_{0}}=\frac{(1-\psi)^{2}}{1+\frac{3}{2} \psi}
$$

for porosities smaller than 0.85 . The slope of the curve is somewhat similar to that shown by Yomogida \& Matsui (1983) for a sintered material and tuff in their Fig. 8; it is considerably less steep than the slope for the meteorites and the lunar samples.

Coefficient of linear thermal expansion. This coefficient is on a recently compiled "wish" list of 12 physical quantities of meteorites, the data on which are desired most by the scientific community (Consolmagno, Britt, \& Opeil 2010), a fact that cogently illustrates the urgent need for this practically nonexistent information.

It has been known for nearly a century that the thermal expansion coefficient is generally temperature dependent and that its rate of variation in crystallic solids is, at least at relatively low temperatures, approximately proportional to that of the specific heat capacity at constant volume (e.g., Austin 1952). Given that our primary interest is the temperature range from $\sim 70 \mathrm{~K}$ to $\sim 300 \mathrm{~K}$, we have focused on a search for relevant sets of data on olivine-based silicates that should be as consistent with this temperature interval as possible. Once available, the data on the coefficient of linear thermal expansion have been fitted to satisfy a properly scaled formula for the Einstein crystal model (e.g., Rogers 2005),

$$
\alpha_{\mathrm{L}}(T)=\lambda\left(\frac{\Omega}{T}\right)^{2}\left[\sinh \left(\frac{\Omega}{T}\right)\right]^{-2},
$$

where $\lambda$ and $\Omega$ are constants to be determined from the data. (The known fact that this equation does not predict correct values of heat capacity at low temperatures is irrelevant to our using it to fit a set of $\alpha_{\mathrm{L}}$ values.)

Samples of a hot-pressed olivine aggregate were used by Aizawa, Ito, \& Tatsumi (2001) to measure its vol- 
umetric coefficient of thermal expansion, $\alpha_{\mathrm{V}}$, but the temperature range was from $300 \mathrm{~K}$ up, which makes the data, requiring much extrapolation, only marginally useful. After conversion to $\alpha_{\mathrm{L}}=\frac{1}{3} \alpha_{\mathrm{V}}$, we found that fitting the data between $300 \mathrm{~K}$ and $600 \mathrm{~K}$ by Eq. (43) requires $\lambda=12.0 \times 10^{-6} \mathrm{~K}^{-1}$ and $\Omega=304 \mathrm{~K}$. With these constants $\alpha_{\mathrm{L}}$ equals $1.02,5.85$, and $8.62 \times 10^{-6} \mathrm{~K}^{-1}$ at the temperatures of, respectively, $100 \mathrm{~K}, 200 \mathrm{~K}$, and $300 \mathrm{~K}$.

Another set of volumetric thermal expansion coefficients is found in the paper by Suzuki, Anderson, \& Sumino (1983), already mentioned in the subsection on Young's modulus. Their data refer to the crystal of forsterite and cover the desired range of temperatures. A fit by Eq. (43) requires that $\lambda=9.14 \times 10^{-6} \mathrm{~K}^{-1}$ and $\Omega=251.4 \mathrm{~K}$. The fit gives $\alpha_{\mathrm{L}}$ equal to $1.53,5.54$, and $7.27 \times 10^{-6} \mathrm{~K}^{-1}$ at $100 \mathrm{~K}, 200 \mathrm{~K}$, and $300 \mathrm{~K}$, respectively. The fit to the measured values is perfect for the first two temperatures, but too low by 12 percent for the third.

Unfortunately, most information available on the thermal expansion coefficient refers to geology of the earth's crust, volcanology, and related scientific fields (e.g., Afonso, Ranalli, \& Fernàndez 2005). As a result, one seldom finds data pertaining to low temperatures and low pressures. In addition, the effect of porosity on thermal expansion is seldom addressed, and when it is, the results and implications depend on the type of material and physical conditions (e.g., Faivre, Bellet, \& Dolino 2000; Moretti et al. 2005; Hunter \& Brownell 2006; Ghabezloo \& Sulem 2009; Ghabezloo 2010), although generally there appears to be a tendency for the thermal expansion coefficient to increase with increasing porosity.

Poisson's ratio. Besides the elastic moduli, Yomogida $\&$ Matsui (1983) also measured Poisson's ratio $\nu$ for the already mentioned 20 meteorites. With the exception of a single anomalous sample (the same one that did not fit the dependence of Young's modulus on porosity), all 19 remaining had their values of $\nu$ between 0.12 and 0.29 . In this data set there is a slight tendency for $\nu$ to decrease with increasing porosity, but the scatter is too large for a functional fit; on the average, $\langle\nu\rangle=0.21 \pm 0.05$.

For olivine, Chung (1970) found that Poisson's ratio was increasing systematically with the $\mathrm{Fe} / \mathrm{Mg}$ ratio from 0.242 for forsterite to 0.308 for fayalite. Just as with the thermal expansion coefficient, the dependence of Poisson's ratio on porosity for terrestrial materials appears to vary from case to case. Gel derived silica, for example, has Poisson's ratio nearly constant between 0.15 and 0.19 at porosities smaller than 0.6 , but it then rapidly climbs to 0.26 at porosity 0.82 (Ashkin, Haber, \& Wachtman 1990; Phani \& Sanyal 2005). On the other hand, Poisson's ratio of sintered iron decreases from 0.30 at zero porosity to 0.20 at porosity 0.2 (Kováčik 2005).

The temperature dependence of Poisson's ratio for forsterite is shown by Suzuki, Anderson, \& Sumino's (1983) data. The rate amounts to $<0.001$ per $100 \mathrm{~K}$ and can be neglected. Compared to Young's modulus and the thermal expansion coefficient, Poisson's ratio has a relatively minor effect in computing thermal stress.

Adopting stress parameter $\chi$ for $\mathrm{C} / 2011$ W3. Whereas the computed relative variations in thermal stress in the interior of comet $\mathrm{C} / 2011$ W3 in Fig. 14 suggest an enormous growth near and after perihelion, it remains unclear whether the effect is sufficient to crumble and collapse the nucleus. Investigations of this kind can only be conducted in a framework of a particular comet model, with realistic estimates for the quantities that enter the stress parameter $\chi$. The extensive discussion of Young's modulus, the thermal expansion coefficient, and Poisson's ratio was intended to facilitate this effort.

To anchor our efforts in the framework of a numericalsimulation construct for comet formation, evolution, and morphological makeup, we focus on Lasue et al.'s (2009, 2011) work on aggregation and collisional interaction of cometesimals in the primordial solar nebula and the internal structure of cometary nuclei. Their developed model is based on a homogeneity exponent determining an aggregation regime, accounts for disruptive, sticking, compaction, and sintering processes, allows comparison with observational constraints and future in situ observations, and provides predictions for the cohesive strength and the radial porosity profile of the nucleus' interior. In our choice of parameters we concur with Lasue et al., who clearly prefer the layered structure of the nucleus, which is determined by the range of 0.4 to 0.6 for their homogeneity exponent and which offers the characteristic values of the porosity and cohesive strength that we use in this study.

Keeping in mind the complete disintegration of $\mathrm{C} / 2011$ W3 after perihelion, our primary interest is the temporal variation in thermal stress deep inside the nucleus, which we refer to as the central thermal stress. By this term we understand stress in the volume of the nucleus that does not include the surface layer, where the thermal stress profile is very different from that at greater depths. In Fig. 14 the boundary between the surface layer and the deep interior approximately coincides with the apparent minimum on the stress curve, associated with the transition of the tangential component $\sigma_{\perp}$ from compression to tension.

The extensive areas of the surface and the adjacent, relatively shallow subsurface layer of the nucleus that are presumed to be devoid of ices, must in the proximity of perihelion suffer from even higher levels of thermal stress than the rest of the nucleus (Fig. 14). They are primary candidates for thermal-stress damage, being probably riddled with crisscross cracks and the debris removed soon after perihelion. These areas may in fact provide for the comet's earliest postperihelion activity. We will not deal with them in this paper.

In Fig. 14, a rather interesting property of the thermal stress distribution in deeper layers, say, from a depth of $\sim 30$ meters on, is that the magnitude of the total effect,

$$
\sigma=\sqrt{\sigma_{\|}^{2}+\sigma_{\perp}^{2}},
$$

is almost independent of depth, especially at perihelion and afterwards. Because $\sigma$ fairly rapidly converges to $\sigma_{\|} \sqrt{2}=\sigma_{\perp} \sqrt{2}$ at the nucleus' center, it is both convenient and appropriate to deal with this limit as a measure of thermal stress in our consideration of its temporal variations in absolute units - hence the reason for the term central.

The discussion of the quantities that make up the stress parameter $\chi$ suggests that in an effort to find their most appropriate values one will encounter considerable difficulties and uncertainties. The two most severe problems are the strong dependence of Young's modulus on poros- 
ity and the thermal expansion coefficient's variation with temperature. In the former case, there is the need to extrapolate the data way outside the covered range of porosity. In the latter instance, it is troubling to find a parameter, which the thermal stress theory handles as a constant, to be so strongly temperature dependent. Only the selection of a value for Poisson's ratio appears to be somewhat less controversial, thanks to this quantity's relative invariability. Yet, for all three quantities it is necessary to resort to other classes of objects and/or substances to approximate cometary material. Also, we have no choice but to rely on a comet model to estimate the porosity in the interior. With these caveats in mind we proceed to the next step.

We note that Lasue et al.'s (2011) aggregate model predicts a porosity close to 0.65 at large depths and near the center of a cometary aggregate regardless of whether the size distribution function of the cometesimals is Gaussian or a power law. Equation (41) then yields $E_{\mathrm{Y}}=8.6$ $\mathrm{MPa}$ from the high $E_{\mathrm{Y}}$ group of meteorites and $E_{\mathrm{Y}}=3.1$ $\mathrm{MPa}$ from the low $E_{\mathrm{Y}}$ group. For the coefficient of linear thermal expansion $\alpha_{\mathrm{L}}$ we have to adopt a mean value from a range of less than 1 to at least 6 or $7 \times 10^{-6} \mathrm{~K}^{-1}$, based on Suzuki, Anderson, \& Sumino's (1983) data for a single crystal of forsterite. Because of the possibility that an explosion from a reservoir of amorphous water ice at depths in excess of 40 meters was a trigger of, or a contributor to triggering, the terminal outburst (Fig. 12 ), we have chosen $\alpha_{\mathrm{L}}=3 \times 10^{-6} \mathrm{~K}^{-1}$, which is near the mean and is also attained at $130 \mathrm{~K}$, the temperature of activation of the ice's exothermic process of crystallization (e.g., Schmitt et al. 1989). Because of the dearth of relevant information, we have not included any porosity correction. For Poisson's ratio we have adopted $\nu=0.1$, which is based primarily on the sample of meteorites and allows for an assumed modest decrease with porosity. Its value has the role of a minor correction to the stress parameter, which comes out to be $\chi=29 \mathrm{~Pa}$ and $10 \mathrm{~Pa}$ with the two values of $E_{Y}$.

To address the issue of whether the magnitude of thermal stress determined in this way for $\mathrm{C} / 2011$ W3 could lead to the crumbling and collapse of this comet's nucleus, we have compared our results with the expected cohesive strength of a cometary aggregate provided by Lasue et al.'s model. Specifically, we have taken approximate upper and lower limits near the homogeneity exponent of 0.5 , as presented in Fig. $7 \mathrm{~b}$ of Lasue et al. (2009), because they alluded to this point only in general terms in their second paper (Lasue et al. 2011); the understanding the reader gets is that the introduction of a size distribution function of cometesimals had no significant effect on the cohesive strength.

The resulting thermal stress curves, referring to the two adopted values of Young's modulus, are plotted against time from perihelion in Fig. 15. In broad terms, we conclude that thermal stress developing in the interior of the nucleus is: (1) insufficient to disrupt the object before perihelion; (2) comparable to the cohesive strength in the immediate proximity (within hours) of perihelion, thus weakening the nucleus' structure; and (3) greater than the strength of the comet after perihelion to the extent that it can no longer hold together.

Because of the uncertainties involved, one needs to ex- ercise a caution and emphasize that this conclusion is model and parameter dependent. Nevertheless, in the case of the thermal expansion coefficient, its chosen constant value has a tendency to suppress the degree of asymmetry of thermal stress relative to perihelion, that is, it overestimates thermal stress before perihelion and underestimates it after perihelion. As a matter of fact, this relation can be quantified: from Eq. (43) it follows that a change $\Delta T$ in the temperature leads to the following change $\Delta \alpha_{\mathrm{L}}$ in the coefficient of linear thermal expansion:

$$
\frac{\Delta \alpha_{\mathrm{L}}}{\alpha_{\mathrm{L}}}=2\left[\frac{\Omega}{T} \operatorname{coth}\left(\frac{\Omega}{T}\right)-1\right] \frac{\Delta T}{T} .
$$

This formula indicates that, for example, a modest temperature increase of $5 \mathrm{~K}$ entails an increase in the relative rate of the $\alpha_{\mathrm{L}}$ coefficient - and therefore in thermal stress - of 2.4 percent at $200 \mathrm{~K}, 5.3$ percent at $150 \mathrm{~K}$, 15.5 percent at $100 \mathrm{~K}$, and an enormous 37 percent at $70 \mathrm{~K}$. This effect comes on top of the thermal stress increase that we have discussed below Eq. (35), and enhances the role of thermal stress as the driving force behind the events of cascading fragmentation at large heliocentric distances, as mentioned in point (5) between Eqs. (40) and (41); it also aids the role of thermal stress in unlocking potential reservoirs of highly volatile ices in the nucleus' interior.

In summary, our suspicion that the delayed response of the nucleus of $\mathrm{C} / 2011$ W3 to the extremely high temperatures in the proximity of perihelion was due to heating its interior and to the resulting effect of thermal stress finds support in this comprehensive analysis, which is limited only by incomplete information on cometary material - the problem that no hypothesis can avoid. If our scenario is valid at least with an order-of-magnitude accuracy, it strengthens the notion that $\mathrm{C} / 2011 \mathrm{~W} 3$ was able to withstand thermal stresses on the order of several $\mathrm{kPa}$ and therefore possessed far more cohesion than necessary to avoid collapse and disintegration right at perihelion, in the inner solar corona.

\section{STANDING OF C/2011 W3 IN THE KREUTZ SYSTEM} AND ITS POSSIBLE PAST EVOLUTIONARY PATH

The place of the now defunct comet C/2011 W3 in the hierarchy of the Kreutz system of sungrazers cannot be pinpointed with certainty. However, within a given model of the system, the comet's evolutionary path can be traced in some detail. The exercise involves modeling and interpretation of long-term changes in (a) the orbital period and (b) the angular elements and perihelion distance. As amply explained in Sec. 1, the first issue is addressed in terms of the tidal-assisted fragmentation of the early parent objects of $\mathrm{C} / 2011$ W3 in the close proximity of perihelion, while the second issue involves a sequence of fragmentation episodes of its more recent precursors far from the Sun.

As an example, we elaborate on the evolutionary path of C/2011 W3 in the context of the hierarchical scenario $\mathrm{B}$ of the Kreutz system, introduced in our recent paper (Sekanina \& Chodas 2007). This scenario is based on the postulated identity of the spectacular sungrazer X/1106 C1 with a comet of A.D. 467. The true orbital period of this progenitor (or superfragment, in the terminology used in Sekanina \& Chodas 2004) was obviously 


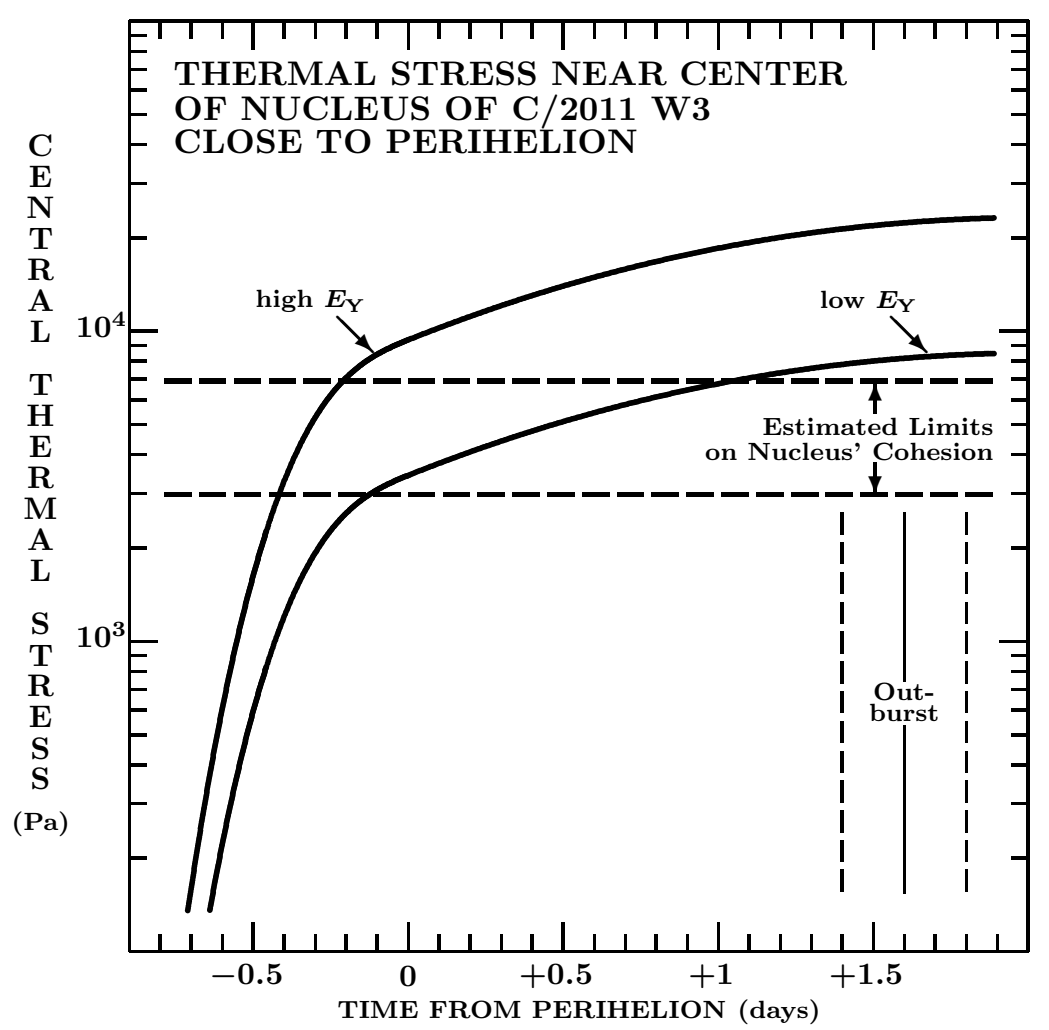

Figure 15. Computed thermal stress near the center of the nucleus of C/2011 W3. The solid curves show the stress growth through perihelion for the two adopted values of Young's modulus, the horizontal dashed lines mark the estimated limits on the cohesive strength of the nucleus (from Lasue et al. 2009, 2011). The stress curves represent a conservative estimate; because the thermal expansion coefficient increases with the temperature, the actual postperihelion growth of thermal stress in the comet's interior is even steeper. The time of the terminal outburst of $\mathrm{C} / 2011 \mathrm{~W} 3,1.6 \pm 0.2$ days after perihelion, is shown by the solid vertical line, its uncertainty by the parallel broken lines.

639 years. The integration of the orbit of $\mathrm{C} / 2011 \mathrm{~W} 3$ back in time indicates (Table 4 ) that the previous return to perihelion occurred in January 1329 (with a formal uncertainty of \pm 2 years, which for this exercise is not critical). It should be emphasized that the 1329 orbital set is valid only in the unlikely absence of any fragmentation events involving the comet's precursors during the entire revolution about the Sun. Only in this hypothetical case does the true orbital period come out to be almost exactly 683 years. In order for a fragment - an early parent of $\mathrm{C} / 2011 \mathrm{~W} 3$ - of comet 467 to arrive at perihelion in 1329 , its true orbital period must have been 862 years, or 223 years longer than that of the principal fragment, comet X/1106 C1. This increase in the orbital period requires that the parent fragment of $\mathrm{C} / 2011 \mathrm{~W} 3$ split off from the main body of comet 467 near perihelion (say, at a heliocentric distance of $0.01 \mathrm{AU}$ ) with a separation velocity of $\sim 2.2 \mathrm{~m} \mathrm{~s}^{-1}$ essentially in the direction of the orbital motion (Table 8 of Sekanina 2002a). When this parent fragment arrived at perihelion in 1329 , its orbital period was (with some uncertainty due to the indirect planetary perturbations) still 862 years. Since the true orbital period of $\mathrm{C} / 2011 \mathrm{~W} 3$ is 683 years, the necessary decrease of 179 years in the orbital period requires that a new fragment broke off from the parent fragment in 1329, again in the immediate proximity of perihelion, with a separation velocity of $\sim 3.6 \mathrm{~m} \mathrm{~s}^{-1}$ essentially in the direction opposite the direction of orbital motion. Separation rates of fragments corresponding to relative velocities of up to 5 or $6 \mathrm{~m} \mathrm{~s}^{-1}$ are known to be necessary for explaining the tidal-assisted splitting of sungrazing comets (Sekanina \& Chodas 2007), so the required velocities of less than $4 \mathrm{~m} \mathrm{~s}^{-1}$ provide a plausible evolutionary model for comet C/2011 W3. Similar numbers would result if the adopted scenario $\mathrm{B}$ should be replaced with another one.

Because of potential differences in the angular elements and perihelion distance between the orbits of $\mathrm{C} / 2011 \mathrm{~W} 3$ and the new fragment referred to above, it is highly unlikely that the two objects are identical. Much more probable is that the new fragment was a precursor to C/2011 W3, which between 1329 and 2011 underwent additional, nontidal fragmentation at large heliocentric distances, and that during these episodes one of the fragments acquired the necessary orbital orientation and perihelion distance to become $\mathrm{C} / 2011 \mathrm{~W} 3$. If the first precursor's angular elements in the 14th century were in the range of most SOHO sungrazing comets, that is, close to those of $\mathrm{C} / 1963 \mathrm{R} 1$, the differences relative to $\mathrm{C} / 2011$ W3 may have been substantial, about $35^{\circ}$ to $40^{\circ}$ in the argument of perihelion and the longitude of the ascending node, some $10^{\circ}$ in the inclination, and up to 10 percent of the Sun's radius in the perihelion distance. To bridge the gaps of this magnitude requires several nontidal fragmentation events in the general proximity of aphelion (at heliocentric distances of, typically, 100 AU or so) at an assumed average separation velocity of $2-3 \mathrm{~m}$ $\mathrm{s}^{-1}$, a requirement by no means excessive. A simulation 


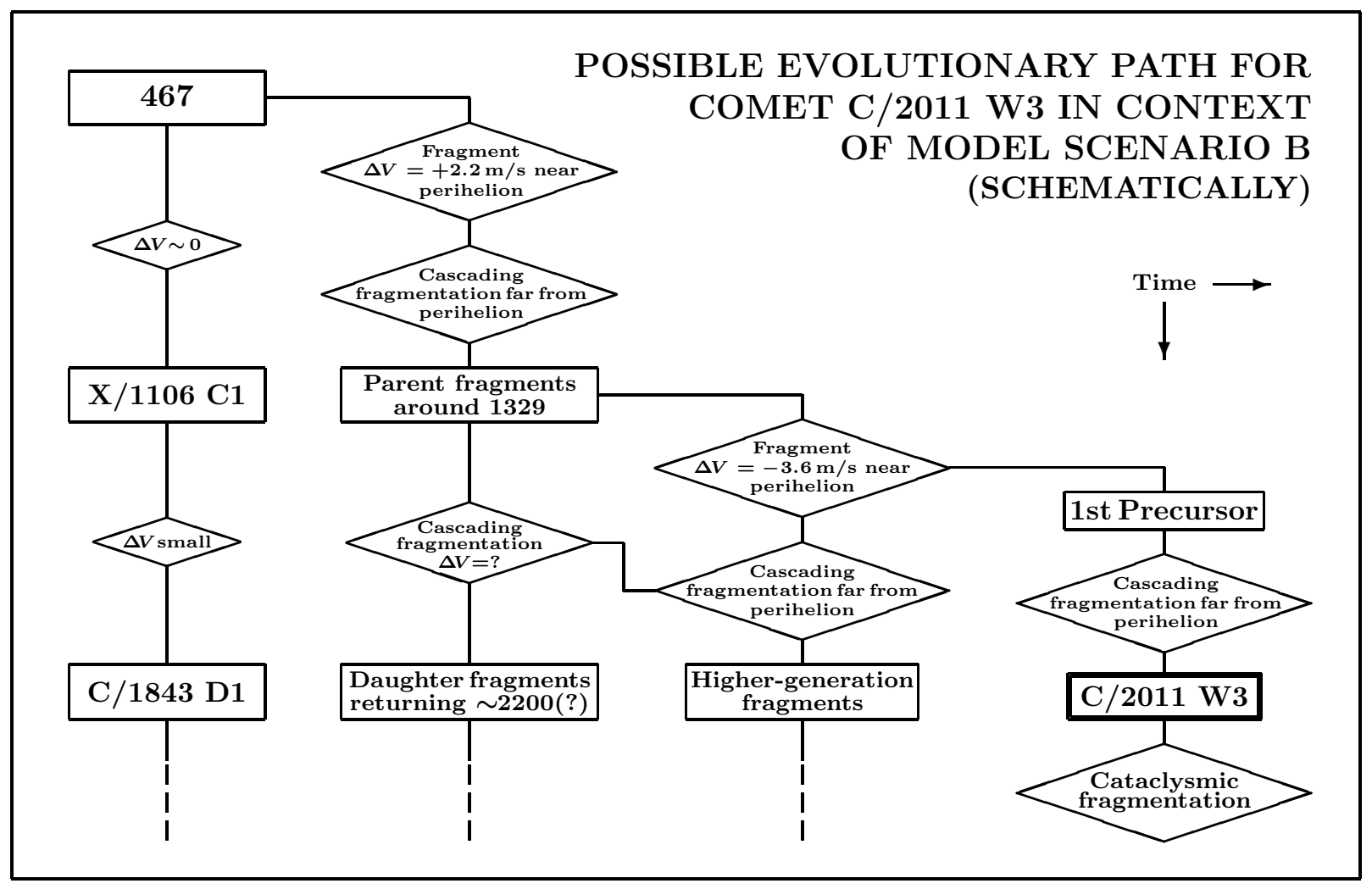

Figure 16. Possible evolutionary path for comet C/2011 W3 in a broader context of the model scenario B (Sekanina \& Chodas 2007), which assumes that comet $\mathrm{C} / 1843 \mathrm{D} 1$, rather than $\mathrm{C} / 1882 \mathrm{R} 1$, is the most massive fragment of X/1106 C1. The parallel fragmentation branch involving $\mathrm{C} / 1882 \mathrm{R} 1$ is not shown in this schematic representation. The diamond-shaped boxes describe events of the tidal-assisted and cascading nontidal fragmentation processes, the rectangular boxes are their products. The separation velocities involved are given as $\Delta V$; the sign refers to the direction along the orbital-velocity vector: positive means ahead, negative behind. The fragmentation hierarchy is presented schematically, with only some paths among the great multitude of possible chain events being depicted.

model for a sequence of nontidal fragmentation events scaled for the Kreutz system from the case of D/1993 F2 (Shoemaker-Levy 9) shows that the number of these episodes can easily exceed a dozen during one revolution about the Sun (Sekanina 2002a, 2002b). Their high frequency is also obvious from the arrival rate of the $\mathrm{SOHO}$ minicomets, the high-generation products of the cascading fragmentation process, the age of each of them being manifestly shorter than one revolution about the Sun (e.g., Sekanina \& Chodas 2004).

In the broader context of the fragmentation hierarchy of the branch of the Kreutz system, which in our Scenario $\mathrm{B}$ also contains the sungrazer C/1843 D1, the described evolutionary path of $\mathrm{C} / 2011$ W3 is depicted in Fig. 16. In this more general scheme, the parent fragment may have reached perihelion years or even decades before or after 1329, because fragmentation at large heliocentric distances entails modest changes in the orbital period of up to a few years per event (Table 8 of Sekanina 2002a).

Regardless of the details of the evolution of C/2011 W3, its origin appears to be linked to the expected new, 21st century cluster of bright sungrazers. What remains unclear is its subgroup membership. While already Kreutz (1901) was thinking in terms of two subgroups when investigating historical sungrazers, this division for the bright members of the Kreutz system became a hit after Marsden's (1967) publication of his classical paper, which listed C/1843 D1, C/1880 C1, and C/1963 $\mathrm{R} 1$ as the definite members of subgroup I, while $\mathrm{C} / 1882$ $\mathrm{R} 1, \mathrm{C} / 1945 \mathrm{X} 1$, and $\mathrm{C} / 1965 \mathrm{~S} 1$ belonged to subgroup II. In addition, Marsden tabulated four and two additional comets, respectively, as possible members of the two categories. A dent in this classification scheme was made by C/1970 K1, whose orbit did not fit either subgroup. Because its orbit was closer to subgroup II, Marsden (1989) classified it subsequently as subgroup IIa. Now comes $\mathrm{C} / 2011 \mathrm{~W} 3$, whose perihelion distance fits subgroup I, but the angular elements are incompatible with any of the subgroups I, II, or IIa. This is worrisome, because Marsden (1967) regarded the longitude of the nodal line, one of the orbital angles, as the prime classifier for the subgroups. If one considers, by extension, C/2011 W3 to be a representative of a new subgroup III, one ends up with a total of eight bright sungrazers distributed into four categories, or two members per subgroup on the average, not to mention that the orbit of $\mathrm{C} / 1945 \mathrm{X} 1$, one of the eight, is not really all that well fixed (see Marsden 1989 and our comment about Cunningham's computations in Sec. 7).

An overwhelming majority of SOHO, SMM, and SOLWIND sungrazers belongs to subgroup I. This was shown to be the case by Marsden (1989) for the SMM and SOLWIND comets and is illustrated for 1565 SOHO sungrazers in a plot of the longitude of the ascending node 


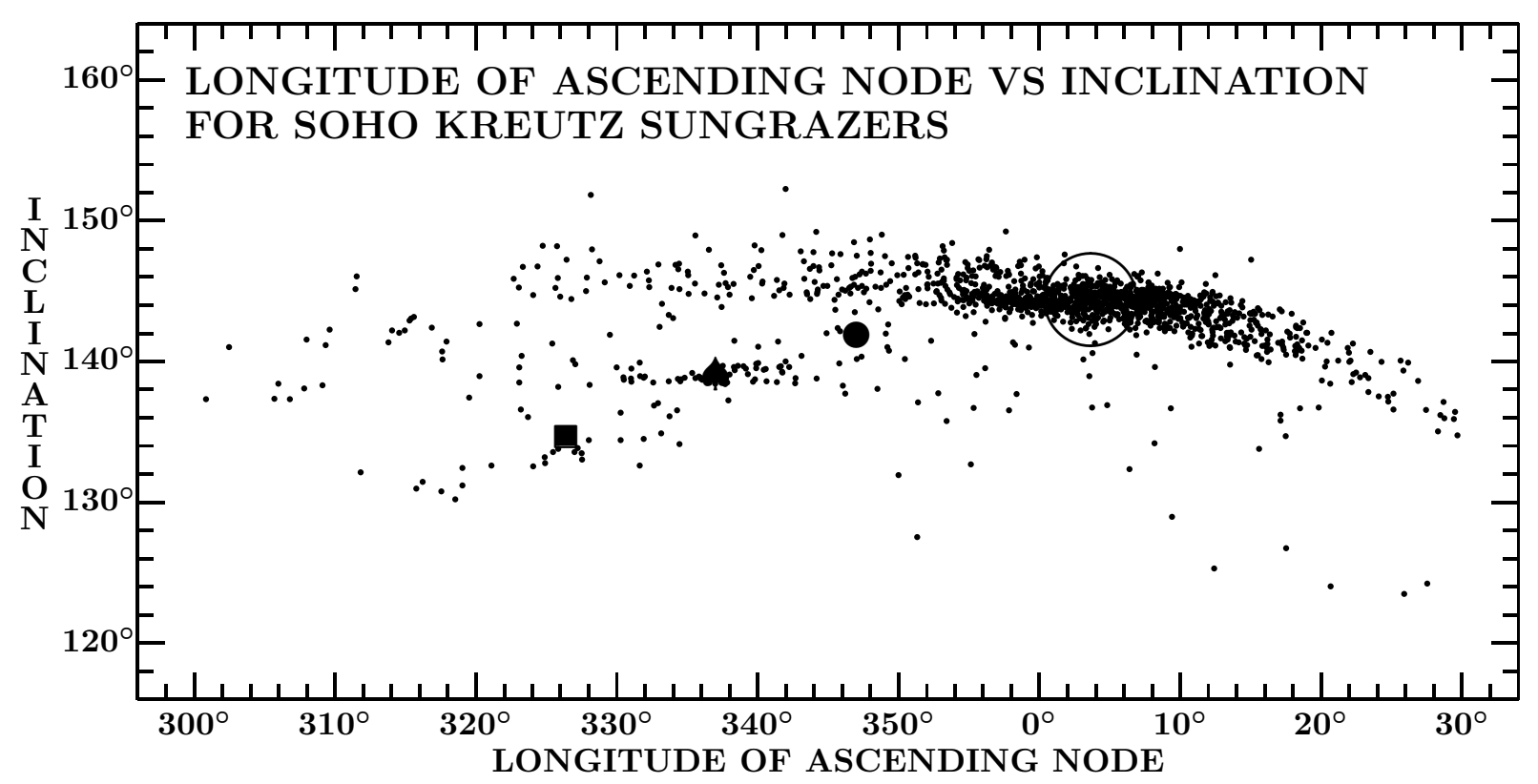

Figure 17. Comets C/2011 W3, C/1970 K1, C/1965 S1, and C/1843 D1 in a plot of the longitude of the ascending node against the orbit inclination for 1565 SOHO Kreutz sungrazers from the period January 1996 to June 2010. C/2011 W3 is identified by the square, C/1970 K1 by the spadesuit mark, C/1965 S1 by the filled circle, while the location of C/1843 D1 coincides with the dense concentration of the SOHO sungrazers and is in the center of the open oversized circle. Comet C/2011 W3 appears to be orbitally related to a cluster of up to nearly $30 \mathrm{SOHO}$ sungrazers with the ascending nodes between $310^{\circ}$ and $335^{\circ}$ and with the inclinations between $130^{\circ}$ and $137^{\circ}$.

against the orbit inclination in Fig. 17. However, nearly 100 SOHO sungrazers, or about 6 percent of the total, make up a second branch, which in the range of ascending node longitudes from $\sim 320^{\circ}$ to at least $\sim 345^{\circ}$ lies about $6^{\circ}$ below an extended arm of the subgroup I set. And some $4^{\circ}$ to $5^{\circ}$ below this second branch there is yet another cluster of nearly $30 \mathrm{SOHO}$ sungrazers with their nodal longitudes between $310^{\circ}$ and $335^{\circ}$.

For the sake of comparison, we have also included four bright sungrazers in Fig. 17. Comet C/1843 D1, of subgroup $\mathrm{I}$, is right in the dense core of the $\mathrm{SOHO}$ sungrazers, while C/1965 S1 (of subgroup II), C/1970 K1 (of subgroup IIa), and $\mathrm{C} / 2011 \mathrm{~W} 3$ are all located off the main branch. A surprising finding is that the second, much less populated but still well defined branch of the SOHO sungrazers belongs to subgroup IIa (not II, as has generally been assumed). There is practically no concentration at all of the SOHO objects near the location of $\mathrm{C} / 1965 \mathrm{~S} 1$. On the other hand, the agreement between the positions of $\mathrm{C} / 2011 \mathrm{~W} 3$ and a third, very sparsely populated branch is rather obvious. Thus, when it comes to the SOHO sungrazers, the orientation of the orbit of $\mathrm{C} / 2011$ W3 in space is certainly not unique. And while the three branches essentially merge into a common relation in the plot of the longitude of the ascending node against the argument of perihelion in Fig. 18, C/2011 W3 is again surrounded by a number of SOHO sungrazers.

On the whole, the classification of the bright sungrazers into the subgroups is somewhat questionable. A suggestion for its eventual abandonment is supported by our finding (Sekanina \& Chodas 2007) that even following a single fragmentation event at large heliocentric distance, two fragments of the same parent can end up in orbits formally belonging to different subgroups. In fact, the progenitor's splitting far from the Sun into two superfragments with widely different orbits is a prerequisite behind the idea of introducing the concept of subgroups in the first place (Sekanina \& Chodas 2004).

The point of contention with $\mathrm{C} / 2011 \mathrm{~W} 3$ is thus not whether it could derive from a precursor belonging to another subgroup, but, rather, that no plausible candidate for a sungrazing comet was ever recorded in the first half of the 14th century. Hasegawa \& Nakano (2001) considered only one possible sungrazer, X/1381 V1, during the entire 14th century. All three comets between 1282 and 1368 in England's (2002) list have a relatively low sungrazer ranking, even though the author does not entirely exclude the possibility for each of them, including one seen in Europe in October 1314, to be a member of the Kreutz system.

On the other hand, only if the cumulative effect in the orbital period from a number of fragmentation episodes at large heliocentric distances could, in an extreme and quite unlikely run of events, amount to as much as $\sim 50$ years, could $\mathrm{X} / 1381 \mathrm{~V} 1$ have been a parent to $\mathrm{C} / 2011$ W3 in the sense that one of the products of its tidalassisted breakup in 1381 would have become the first precursor to $\mathrm{C} / 2011 \mathrm{~W} 3$.

Of course, a large number of comets recorded in the late 13 th century and during the 14 th century were cataloged by Ho (1962), by Hasegawa (1980), and by Kronk (1999). However, most of them fail to fit a Kreutz sungrazer because of the time of the year, the location in the sky, the apparent motion, or the appearance, while for the rest of them the reported information is too vague for the identification. The future modeling of the Kreutz-system evolution should proceed with these circumstances taken into account. 


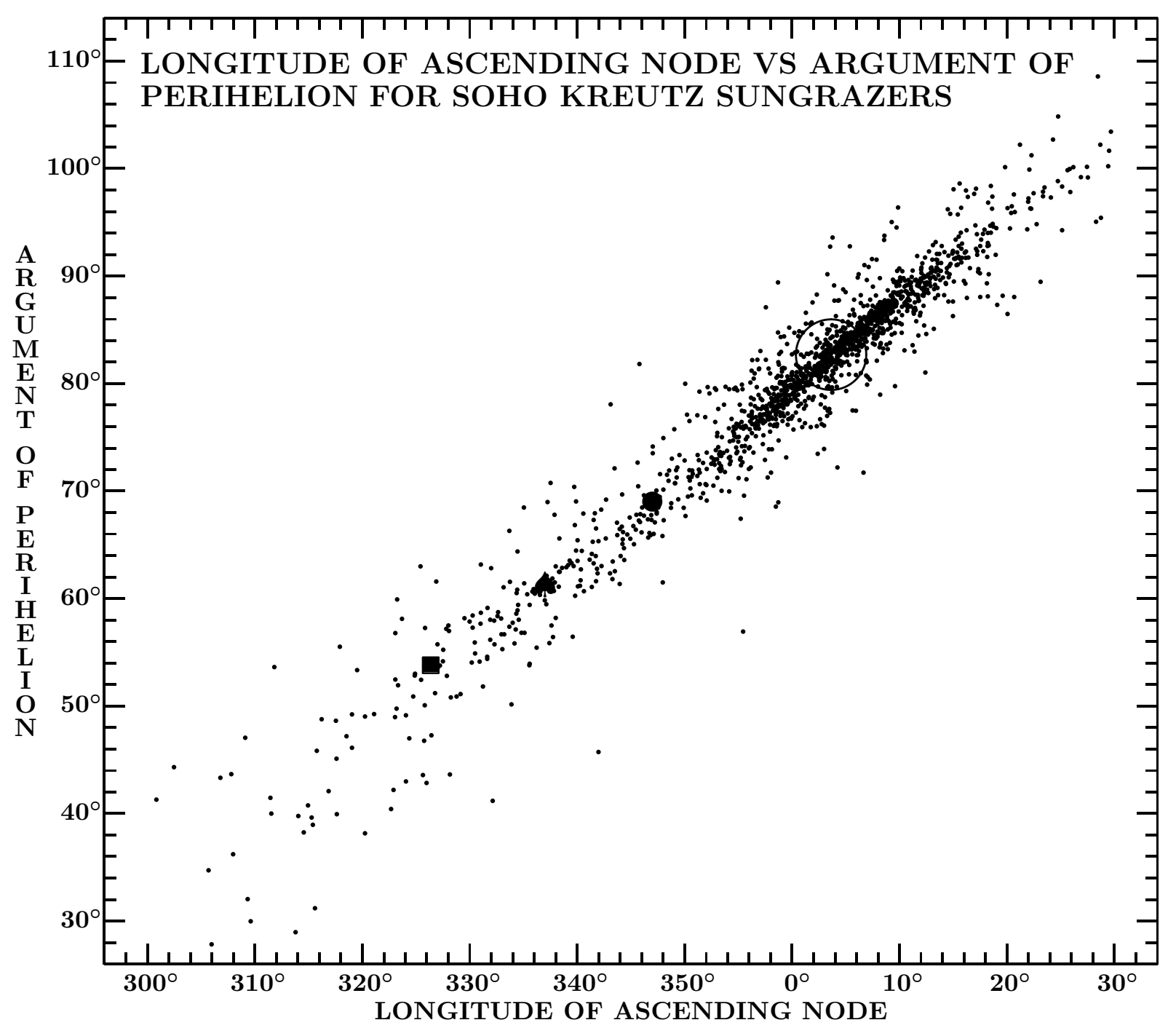

Figure 18. Comets C/2011 W3, C/1970 K1, C/1965 S1, and C/1843 D1 in a plot of the longitude of the ascending node against the argument of perihelion for 1565 SOHO Kreutz sungrazers from the period January 1996 to June 2010. The four bright comets are identified the same way as in Fig. 17. Although the three branches now essentially merge into one relation, the position of C/2011 W3 in the plot appears to be again closely matched by a modest number of SOHO sungrazers.

\section{CONCLUSIONS}

The issues and results presented in this investigation are summarized into the following conclusions:

(1) Having survived the perihelion passage, comet C/2011 W3 was observed to undergo major morphological changes, culminating in the permanent loss of the nuclear condensation between 2011 December 19.4 and 20.3 UT, some 4 days after perihelion.

(2) This transformation was accompanied by a parallel development of a narrow, rectilinear dust streamer, extending essentially along the tail's axis in the images of December 19 and rapidly evolving, in less than 24 hours, into a ribbon-like spine tail.

(3) From the temporal variations in the projected orientation of its axis between December 19 and January 18, we find that this spine tail was a synchronic feature, whose brightest part consisted of submillimetersized dust grains released at velocities not exceeding 30 $\mathrm{m} \mathrm{s}^{-1}$ and whose origin was an outburst or a terminal (cataclysmic) fragmentation event (possibly even a rapid sequence of such very brief episodes), which peaked on December $17.6 \pm 0.2 \mathrm{UT}$.

(4) Accordingly, the comet's postperihelion activity, which lasted less than 48 hours and resulted in releasing up to an estimated $10^{12}$ grams of dust (equivalent to a sphere 150-200 meters across), was the sole source of the spectacular dust tail, which was observed for 3 months, until mid-March.

(5) The delayed response of $\mathrm{C} / 2011 \mathrm{~W} 3$ to the extremely adverse environment in the close proximity of the Sun, which the nucleus' disintegration must have signified, is of utmost importance. This overdue reaction speaks volumes about the cohesion of the nucleus and provides a clue to the probable nature of the breakup mechanism - as a product of a gradual heat pulse penetration to great depths of the nucleus and an entailing steady buildup of thermal stress throughout the interior.

(6) Modeling of the heat-transfer process and the thermal stress distribution inside the nucleus, whose dimen- 
sions diminish with time because of continuing erosion at the surface, shows that the rate of propagation of the heat pulse around perihelion was extremely rapid and highly asymmetric in that at greater depths beneath the surface both temperature and thermal stress were much higher at the same heliocentric distance after than before perihelion and continued to grow far from the Sun, on the way to aphelion.

(7) Based on the elastic constants for meteoritic, lunar, and some terrestrial analogs, the magnitude of thermal stress throughout much of the nucleus is estimated at more than $10 \mathrm{kPa}$ shortly after perihelion, overcoming the object's expected cohesive strength (on the order of several $\mathrm{kPa}$ according to Lasue et al.'s aggregate model), riddling the entire interior with a dense network of clefts and cracks, thus causing eventually the nucleus' collapse into a cloud of debris.

(8) The same mechanism may be responsible for the ubiquitous process of cascading fragmentation that sungrazers are subjected to at all heliocentric distances, including very far from the Sun.

(9) On the other hand, survival in the solar corona and the delayed response to the adverse conditions in its environment is inconsistent with the model of a strengthless or poorly cemented rubble-pile nucleus made up essentially of unconsolidated debris.

(10) If there were isolated reservoirs of volatile ices deep in the interior of the nucleus, major cracks caused by excess thermal stress would have opened them to the propagating heat wave, with the ensuing explosion contributing to the destruction of the nucleus; our model predicts that 1.6 days after perihelion, pockets of amorphous water ice more than 40 meters beneath the surface would have been exposed to a temperature of $\sim 130 \mathrm{~K}$ and thereby an exothermic reaction would be triggered, associated with amorphous ice's crystallization; only data on temporal variations in the comet's production of water vapor and/or other gas species could settle this issue.

(11) The disappearance of the nuclear condensation deprived observers of obtaining postperihelion astrometry needed for an accurate orbit computation by traditional techniques, so that the orbital period of $\mathrm{C} / 2011$ W3 remained essentially indeterminate even at the time of termination of observations.

(12) We argue that the missing nucleus must have been located on the synchrone defined by the axis of the spine tail whose orientation and sunward tip have been measured; the missing nucleus also must have been situated on the line of forced orbital-period variation, computed from the orbital solutions based on high-quality preperihelion astrometry from the ground.

(13) We succeed in deriving the astrometric positions of this missing nucleus as the coordinates of the points of intersection of the spine tail's synchrone and the line of orbital-period variation; a high-quality orbital solution is obtained by linking the preperihelion observations with these derived positions of the missing nucleus.

(14) The resulting orbit gives $698 \pm 2$ years for the osculating orbital period and confirms that $\mathrm{C} / 2011 \mathrm{~W} 3$ is the first major member of the expected new, 21st century cluster of bright Kreutz-system sungrazers, whose existence was predicted by these authors in 2007 .

(15) From the spine-tail evolution we determine that the measured sunward tip receded antisunward from the computed position of the missing nucleus, the distance increasing from $1^{\prime}$ on December 23 to more than $10^{\prime}$ in mid-January and that this terminus was populated by dust particles 1-2 $\mathrm{mm}$ in diameter.

(16) The bizarre appearance of the comet's dust tail in images taken with the coronagraphs on board the SOHO and STEREO spacecraft only hours after perihelion is modeled, using the standard dynamical method, in terms of the ratio $\beta$ of the acceleration by solar radiation pressure to that by the solar gravitational attraction; the tail brightness was affected by forward scattering of sunlight by dust to a considerable degree.

(17) Modeling of the SOHO's C3 preperihelion images of the dust tail shows a population of predominantly microscopic particles with a broad range of sizes and composition, described both by $\beta>1$ (strongly absorbing, such as carbon-rich, grains moving in convex hyperbolic orbits) and by $\beta<1$ (essentially dielectric, perhaps mostly silicate, grains in concave hyperbolic orbits); the ones that were still imaged after perihelion had $\beta \simeq 0.6$, probably submicron-sized silicate particles.

(18) By contrast, nearly all microscopic dust released before perihelion into convex hyperbolic orbits moved almost immediately away from the Sun and was not seen in the postperihelion images of the comet.

(19) The disconnection of the comet's head from the dust tail released before perihelion, strikingly depicted in the postperihelion images taken with the SOHO's $\mathrm{C} 2$ coronagraph and with the coronagraphs on board STEREO-A and STEREO-B, is interpreted as a result of vigorous sublimation of submicron-sized dust at heliocentric distances smaller than about $1.8 R_{\odot}$, primarily particles with $\beta \leq 0.6$ released between 0.1 day before and 0.1 day after perihelion.

(20) From the integrated effect of dust particle sublimation, we establish the relationship between the sublimation-pressure constant $\Lambda$ and the heat of sublimation $L$ and find that it is consistent with the $L(\Lambda)$ relation for $\mathrm{Mg}$-rich olivine and that therefore it is likely that the dust sublimating very close to the Sun is dominated by olivine-based silicates, a conclusion that is also supported dynamically by the magnitude of the acceleration parameter $\beta$.

(21) The place of $\mathrm{C} / 2011 \mathrm{~W} 3$ in the hierarchy of the Kreutz system of sungrazing comets is a matter of some speculation, but one possible evolutionary path is charted, based on our orbit integration back to the comet's previous return to perihelion, which nominally occurred in 1329.

(22) In the context of Scenario B of the Kreutz system's hierarchy (from our 2007 paper) we follow the tidalassisted breakup of the progenitor (or superfragment) the comet of 467 - and the expected chain of nontidal fragmentation events into a number of parent fragments (including X/1106 C1); a subsequent tidal-assisted splitting of one such parent fragment in the 14th century into another generation of fragments, including the first precursor of C/2011 W3; and, finally, another bout of nontidal cascading fragmentation with the birth of comet $\mathrm{C} / 2011 \mathrm{~W} 3$ as a discrete object of its own.

(23) In this scenario, C/2011 W3 is indirectly related to $\mathrm{X} / 1106 \mathrm{C} 1$ and $\mathrm{C} / 1843 \mathrm{D} 1$, and in the coming years and decades it may be followed by more equally bright or brighter sungrazing comets in similar orbits. 
(24) Comet C/2011 W3 presents a complication for the classification of the bright sungrazers into subgroups: its perihelion distance is typical for subgroup I, but its angular elements do not fit any of the subgroups I, II, or IIa; thus, among the bright members of the Kreutz system, the orbit of $\mathrm{C} / 2011 \mathrm{~W} 3$ is unique, although we are not ready to call it a representative of a new subgroup.

(25) In a plot of the longitude of the ascending node against the inclination, C/2011 W3 is associated with the least populated of the three branches into which the SOHO sungrazers discriminate; from this standpoint, its orbit is not unique.

(26) Because the orbits of the sungrazers (including the bright objects) can be transformed by the cascading fragmentation process from one subgroup into another subgroup during one revolution about the Sun, the classification of the bright sungrazers into subgroups may no longer be appropriate.

(27) Future modeling of the hierarchy and evolution of the Kreutz system of sungrazers should account for the existence of $\mathrm{C} / 2011 \mathrm{~W} 3$ and its apparent relationship with the minor branch of SOHO sungrazers; at a minimum, orbital computations should verify the degree of plausibility of a fragmentation path of the type displayed in Fig. 16.

We thank S. R. Chesley for getting us acquainted with the correspondence he received from $R$. $H$. McNaught regarding the imaging with the Uppsala Schmidt Telescope, Siding Spring Survey, and in part via J. Giorgini, from M. Kusiak regarding his and R. Kracht's determination of the astrometric positions of the comet from the data collected on board the SOHO and STEREO spacecraft. We gratefully acknowledge Kracht's comments on his work concerning the SOHO and STEREO-B astrometry. We further thank J. Cerný and McNaught for their permission to reproduce the comet's postperihelion images taken, respectively, at Malargue and Siding Spring. D. W. E. Green kindly made available to us two older publications. This research was carried out at the Jet Propulsion Laboratory, California Institute of Technology, under contract with the National Aeronautics and Space Administration.

\section{REFERENCES}

Afonso, J. C., Ranalli, G., \& Fernàndez, M. 2005, Phys. Earth Plan. Inter., 149, 279

Aizawa, Y., Ito, K., \& Tatsumi, Y. 2001, Tectonophys., 339, 473

Ashkin, D., Haber, R. A., \& Wachtman, J. B. 1990, J. Amer. Ceram. Soc., 73, 3376

Asphaug, E., \& Benz, W. 1994, Nature, 370, 120

Asphaug, E., \& Benz, W. 1996, Icarus, 121, 225

Austin, J. B. 1952, J. Amer. Ceram. Soc., 35,xi 243

Barnes, L. 2012, http://oortcloud.org/index.php?topic=17617.0

Barthelmy, D. 2010, http://webmineral.com/data/Olivine.shtml

Biesecker, D. A., Lamy, P., St. Cyr, O. C., Llebaria, A., \& Howard, R. A. 2002, Icarus, 157, 323

Britt, D. T., \& Consolmagno, G. J. 2003, Meteorit. Plan. Sci., 38, 1161

Brownlee, D. E., Horz, F., Newburn, R. L., Zolensky, M., Duxbury, T. C., Sanford, S., Sekanina, Z., Tsou, P., Hanner, M.S., Clark, B. C., Green, S. F., \& Kissel, J. 2004, Science, 304, 1764

Brueckner, G. E., Howard, R. A., Koomen, M. J., Korendyke, C. M., Michels, D. J., Moses, J. D., Socker, D. G., Dere, K. P., Lamy, P. L., Llebaria, A., Bout, M. V., Schwenn, R., Simnett, G. M., Bedford, D. K., \& Eyles, C. J. 1995, Sol. Phys., 162, 357
Černý, J., ed. 2011, http://www.kommet.cz/page.php?al=prvni_ snimky_komety_lovejoy_ze_zeme

Chesley, S. R. 2012, personal communication

Chung, D. H. 1970, JGR, 75, 7353

Combi, M. R., DiSanti, M. A., \& Fink, U. 1997, Icarus, 130, 336

Consolmagno, G. J., Britt, D. T., \& Opeil, C. P. 2010, Eur. Plan. Sci. Congr. Abstr., 5, 215

Cremonese, G., Boehnhardt, H., Crovisier, J., Rauer, H., Fitzsimmons, A., Fulle, M., Licandro, J., Pollacco, D., Tozzi, G. P., \& West, R. M. 1997, ApJ, 490, L199

Cunningham, L. E. 1946a, IAUC 1025

Cunningham, L. E. 1946b, HAC 733

Davidsson, B. J. R., Gutiérrez, P. J., \& Rickman, H. 2009, Icarus, 201,335

England, K. J. 2002, JBAA, 112, 13

Faivre, C., Bellet, D., \& Dolino, G. 2000, J. Appl. Phys., 87, 2131

Finson, M. L., \& Probstein, R. F. 1968, ApJ, 154, 327

Flynn, G. J., Klöck, W., \& Krompholz, R. 1999, Lunar Plan. Sci. Conf., 30, 1073

Gail, H.-P., \& Sedlmayr, E. 1999, A\&A, 347, 594

Ghabezloo, S. 2010, Constr. Build. Mater, 24, 1796

Ghabezloo, S., \& Sulem, J. 2009, Rock Mech. Rock Eng., 42, 1

Green, D. W. E., ed. 2011, CBET 2930

Green, D. W. E., ed. 2012a, CBET 2967

Green, D. W. E., ed. 2012b, http://www.icq.eps.harvard.edu/ CometMags.html

Gundlach, B., Blum, J., Skorov, Yu. V., \& Keller, H. U. 2012, arXiv:1203.1808 1 [astro-ph.EP]

Harmon, J. K., Nolan, M. C., Howell, E. S., Giorgini, J. D., \& Taylor, P. A. 2011, ApJ, 734, L2

Hasegawa, I. 1980, Vistas Astron., 24, 59

Hasegawa, I., \& Nakano, S. 2001, PASJ, 53, 931

Haynes, W. M., ed. 2011, CRC Handbook of Chemistry and Physics, 92nd ed., CRC Press, Boca Raton, FL, pp. 6-92 and $15-42$

Hiraga, T., Anderson, I. M., \& Kohlstedt, D. L. 2004, Nature, 427,699

Ho, P.-Y. 1962, Vistas Astron., 5, 127

Howard, R. A., Moses, J. D., Vourlidas, A., Newmark, J. S., Socker, D. G., Plunkett, S. P., Korendyke, C. M., Cook, J. W., Hurley, A., Davila, J. M., Thompson, W. T., St Cyr, O. C., Mentzell, E., Mehalick, K., Lemen, J. R., Wuelser, J. P., Duncan, D. W., Tarbell, T. D., Wolfson, C. J., Moore, A., Harrison, R. A., Waltham, N. R., Lang, J., Davis, C. J., Eyles, C. J., Mapson-Menard, H., Simnett, G. M., Halain, J. P., Defise, J. M., Mazy, E., Rochus, P., Mercier, R., Ravet, M. F., Delmotte, F., Auchere, F., Delaboudiniere, J. P., Bothmer, V., Deutsch, W., Wang, D., Rich, N., Cooper, S., Stephens, V., Maahs, G., Baugh, R., McMullin, D., \& Carter, T. 2008, Space Sci. Rev., 136, 67

Huebner, W. F., Keady, J. J., \& Lyon, S. P. 1992, Ap\&SS, 195, 1

Hunter, O., Jr., \& Brownell, W. E. 2006, J. Amer. Ceram. Soc., 50,19

Iannini, G. M. 1966, IAUC 1948

Kama, M., Min, M., \& Dominik, C. 2009, A\&A, 506, 1199

Kimberley, J., \& Ramesh, K. T. 2011, Meteorit. Plan. Sci., 46, 1653

Kimura, H., Ishimoto, H., \& Mukai, T. 1997, A\&A, 326, 263

Kimura, H., Mann, I., Biesecker, D. A., \& Jessberger, E. K. 2002, Icarus, 159,529

Knight, M. M., A'Hearn, M. F., Biesecker, D. A., Faury, G., Hamilton, D. P., Lamy, P., \& Llebaria, A. 2010, AJ, 139, 926

Kováčik, J. 2005, J. Mater. Sci., 41, 1247

Kracht, R. 2011, http://tech.groups.yahoo.com/group/comets-ml/ message/18907

Kracht, R. 2012, personal communication

Kreutz, H. 1888, Publ. Sternw. Kiel, 3

Kreutz, H. 1891, Publ. Sternw. Kiel, 6

Kreutz, H. 1901, Astron. Abh., 1, 1

Krivov, A., Kimura, H., \& Mann, I. 1998, Icarus, 134, 311

Kronk, G. W. 1999, Cometography: A Catalog of Comets (Cambridge: Cambridge Univ. Press)

Kronk, G. W., ed. 2011, http://cometography.com/lcomets/ 2011w3.html

Lamy, P. L., Toth, I., Weaver, H. A., A'Hearn, M. F., \& Jorda, L. 2009, A\&A, 508, 1045

Lasue, J., Botet, R., Levasseur-Regourd, A. C., \& Hadamcik, E. 2009, Icarus, 203, 599 
Lasue, J., Botet, R., Levasseur-Regourd, A. C., Hadamcik, E., \& Kofman, W. 2011, Icarus, 213, 369

Lourens, J. V. B. 1966, Mon. Not. Astron. Soc. South Africa, 25, 52

Mann, I., Kimura, H., Biesecker, D. A., Tsurutani, B. T., Grün, E., McKibben, R. B., Liou, J.-C., MacQueen, R. M., Mukai, T., Guhathakurta, M., \& Lamy, P. 2004, Space Sci. Rev., 110,269

Marcus, J. N. 2007, Int. Comet Quart., 29, 39

Marsden, B. G. 1967, AJ, 72, 1170

Marsden, B. G. 1989, AJ, 98, 2306

Marsden, B. G. 2005, ARA\&A, 43, 75

McNaught, R. H. 2012, http://msowww.anu.edu.au/ rmn/ C2011W3.htm

Moretti, L., De Stefano, L., Rossi, A. M., \& Rendina, I. 2005, Appl. Phys. Lett., 86, 061107

Nagahara, H., Mysen, B. O., \& Kushiro, I. 1994, Geochim. Cosmochim. Acta, 58, 1951

Phani, K. K., \& Sanyal, D. 2005, J. Mater. Sci., 40, 5685

Pohn, H. 1965, IAUC 1937

Pritchard, M. E., \& Stevenson, D. J. 2000, in R. M. Canup \& K. Righter (eds.) Origin of the Earth and Moon, U. Arizona, Tucson, p. 179

Richardson, J. E., Melosh, H. J., Lisse, C. M., \& Carcich, B. 2007, Icarus, 190, 357

Rogers, D. 2005, Einstein's Other Theory: The Planck-Bose-Einstein Theory of Heat Capacity, Princeton U., Princeton, NJ, p. 73

Sagdeev, R. Z., Evlanov, E. N., Fomenkova, M. N., Prilutskii, D. F., \& Zubkov, B. V. 1989, Adv. Space Res., 9, No. 3, p. 263

Schmitt, B., Espinasse, S., Grim, R. J. A., Greenberg, J. M., \& Klinger, J. 1989, in J.Hunt \& T. D. Guyenne (eds.) Physics and Mechanics of Cometary Materials, ESA SP-302, ESTEC, Noordwijk, Netherlands, p. 65

Seargent, D. A. J. 2011, http://tech.groups.yahoo.com/group/ comets-ml/message/19078

Sekanina, Z. 1984, Icarus, 58, 81

Sekanina, Z. 2000, ApJ, 545, L69

Sekanina, Z. 2002a, ApJ, 566, 577

Sekanina, Z. 2002b, ApJ, 576, 1085
Sekanina, Z. 2003, ApJ, 597, 1237

Sekanina, Z. 2008, Int. Comet Quart., 30, 3

Sekanina, Z. 2009, Int. Comet Quart., 31, 99

Sekanina, Z. 2012, CBET 2967

Sekanina, Z., \& Chodas, P. W. 2004, ApJ, 607, 620

Sekanina, Z., \& Chodas, P. W. 2007, ApJ, 663, 657

Sekanina, Z., \& Chodas, P. W. 2008, ApJ, 687, 1415

Sekanina, Z., Hanner, M. S., Jessberger, E. K., \& Fomenkova, M. N. 2001, in E. Grün, B. Å. S. Gustafson, S. Dermott, \& H. Fechtig (eds.) Interplanetary Dust, Springer, Berlin, Germany, p. 95

Sitko, M. L., Lisse, C. M., Kelley, M. S., Polomski, E. F., Lynch, D. K., Russell, R. W., Kimes, R. L., Whitney, B. A., Wolff, M. J., \& Harker, D. E. 2011, AJ, 142, 80

Spahr, T. B., Williams, G. V., Nakano, S., \& Doppler, A., eds. 2011, MPC 77148

Spahr, T. B., Williams, G. V., Nakano, S., \& Doppler, A., eds. 2012, MPC 77565-77566

Suzuki, I., Anderson, O. L., \& Sumino, Y. 1983, Phys. Chem. Minerals, 10,38

Tait, S. 1992, Amer. Mineralogist, 77, 146

Thackeray, A. D. 1965, Mon. Not. Astron. Soc. South Africa, 24, 159

Thomas, P. C., Veverka, J., Belton, M. J. S., Hidy, A., A'Hearn, M. F., Farnham, T. L., Groussin, O., Li, J.-Y., McFadden, L. A., Sunshine, J., Wellnitz, D., Lisse, C., Schultz, P., Meech, K. J., \& Delamere, W. A. 2007, Icarus, 187, 4

Timoshenko, S. P., \& Goodier, J. N. 1970, Theory of Elasticity, 3rd ed., McGraw-Hill, New York, NY, p. 454

Utterback, N. G., \& Kissel, J. 1990, AJ, 100, 1315

Utterback, N. G., \& Kissel, J. 1995, Astrophys. Space Sci., 225, 327

Wang, H., \& Ramesh, K. T. 2004, Acta Materialia, 52, 355

Warren, N. 1969, JGR, 74, 713

Williams, G. V. 2011a, MPEC 2011-X36

Williams, G. V. 2011b, MPEC 2011-Y02

Williams, G. V. 2011c, MPEC 2011-Y23

Yomogida, K., \& Matsui, T. 1983, JGR, 88, 9513 